Old Dominion University

ODU Digital Commons

Electrical \& Computer Engineering Theses \&

Dissertations

Electrical \& Computer Engineering

Summer 2005

\title{
Electromagnetic Propagation Prediction Inside Aircraft Cabins
}

Genevieve Hankins

Old Dominion University

Follow this and additional works at: https://digitalcommons.odu.edu/ece_etds

Part of the Electromagnetics and Photonics Commons

\section{Recommended Citation}

Hankins, Genevieve. "Electromagnetic Propagation Prediction Inside Aircraft Cabins" (2005). Master of Science (MS), Thesis, Electrical \& Computer Engineering, Old Dominion University, DOI: 10.25777/2v0qyj45

https://digitalcommons.odu.edu/ece_etds/76

This Thesis is brought to you for free and open access by the Electrical \& Computer Engineering at ODU Digital Commons. It has been accepted for inclusion in Electrical \& Computer Engineering Theses \& Dissertations by an authorized administrator of ODU Digital Commons. For more information, please contact digitalcommons@odu.edu. 


\title{
ELECTROMAGNETIC PROPAGATION PREDICTION INSIDE
}

\section{AIRCRAFT CABINS}

\author{
by \\ Genevieve Hankins \\ B.S. Electrical Engineering, May 2004, Old Dominion University \\ A Thesis Submitted to the Faculty of \\ Old Dominion University in Partial Fulfillment of the \\ Requirement for the Degree of \\ MASTER OF SCIENCE \\ ELECTRICAL ENGINEERING \\ OLD DOMINION UNIVERSITY \\ August 2005
}

Approved by:

Dr. Linda Vahala (Director)

Dr. Vijayan Asąri (Member)

Dr. Ravindra Joshi (Member) 


\author{
ABSTRACT \\ ELECTROMAGNETIC PROPAGATION PREDICTION INSIDE AIRCRAFT CABINS \\ Genevieve Hankins \\ Old Dominion University, 2005 \\ Director: Dr. Linda Vahala
}

Electromagnetic propagation models for signal strength prediction within aircraft cabins are essential for evaluating and designing a wireless communication system to be implemented onboard aircraft. There are many commercially available software packages for predicting wireless system performance in conventional indoor environments. It is of interest to examine the available software to determine if the aircraft's electromagnetic environment (EME) can be modeled successfully without developing an aircraft specific prediction tool. EnterprisePlanner ${ }^{\circledR}$, a registered product of Wireless Valley Communications, Incorporated, was selected for the present effort. The performance of the prediction model was evaluated through a comparison with field measurements taken on the aircraft. It was concluded that the prediction model can accurately predict power propagation throughout the cabin. This prediction tool can enhance researchers' understanding of power propagation within aircraft cabins and will aid in future research. 
If there is no struggle, there is no progress.

-Frederick Douglass 


\section{ACKNOWLEDGMENTS}

I would like to sincerely thank my advisor, Dr. Linda Vahala, for all of her willingness, support and assistance throughout my years at Old Dominion University. Her encouragement has been an inspiration and I am very lucky to have had the opportunity to work with her. I would also like to extend my thanks to my mentor Dr. John Beggs from NASA Langley Research Center. His help and guidance was vital to this project.

I am deeply grateful to everyone at the NASA Langley HIRF Lab for not only funding this project but for also providing indispensable guidance and advice throughout this endeavor. I am especially thankful for the opportunity to work with such an exceptional group of engineers. I have really enjoyed my experience at NASA and it would not have been the same without them. (I will especially miss the inspiring lunch conversations.)

Above all, this thesis would not have been possible without the loving support of my parents and family. Many thanks to my parents, Doug and Patty Hankins, for supporting all of my endeavors. I am extremely grateful to my father for his help with reviewing and editing this thesis. Finally, a special thanks to Jason Moore for his patience and love throughout my academic studies. 


\section{TABLE OF CONTENTS}

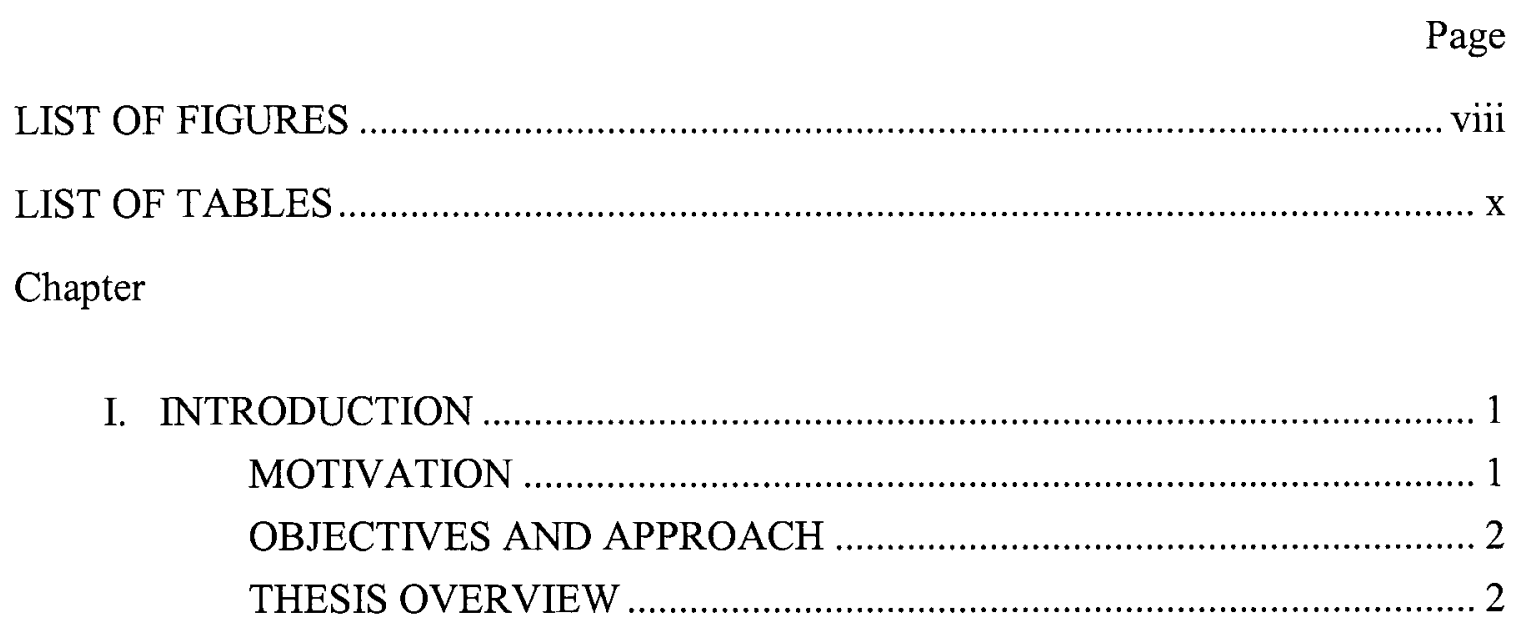

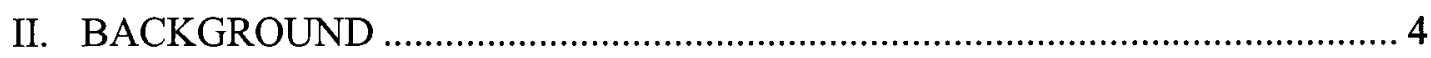

INTRODUCTION ..................................................................... 4

PREDICTION METHODS FOR ELECTROMAGNETICS ...................... 4

DERIVING THE FRIIS EQUATION .............................................. 8

ELECTROMAGNETIC ENVIRONMENT INSIDE AIRCRAFT

CAVITIES .............................................................................. 12

WIRELESS LOCAL AREA NETWORKS ......................................... 13

COVERAGE AREA ........................................................15

III. WIRELESS VALLEY SOFTWARE SUITE ............................................ 17

INTRODUCTION ..................................................................... 17

PREDICTION METHOD ............................................................. 17

CALCULATING RECEIVED POWER FROM PATH LOSS ................ 21

RECORDING AND DISPLAYING PREDICTED RESULTS................ 22

OVERVIEW OF A PREDICTION WITHIN

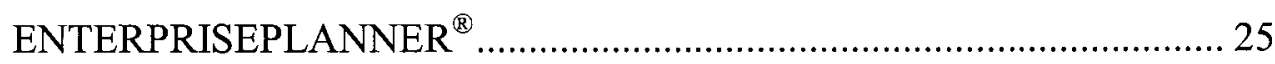

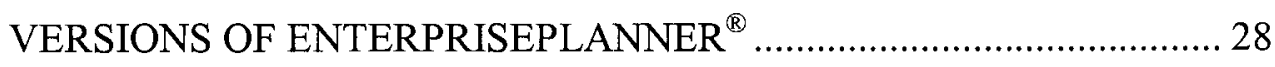

IV. EM MEASUREMENTS OF 802.11A AND 802.11B WIRELESS NETWORKS

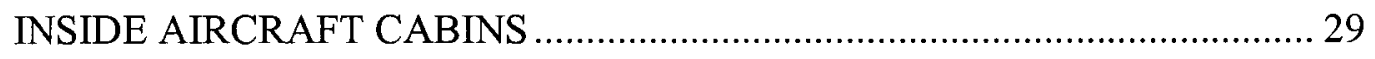

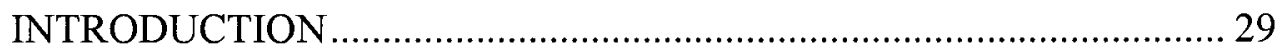

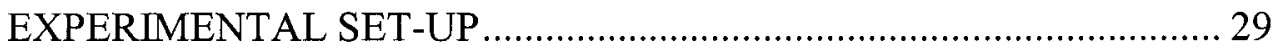




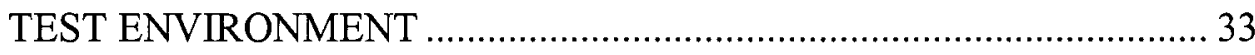

CHALLENGES ASSOCIATED WITH THE FIELD

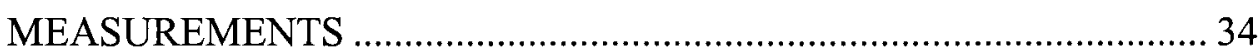

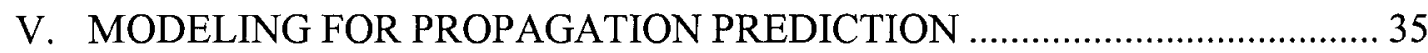

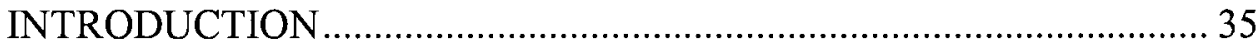

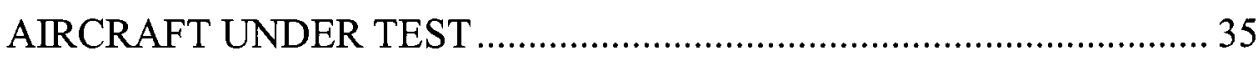

MODELING INTERIOR COMPONENTS....................................37

TUTORIAL: HOW TO CREATE A SEAT IN

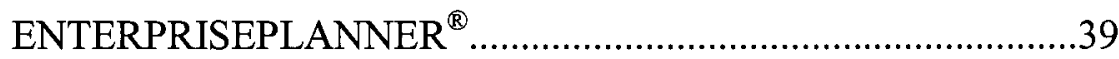

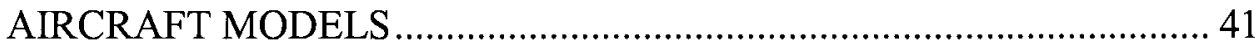

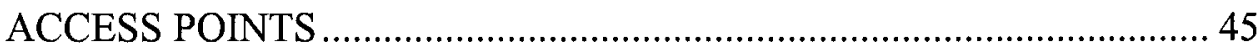

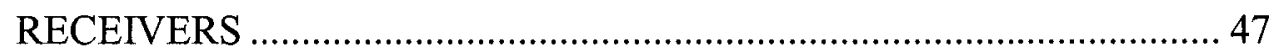

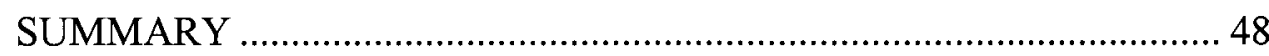

VI. PREDICTION RESULTS AND ANALYSIS …………………........................ 49

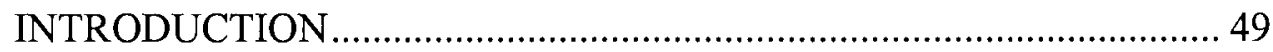

CALCULATING THE PATH LOSS EXPONENT, N............................... 49

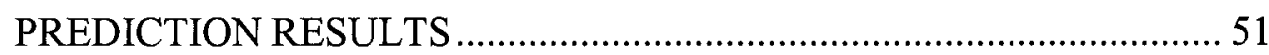

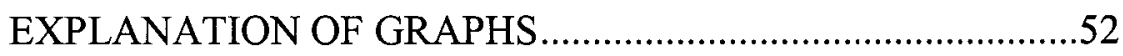

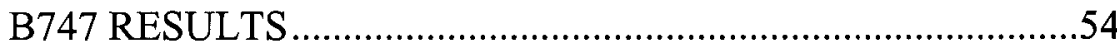

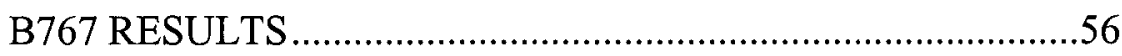

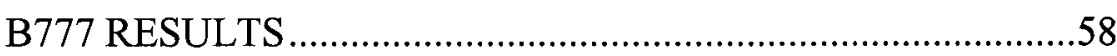

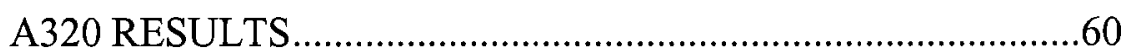

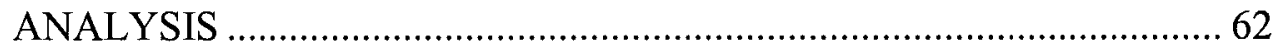

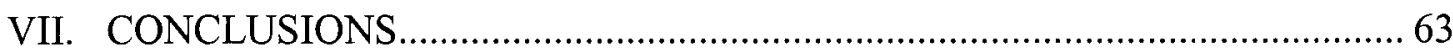

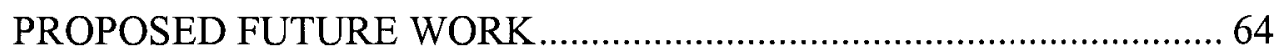

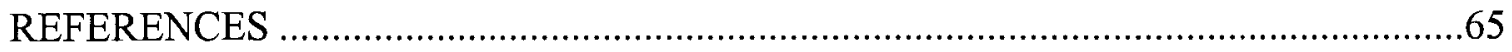

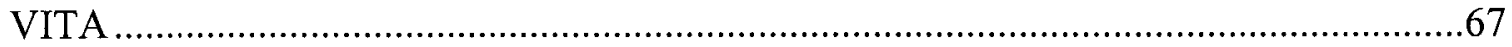




\section{LIST OF FIGURES}

Figure

Page

1: Yee Lattice. .......................................................................................................... 5

2: Transverse Electromagnetic (TEM) plane wave in free space. ................................ 9

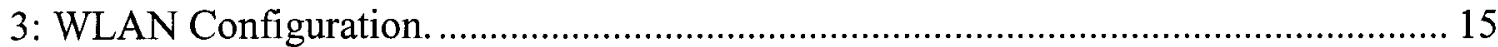

4: Ray trace of the direct path from the transmitter to the receiver. .............................. 18

5: Default values of the floor attenuation factor......................................................... 20

7: Results while in Instant Point Mode....................................................................... 22

8: Left to Right: Grid Coverage and Contour Coverage Mode results for received power

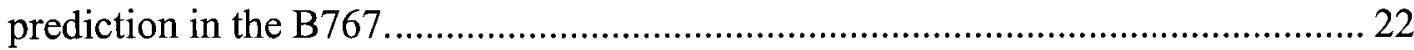

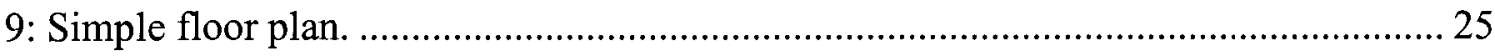

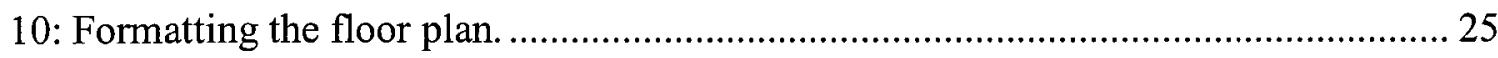

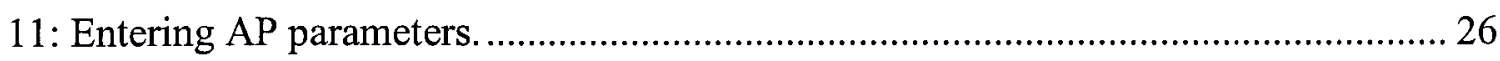

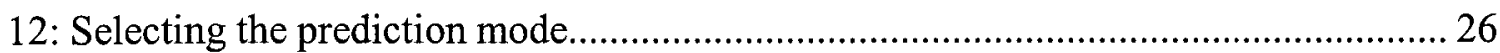

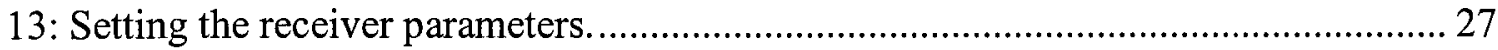

14: Selecting the path loss model and setting the path loss exponent. .......................... 27

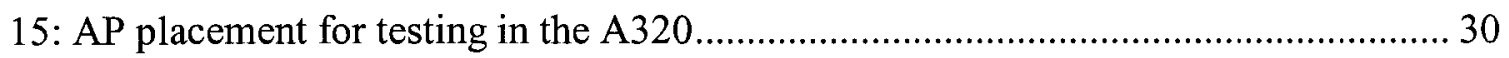

16: Mobile laptop computer running AirMagnet software for data collection................. 30

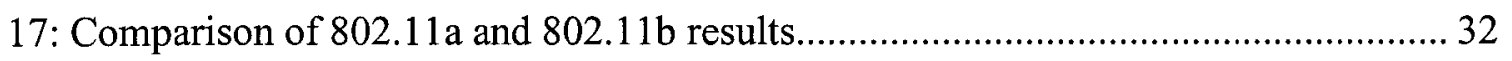

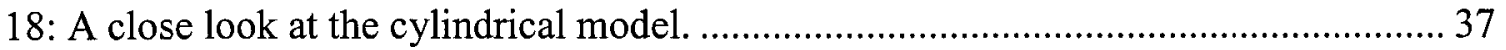

19: Dimensions of an economy class seat provided from B/E Aerospace, Inc. .............. 39

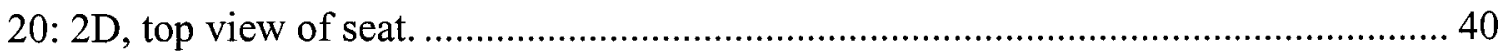

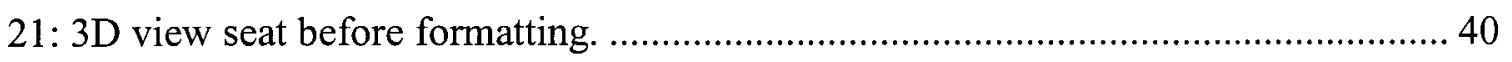

22: $3 \mathrm{D}$ view seat with one segment formatted.................................................... 41

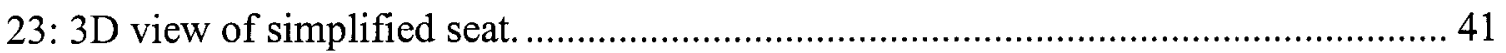

24: B767 Rectangular and Cylindrical representations featuring interior components

(3D SW view with a quarter section removed for illustration purposes) ................ 42

25: $3 \mathrm{D}$ view of $\mathrm{B} 747$ representation featuring interior components............................ 42

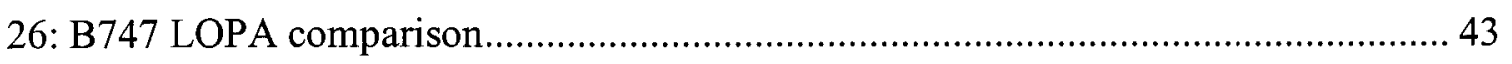

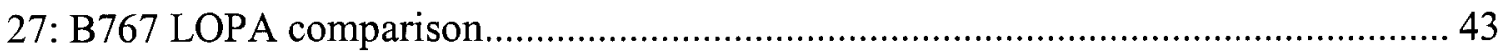

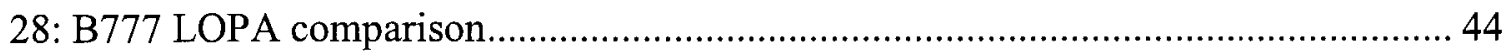

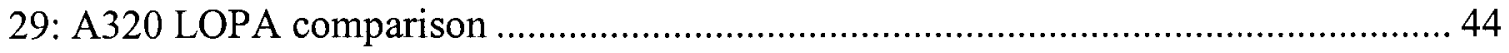

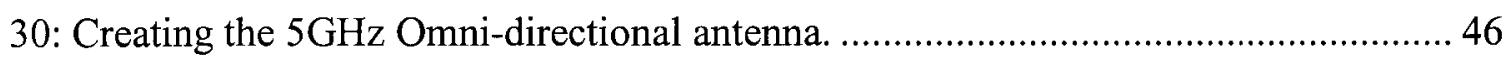


31: Calculation of path loss exponent at $5 \mathrm{GHz}$ from AirMagnet measurements for the B747. 50

32: Calculation of path loss exponent at $5 \mathrm{GHz}$ from Spectrum Analyzer measurements for the B747. 50

33: Legend for Grid Coverage results. 53

34: B747 results for 802.11 a (excluding Floor 2 of the aircraft). 54

35: B747 802.11a power propagation using Grid Coverage Mode. 54

36: B747 results for $802.11 \mathrm{~b}$ (excluding Floor 2 of the aircraft)................................. 55

37: B747 802.11b power propagation using Grid Coverage Mode................................ 55

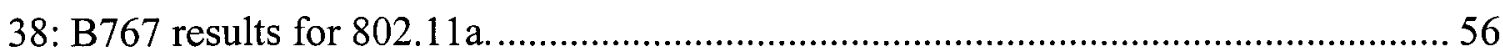

39: B767 802.11a power propagation using Grid Coverage Mode. .............................. 56

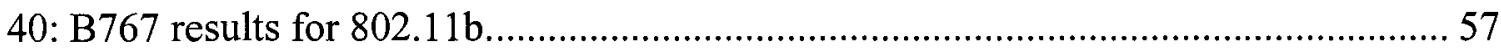

41: B767 802.11b power propagation using Grid Coverage Mode................................ 57

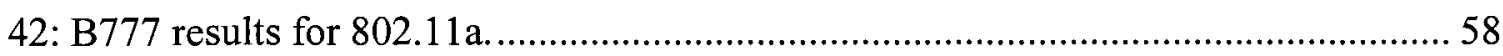

43: B777 802.11a power propagation using Grid Coverage Mode. .............................. 58

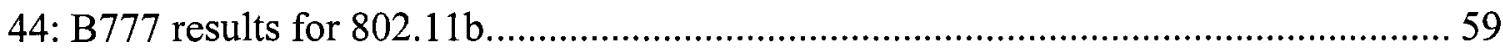

45: B777 802.11b power propagation using Grid Coverage Mode.............................. 59

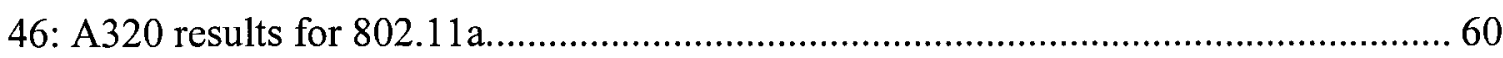

47: A320 802.11a $11 \mathrm{~b}$ power propagation using Grid Coverage Mode. ........................ 60

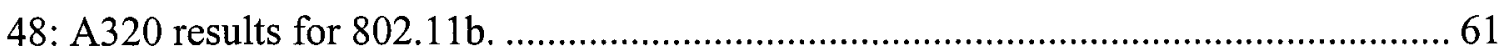

49: A320 802.11b $11 \mathrm{~b}$ power propagation using Grid Coverage Mode.......................... 61 


\section{LIST OF TABLES}

Table Page

1: Characteristics of 802.11 standards. 14

2: Descriptions of Path Loss Exponents utilized by EnterprisePlanner ${ }^{\circledR}$........................ 21

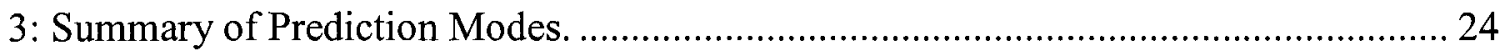

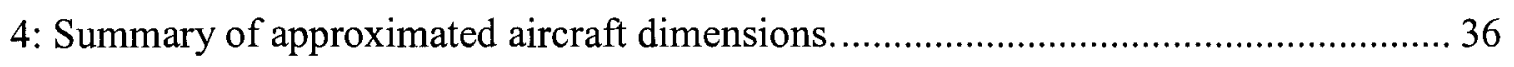

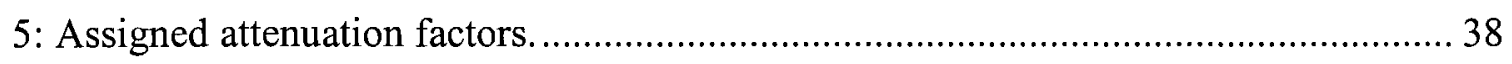

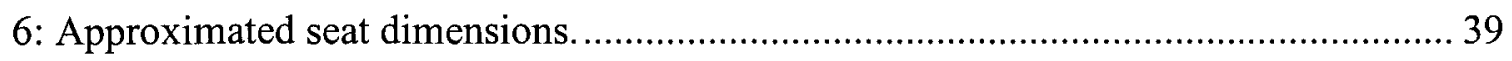

7: Summary of ORiNOCO AP2000 parameters........................................................ 45

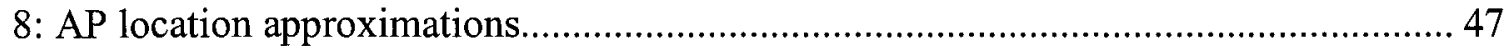

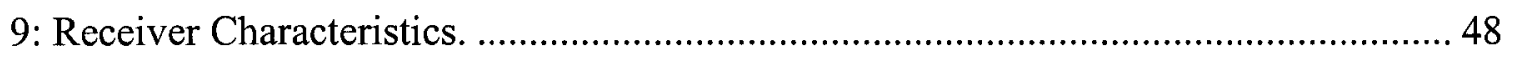

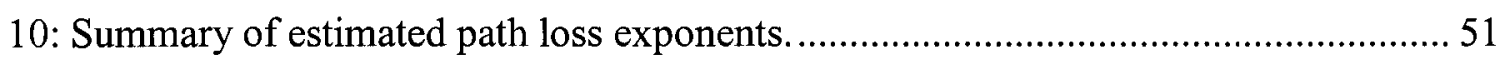

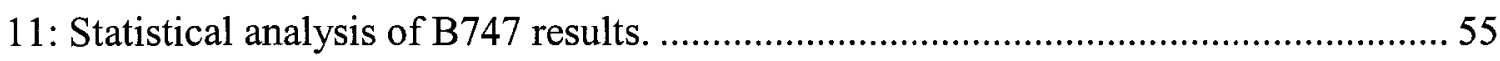

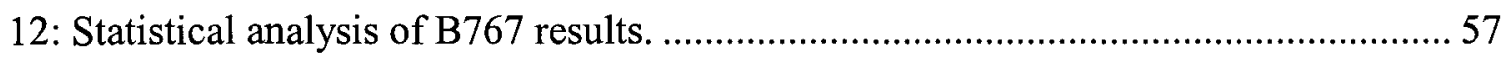

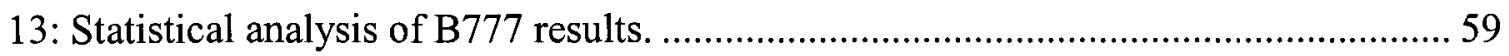

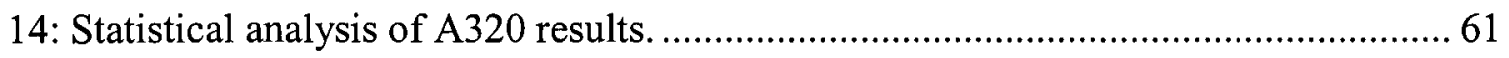




\section{CHAPTER I}

\section{INTRODUCTION}

\section{Motivation}

The wireless industry has experienced unprecedented growth over the past decade and continues to evolve at a rapid pace with the demand for additional mobile services. Advancements in the industry have led to the development of more innovative consumer applications which now cater to the needs of many different consumers, from the commercial industry to private home users [1]. In response to the traveling public's interest in wireless communications, the aviation industry is interested in increasing airborne passenger connectivity. Current plans would eventually permit cell phone usage as well as provide wireless internet access [2],[3]. In order to design these wireless systems effectively, the radio wave propagation behavior at various radio frequencies will need to be well understood. Additionally there are concerns about potential threats from electromagnetic interference (EMI) and network security [4],[5]. However before addressing these threats, it is necessary to understand how well these wireless systems would operate inside the aircraft cabin.

Although the interference risks have not been completely assessed, several airlines overseas, have already implemented wireless networks on several planes in their fleet. Airlines in the United States are expected to follow this trend after the interference risks have been assessed and they receive approval from the Federal Aviation Administration (FAA) [6],[7].

There have already been studies of wireless network performance within aircraft cabins in the United States [8]. These studies confirmed that implementing wireless networks on aircraft is quite feasible; however, they noted that further investigation into the effects and configuration of the wireless system is needed. An electromagnetic propagation prediction tool would aid in the research and design of wireless networks as well as other wireless communication systems for aircraft use. Researchers currently rely mainly on field tests, which are costly and time consuming, for insight into wave propagation in or outside the cabin. Thus, there is a desire to develop an electromagnetic 
prediction tool for aircraft applications. Such a tool would reduce the time and cost associated with extensive field tests. It could be used to plan future systems, predict potential problems or threats, and aid in combating these problems. This tool is also beneficial to commercial airlines, allowing them to design and analyze wireless networks for each plane in their fleet without interrupting their flight schedule. Additionally, a prediction tool gives airlines the opportunity to analyze several different wireless transmitters and antennas so that the optimal hardware can be chosen.

There are many commercially available software packages for designing and predicting wireless communication systems in conventional indoor environments. It is of interest to examine the available software to determine if the aircraft's electromagnetic environment (EME) can be modeled successfully without developing an aircraft specific prediction tool. EnterprisePlanner ${ }^{\circledR}$, a registered product of Wireless Valley Communications, Incorporated, was selected for the present effort. The performance of the developed model was evaluated through a comparison with test data measurements taken on several aircraft in 2003 by Embry Riddle Aeronautical University in conjunction with NASA Langley Research Center (this study is discussed in Chapter IV), [8].

\section{Objectives and Approach}

The main objective of this study is to determine whether the selected prediction tool, EnterprisePlanner ${ }^{\circledR}$, is accurate and effective for aircraft applications. This tool will be validated through a comparison with test data taken from the aforementioned study [8]. The results will be presented as a graphical comparison of received power measurements throughout the aircraft cabins. Additionally, statistical calculations such as the Mean Square Error (MAE) and the Root Mean Square Error (RMSE) will be preformed to provide further analysis of the accuracy of the prediction.

\section{Thesis Overview}

This thesis is divided into seven chapters. Chapter II provides background material on radio wave propagation, prediction methods, and wireless networking. Chapter III discusses the chosen prediction tool. Chapter IV is an overview of the experimental study, [8], whose results are used for validation. Chapter $\mathrm{V}$ discusses how 
the experimental study was modeled and Chapter VI gives the results of the predictions. A summary of the findings and overall conclusions are found in Chapter VII. Proposed future work is also presented in Chapter VII. 


\section{CHAPTER II}

\section{BACKGROUND}

\section{Introduction}

This chapter will address several topics pertaining to simulation of wireless systems. This includes suitable prediction methods and radio wave propagation. The EME in the aircraft will also be examined. Several prediction methods will be discussed and then evaluated for the current application. The theory of radio wave propagation will also be presented and its relation to the chosen prediction method will be examined. Additionally, a description of the EME inside aircraft cabins will be provided. The field measurements provided for validating EnterprisePlanner ${ }^{\circledR}$ were obtained from WLAN configurations on aircraft. Therefore the electromagnetic characteristics of wireless networks are also discussed in this chapter.

\section{Prediction Methods for Electromagnetics}

Radio waves, like other forms of electromagnetic radiation, are governed by Maxwell's equations. In realistic applications the Maxwell equations are considerably difficult to solve explicitly due to the complexity of the partial differential equations when the presence of charges, currents, complex boundary conditions and different media are considered. For modeling radio wave propagation, environments are often simplified and typically exclude the effects of additional charges or currents. Thus radio wave propagation can be solved directly using a simplified set of Maxwell equations or by considering additional approximations.

There are several techniques for directly solving Maxwell's equations; the most popular is the Finite-Difference Time-Domain method (FDTD). The FDTD method solves for both the electric and magnetic fields in time and space using a set of finite difference equations that represent the time-dependent curl equations of Maxwell's equations (developed by Yee) [9]. It is considered a class of the finite difference method, which is a standard method for solving Partial Differential Equations (PDEs). 
Equation 1 and Equation 2 are Maxwell's time-dependent curl equations, Faraday's Law and Ampere's Law, respectively, for linear, isotropic, nondispersive materials where the magnetic resistivity is taken to be zero.

$$
\begin{gathered}
\frac{\partial \bar{H}}{\partial t}=-\frac{1}{\mu} \nabla \times \bar{E} \\
\frac{\partial \bar{E}}{\partial t}=\frac{1}{\varepsilon} \nabla \times \bar{H}-\frac{\sigma}{\varepsilon} \bar{E}
\end{gathered}
$$

The FDTD method is a time-stepping method, wherein starting at time $t=0$, the electromagnetic properties are updated at each time level to give the evolving solution. In order to solve for the electric and magnetic fields throughout the entire volume, it is divided into a mesh of cubic cells. Objects are defined in the mesh by assigning material properties (permeability, $\mu$, permittivity, $\varepsilon$, and conductivity, $\sigma$ ) to each of the segments in a cubic cell. Figure 1 illustrates this mesh, commonly referred to as the Yee Lattice. As shown Figure 1, the $\mathbf{E}$ and $\mathbf{H}$ components are centered such that every $\mathbf{E}$ component is surrounded by four $\mathbf{H}$ components and every $\mathbf{H}$ component is surrounded by four $\mathbf{E}$ components.

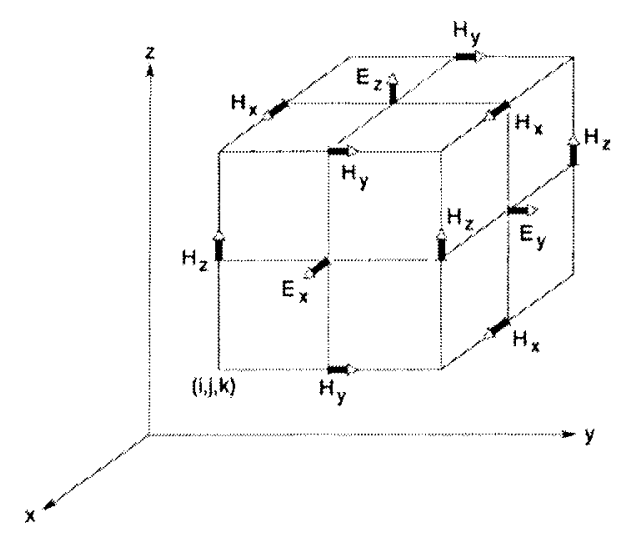

Figure 1: Yee Lattice.

This gives a simple description of how three-dimensional space is filled by an interlinked array of Faraday's Law and Ampere's Law contours. Equation 2 is used to update $\mathbf{E}$ at 
each time level $t=0 \ldots n$ and (1) is used to update $\mathbf{H}$ at each intermediate time level $t=n+1 / 2$. The system of finite-difference equations $\mathbf{E}$ and $\mathbf{H}$ can produce a new value of an electromagnetic field vector component (at any lattice point) that depends on its the previous value, the previous value of the components of the other field vectors at adjacent points and the known electric and magnetic current sources. This allows the computation of a field vector to proceed one point at a time for a given time level [9].

There are several key factors that make the FDTD method an effective and powerful prediction tool. Most importantly, the FDTD method for discretizing Maxwell's equations in space and time is relatively simple in nature (explained above). Secondly, FDTD tracks the time-varying fields throughout the entire volume which provides exceptional visualizations of the behavior of electromagnetic fields. Lastly, FDTD is a geometrically flexible method, with the exception of curved surfaces, that is capable of solving a large array of problems [10].

However, there are also limitations associated with FDTD, especially for the current application. Typical grid spacing is between $\lambda / 10$ and $\lambda / 30$ (the wavelength $\lambda=c / f$ where $c$ is the speed of light and $f$ is the frequency); for curved surfaces a more refined grid is necessary to approximate the surface with cubic cells. The FDTD method is computationally intensive and for extremely large mesh problems the computer memory and computation time required to store the propagating path on the mesh becomes impractical. Therefore for large-scale applications (thousands of wavelengths) and for modeling problems at high frequencies the FDTD method is not the ideal choice. Another concern is that the material properties needed for the simulation are usually unknown and are difficult to measure experimentally.

Another popular method of electromagnetic propagation modeling that is more suitable for high frequency and large-scale problems is ray tracing. Radio wave ray tracing utilizes a modified version of graphical ray tracing to predict the propagation of radio waves and determine field calculations. Graphical ray tracing uses the properties of geometrical optics (GO) to create a 2-D model of 3-D objects. It does this by describing each object by its geometry and light scattering properties. Graphical ray tracers use these properties to predict the exact path that light takes to a specified point. The result is a computer-generated image that resembles a photograph. Radio wave ray tracers alter the 
graphical ray tracing algorithm so that it produces propagation data rather than image data. Radio wave ray tracers use geometrical optics to trace the path of direct, reflected and transmitted fields [11]. Singly diffracted rays are also computed, but in general, GO does not account for diffracted energy. In many applications diffracted rays are computed by implementing the Geometrical Theory of Diffraction (GTD). The desired electromagnetic calculations are made by processing the raw output of the ray tracer. An interesting aspect of ray tracing is that different results can be obtained from the same ray trace by applying different techniques for processing the raw ray tracing output.

Ray tracing shares many of the same advantages as the FDTD method. It can be used to calculate field strengths across the environment to illustrate the field propagation. Like the FDTD method, it is also geometrically flexible and can account for curved surfaces in a more sufficient manner than the FDTD method. Most importantly, ray tracing is much more suitable for large-scale problems and higher frequency applications than the FDTD method. Unlike the FDTD method, the computational efficiency of ray tracing is not frequency dependent. For large-scale problems, the computation time and required computer memory will increase on account of the additional reflections and diffractions that may be encountered in a larger area. This can be avoided by limiting the number of reflections allotted in the trace; however, this may affect the accuracy of the model. Furthermore, if many reflections and diffractions are included in a large-scale problem, the ray tracing method's efficiency would still surpass that of the FDTD method. One disadvantage to the ray tracing method for the current application is that the diffraction properties of the materials in the environment are not known. Furthermore, ray tracing methods model the physical behavior of waves when they encounter electrically large objects. That is, when they encounter objects with dimensions much larger than the wavelength, $\lambda$. However, many objects in the indoor environment have dimensions within several wavelengths. Ray tracing methods will not account for the scattering effects off of these objects.

The two methods presented above are examples of deterministic electromagnetic propagation models. The results from deterministic models are not always expressed in terms of parameters that are useful for the design and analysis of wireless communications systems. Parameters such as throughput, signal-to-noise ratio (SNR), 
signal-to-interference ratio (SIR) and power density are helpful for wireless system development. Statistical models (also referred to as path loss models or a combination of analytical and empirical models) can provide EM propagation results as well as wireless system analysis. These models can provide results in seconds or minutes as opposed to the hours or days it may take deterministic models to yield results. The drawback is that path loss models do not take any of the physical phenomena (scattering, diffraction) into account when making predictions. Rather, statistical models are typically based on the principles of Friis free space transmission. The following section will discuss these principles and their relation to statistical models. The software application selected for this study uses a statistical model to calculate radio wave propagation. The details of this model will be discussed in Chapter III.

\section{Deriving the Friis Equation}

Electromagnetic waves represent a flow of energy in the direction of propagation. The power density of the electromagnetic wave is the rate at which this energy flows through a unit area of space. Power density is related to the electric and magnetic fields much like how power in electric circuits is related to voltage and current; it is the (vector) product of the two. The Poynting vector, given in (3), gives the direction of the flow of electromagnetic energy [12],[13].

$$
\bar{S}=\bar{E} \times \bar{H}
$$

The magnitude of the Poynting vector yields the instantaneous power density. However, this is often of little interest since $|\bar{S}|$ can vary over one period. Hence the average power density of one RF cycle is more commonly used in practice and is calculated similarly to the power in AC circuits. Just as in AC circuits, the average power density of a signal is given by multiplying the effective values of $\bar{E}$ and $\bar{H}$, equal to $1 / \sqrt{2}$ times the amplitudes. This yields the $1 / 2$ term in the time-averaged Poynting vector:

$$
\langle S\rangle_{T}=\frac{1}{2}(\bar{E} \times \bar{H})
$$


Equation 4 can be simplified further by excluding materials and free electric charges. The fields are assumed to be in free space where energy conservation is valid (this is a good approximation for transmission in air). In the far field or radiation zone, where $d$ is much larger than the wavelength, $\lambda$, the electric and magnetic fields are orthogonal to one another and oscillate in phase. The Poynting vector is perpendicular to both field vectors and is oriented radially away from the source; this is illustrated in Figure 2.

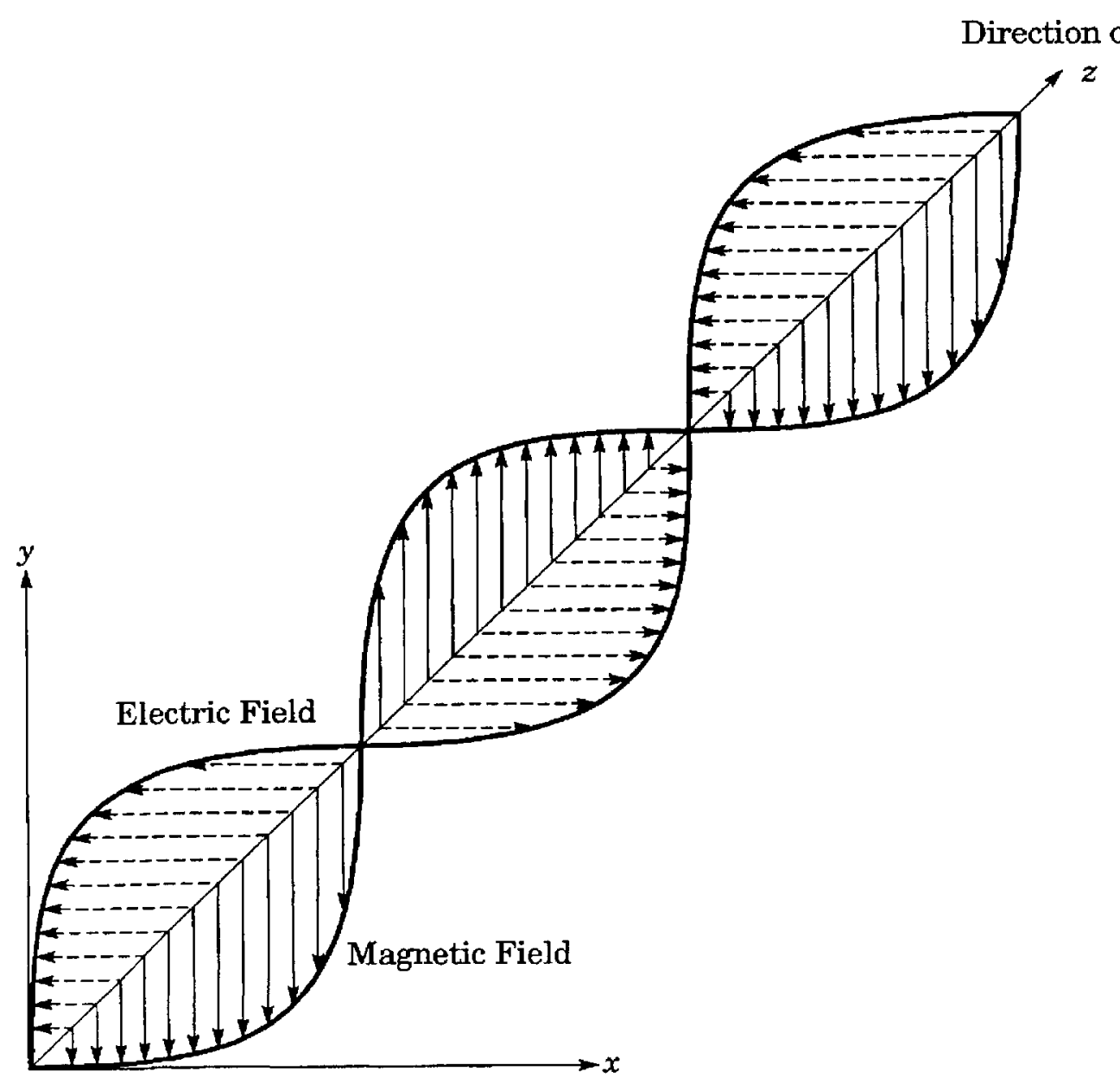

Figure 2: Transverse Electromagnetic (TEM) plane wave in free space. 
Under these conditions the fields propagate as Transverse Electro-Magnetic (TEM) waves and the ratio between the field amplitudes is constant and known as the intrinsic impedance of free space, $Z_{0}$, given in (5).

$$
\frac{\bar{E}}{\bar{H}}=Z_{0}=120 \pi \Omega \approx 377 \Omega
$$

For TEM waves, (4) can be simplified as follows using the relationship established in (5):

$$
\langle S\rangle_{T}=\frac{1}{2}(\bar{E} \times \bar{H})=\frac{\bar{E}_{e f f}^{2}}{Z_{0}}=\frac{\bar{H}_{e f f}^{2}}{Z_{0}}
$$

where $\bar{E}_{\text {eff }}$ and $\bar{H}_{\text {eff }}$ are the effective values are $\bar{E}$ and $\bar{H}$, equal to $1 / \sqrt{2}$ times the amplitudes. For isotropic radiators in free space, waves in the far-field region will be spherical; however, if a small area of spherical wave is considered in the far-field then the waves can be treated as plane waves. Therefore, to calculate the power received $P_{r}(d)$ by an antenna in the far-field region, the time-averaged power density is multiplied by the receiving area.

$$
P_{r}(d)=A_{r}\langle S\rangle_{T}
$$

The receiving area or cross section, $A_{r}$, is defined as the surface in space which captured the power of the radiated field. There is a connection between the physical size of the antenna and the receiving cross section; however, the two areas are not equal in most cases. Furthermore there is a connection between the gain of the antenna and its physical size [12]. Thus the receiving cross section can be expressed in terms of the antenna gain and wavelength, $\lambda$ as

$$
A_{r}=\frac{G \lambda^{2}}{4 \pi}
$$


For non-isotropic antennas the power density can also be written in terms of the antenna gain and the transmit power. By definition the power density is the total power radiated uniformly distributed over spherical surfaces with radius $d$ (in free space waves in farfield are spherical).

$$
\langle S\rangle_{T}(d)=\frac{P_{T} G_{T}}{4 \pi d^{2}}
$$

where $P_{T}$ is the transmit power, $G_{T}$ is the transmit antenna gain, and $4 \pi d^{2}$ is the surface area of a sphere with radius $d$. Equation 9 is equivalent to (6)

$$
\langle S\rangle_{T}=\frac{1}{2}(\bar{E} \times \bar{H})=\frac{\bar{E}_{e f f}^{2}}{Z_{0}}=\frac{\bar{H}_{e f f}{ }^{2}}{Z_{0}}=\frac{P_{T} G_{T}}{4 \pi d^{2}}
$$

Now substituting (7) yields:

$$
P_{r}(d)=A_{r}\langle S\rangle_{T}=\frac{P_{T} G_{T}}{4 \pi d^{2}} A_{r}=\frac{P_{T} G_{T}}{4 \pi d^{2}} \cdot \frac{G \lambda^{2}}{4 \pi}=\frac{P_{T} G_{T} G_{R} \lambda^{2}}{(4 \pi d)^{2}}
$$

Equation 11 is known as the Friis transmission equation or free space loss equation (in this case the system loss factor, $L$, is equal to one; this quantity is not related to propagation).This equation can be used to calculate the received power in line-of-sight (LOS) situations where there is a clear, direct path from the transmitting antenna to the receiving. However, it is often more practical to write this equation in terms of path loss. Path loss (PL) represents the signal attenuation as a positive quantity and is defined as the difference (in $\mathrm{dB}$ or $\mathrm{dBm}$ ) between the effective transmitted power and the received power. The expression for path loss may or may not include the effects of the antenna gains [25]. Equation 12 excludes the effects of antenna gain.

$$
P L(d B)=10 \log \left(\frac{P_{T}}{P_{R}}\right)=-10 \log \left(\frac{\lambda^{2}}{(4 \pi d)^{2}}\right)=20 \log \left(\frac{4 \pi d}{\lambda}\right) \quad[d B \text { or } d B m]
$$


The free space model and hence (12) are only valid in the far-field region. Furthermore, it is clear that (11) and (12) do not hold for $d=0$. For these reasons large-scale propagation models use a close-in distance, $d_{o}$, as a known received power reference point. Therefore the received power, $P_{R}(d)$, for $d>d_{o}$ can be expressed in terms of the reference distance signal power.

$$
P_{R}(d)=P_{R}\left(d_{0}\right)\left(\frac{d_{0}}{d}\right)^{2}
$$

Substituting (13) into (12) yields the following expression for average path loss.

$$
\overline{P L}(d)=P L\left(d_{0}\right)+10 n \log \left(\frac{d}{d_{0}}\right)
$$

where $n$ is the path loss exponent. For free space, $n=2$, as illustrated in (13). This free space model is accurate for predicting signal strength in LOS situations and can be used to estimate signal strength under non-line-of-sight (NLOS) conditions. Obviously for NLOS calculations this method ignores all obstructions. For LOS and NLOS calculations, reflections, diffractions and other physical affects are ignored. However, many statistical methods attempt to compensate for this error. There are a variety of methods for which the effects of obstructions can be approximated. In many techniques the path loss exponent is approximated from experimental data and substituted into Equation (14) to yield more accurate results. Another approach, which is incorporated in EnterprisePlanner ${ }^{\circledR}$, estimates the losses encountered from passing through obstructions and adds these losses to the free space path loss. This will be discussed further in Chapter III. Although approximations are made with path loss models and that reflected, diffracted and scattered waves are ignored, these models have shown to be accurate for predicting EM propagation in indoor environments [14],[15,[16].

\section{Electromagnetic Environment inside Aircraft Cavities}

The shape, material, and conditions within aircraft cabins create a complex environment for electromagnetic propagation. Complex cavities are loosely characterized 
as a non-regular geometry of conducting surfaces. The cylindrical shape of the cabin combined with the outer metallic, aluminum alloy shell creates an environment in which the interior fields are not plane waves. The conducting materials on the exterior of the aircraft can cause multiple reflections and some internal components (i.e. overhead bins) of the aircraft have been found to be just as reflective as metals at high frequencies [8]. Other internal components such as seats, lavatories, and galleys whose dimensions vary from two to fifteen wavelengths (at $2.4-5 \mathrm{GHz}$ for wireless communication systems) will exhibit complex scattering behavior depending on their shape and material properties [17]. This can cause waves to be reflected, absorbed and/or scattered. Small objects (« $\lambda)$ have weak scattering effects, characterized by Rayleigh Scattering and for practical purposes their effects can be ignored [17],[18]. Additionally, the passengers could potentially reflect and/or absorb radio waves.

Considering all of these conditions the aircraft cavity is quite complex. As discussed above, the FDTD and ray tracing methods would account for much of the physical interaction of radio waves in this environment but at the cost of computational complexity. That is why the simplified methods of path loss models are of particular interest. The goal of this work was to determine if EnterprisePlanner ${ }^{\circledR}$, which uses path loss models, can provide accurate results for aircraft modeling applications and thus provide a more efficient method for designing, analyzing and planning wireless communication systems. Furthermore, path loss models are capable of providing results more applicable to wireless communication system analysis such as received power, throughput, SNR, and SIR.

\section{Wireless Local Area Networks}

The focus of this thesis is on radio wave propagation and prediction within aircraft cabins; therefore, some specific aspects of wireless networking will not be addressed. This section will discuss the radio wave aspects of the wireless system and its basic functionality.

Wireless Local Area Networks (WLANs) use electromagnetic waves to relay information from one user to another without relying on any physical connections. An Access Point (AP) transmits (or receives) data signals to receiving (or transmitting) 
laptop/PC client cards creating the wireless network. Since WLAN hardware is relatively low in cost and requires minimal retrofitting, it is of particular interest to the commercial aviation industry. Comparatively, a wired LAN would involve considerable retrofitting which may not be feasible due to cost, weight and the time required to take the aircraft out of service for installation.

WLANs are governed by several different IEEE 802.11 standards. The current leading products comply with the $802.11 \mathrm{a}, 802.11 \mathrm{~b}$, and $802.11 \mathrm{~g}$ standards. Each standard specifies the operational frequency, signal modulation, and data transfer rate. The Table 1 below outlines these characteristics.

Table 1: Characteristics of 802.11 standards.

\begin{tabular}{|c|c|c|l|}
\hline $\begin{array}{c}\text { Wireless } \\
\text { Standard }\end{array}$ & Frequency & $\begin{array}{c}\text { Nominal Data } \\
\text { Transfer Rate }\end{array}$ & \multicolumn{1}{|c|}{ Signal Modulation } \\
\hline $802.11 \mathrm{a}$ & $\begin{array}{c}5 \mathrm{GHz} \\
\text { UN-II Band }\end{array}$ & $54 \mathrm{Mbps}$ & $\begin{array}{l}\text { Coded Orthogonal Frequency } \\
\text { Division Multiplexing (COFDM) }\end{array}$ \\
\hline $802.11 \mathrm{~b}$ & $\begin{array}{c}2.4 \mathrm{GHz} \\
\text { ISM Band }\end{array}$ & $11 \mathrm{Mbps}$ & $\begin{array}{l}\text { Direct Sequence Spread } \\
\text { Spectrum (DSSS) }\end{array}$ \\
\hline $802.11 \mathrm{~g}$ & $\begin{array}{c}2.4 \mathrm{GHz} \\
\text { ISM Band }\end{array}$ & $54 \mathrm{Mbps}$ & $\begin{array}{l}\text { Coded Orthogonal Frequency } \\
\text { Division Multiplexing (COFDM) }\end{array}$ \\
\hline
\end{tabular}

The $802.11 \mathrm{a}$ and b standards were released simultaneously in 1999. Later, IEEE 802.11g was created as a hybrid of the 802.11a and 802.11b standards, offering the higher data transfer rates of $802.11 \mathrm{a}$ at the operational frequency of $802.11 \mathrm{~b}$. As illustrated in Table 1 the standards differ by frequency and signal modulation; signal modulation ultimately determines the data transfer rate. WLANs which comply with the 802.11a standard operate at a higher frequency, in the Unlicensed National Information Infrastructure (UN-II) band, which is a relatively unpopulated frequency band. WLANs complying with $802.11 \mathrm{~b}$ and $802.11 \mathrm{~g}$ standards, however, operate in the unlicensed Industrial, Scientific, and Medical (ISM) band making them more susceptible to interference from other devices such as microwaves, or cordless telephones operating in the same frequency. Both 802.11a and 802.11g standards offer higher data transfer rates than the $802.11 \mathrm{~b}$ standards by utilizing the same signal modulation technique, COFDM. COFDM modulation is a technique for transmitting large amounts of data over a radio wave and provides higher data transfer rates than other modulation schemes. COFDM 
splits a data signal into multiple sub-signals then transmits the sub-signals at several different carrier frequencies (all of which are Orthogonal to one another) simultaneously. This type of modulation is considered a multi-carrier modulation since it transmits using more than one frequency simultaneously. Multi-carrier modulation allows the signal to transmit with the same amount of bandwidth as single-carrier modulation but carry much more information. Thus, COFDM modulation offers high transfer rates without sacrificing bandwidth. The $802.1 \mathrm{lb}$ standard uses a different type of modulation scheme which does not allow for such high data transfer in the ISM band. DSSS modulation is a single-carrier modulation technique that spreads a data signal (over a fixed bandwidth) while reducing the signals power density. This produces a modulated signal that resembles low-level noise dispersed around a single frequency; therefore, DSSS modulation is a single-carrier modulation scheme [19].

\section{Coverage Area}

The coverage area of an AP or cell in the WLAN is by definition the region or area where users will receive adequate radio signal strength for communication. In indoor and aircraft applications obstructions will limit the coverage area of an AP therefore several APs are typically used to establish the WLAN. The coverage area and the use of additional APs are illustrated in Figure 3. The circular, dashed lines in the figure represent the coverage area of an AP.

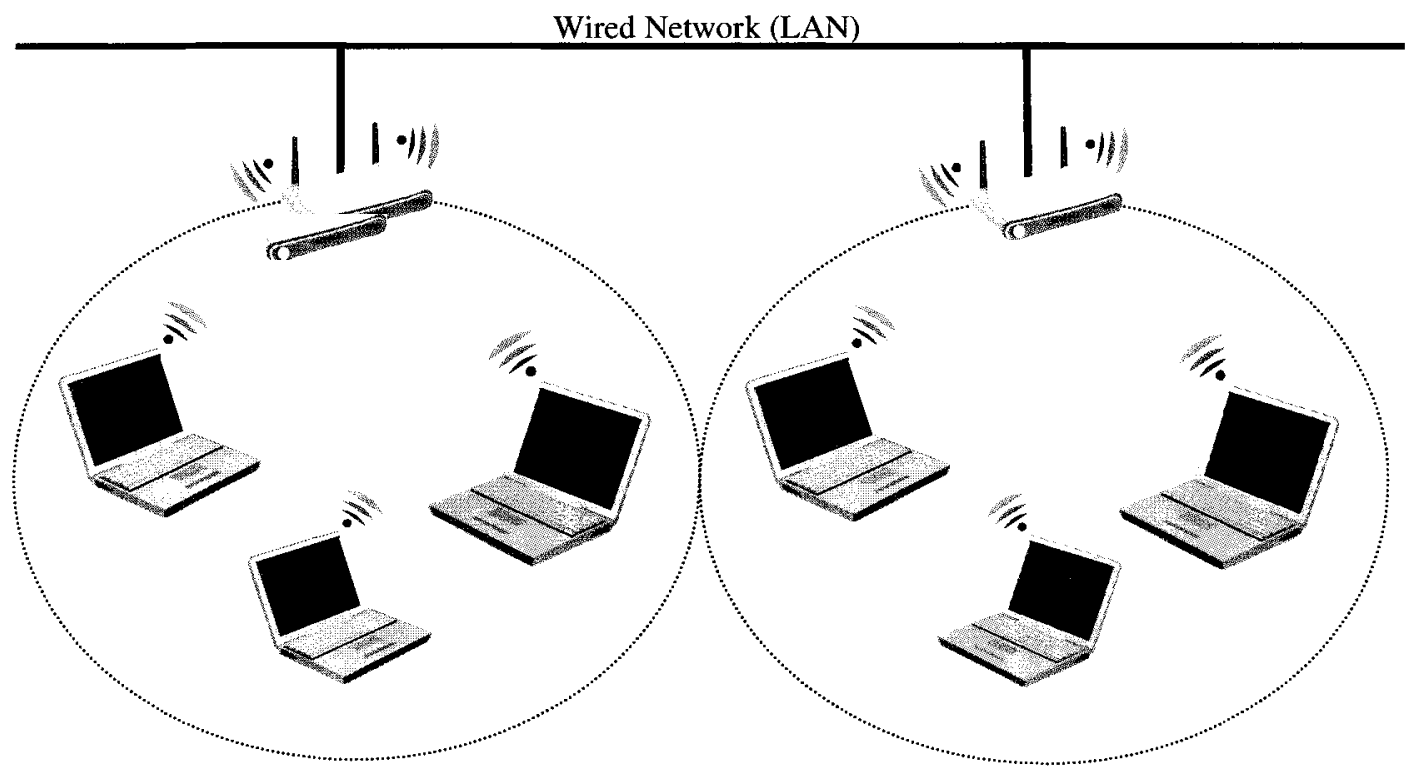

Figure 3: WLAN Configuration. 
Typically WLANs operating at $2.4 \mathrm{GHz}$ have a larger coverage area than those operating at $5 \mathrm{GHz}$. This can be attributed to the frequency effects on propagation as shown in Equation (11), smaller wavelengths will cause the signal strength to decay more rapidly (assuming signals have the same transmit power). Furthermore, the signal's wavelength also determines how the signal will interact with objects in the environment. As discussed previously, objects that are electrically large will affect the signal by causing reflections, diffractions, and absorptions. However many objects in indoor applications have dimensions within several wavelengths which causes more complex behavior. Therefore signals with smaller wavelengths (in this case $5 \mathrm{GHz}$ signals) will encounter more objects that will be considered electrically large. In the same environment, signals with larger wavelengths (e.g. $2.4 \mathrm{GHz}$ ) may not perceive the same object as electrically large. Therefore signals at $2.4 \mathrm{GHz}$ are more likely to experience less detrimental affects such as scattering, based on the objects shape and material characteristics. In this respect, objects that would be absorbed or reflected by a $5 \mathrm{GHz}$ signal may not necessarily be absorbed or reflected by a $2.4 \mathrm{GHz}$ signal. Reflected, diffracted and attenuated pieces of a signal reach the receiver at different times and have traveled a longer distance than a signal received directly at the receiver. This is called multipath. Multipath signals are basically delayed copies of the transmitted signal that contain less energy (have smaller amplitudes). All signals recombine at the receiver, the effects of multipath distort the received signal. This causes errors when the signal is demodulated. COFDM modulation is less susceptible to these errors than DSSS modulation. At $5 \mathrm{GHz}$ the likelihood of multipath increases; however, 802.11a uses COFDM modulation which makes is less susceptible to multipath inference. On the other hand at $2.4 \mathrm{GHz}$ there is comparatively less multipath but $802.11 \mathrm{~b}$ systems are usually more susceptible to multipath effects because of their modulation scheme. Additionally considering these conditions, 802.11g systems are the least susceptible to multipath interference.

Studies [20], [21], [22] and [23] have shown that in indoor environments $802.11 \mathrm{a}$ WLANs do not have the dynamic range of 802.11 b WLANs. However, at $5 \mathrm{GHz} 802.11 \mathrm{a}$ provides higher data transfer rates. Therefore a weak signal (low received power) will provide a much higher data transfer rate than a weak $802.11 \mathrm{~b}$ signal. Thus in this respect the coverage range of $802.11 \mathrm{a}$ and $802.11 \mathrm{~b}$ are quite comparable. 


\section{CHAPTER III}

\section{WIRELESS VALLEY SOFTWARE SUITE}

\section{Introduction}

EnterprisePlanner ${ }^{\mathbb{B}}$ is a software application used for engineering, managing, and planning wireless communication networks for indoor office type environments. It is powered by Autodesk technology, a tool used widely in the engineering field for creating 3D models. This allows 3D models to be created directly within the application or to be easily imported from other applications. EnterprisePlanner ${ }^{\circledR}$ combines the professional drawing capabilities of AutoCAD with powerful propagation prediction abilities, making it a very versatile prediction tool [24].

This tool was developed to assist in designing various indoor wireless systems. Although this tool was intended for simulating results inside buildings, its prediction methods make it a likely candidate for predicting aircraft environments. This is also supported by more recent work onboard aircraft [16]. It was reported in [16] that for larger, two aisle, long-haul aircraft the propagation characteristics are similar to that of an indoor office environment.

\section{Prediction Method}

Chapter II discussed several methods for modeling electromagnetic propagation. EnterprisePlanner ${ }^{\circledR}$ utilizes a method related to the free space path loss model. For practical purposes Equation (14) is presented again. Recall that $n$ is the path loss exponent. As mentioned in the previous chapter, free space models can be used as a basis for predicting actual power propagation. To increase the accuracy of these models several techniques could be used to account for the additional losses in the environment.

$$
P L(d)=P L\left(d_{0}\right)+10 n \log \left(\frac{d}{d_{0}}\right)
$$

EnterprisePlanner ${ }^{\circledR}$ allows the user to choose between two techniques for modeling these additional losses. The user can also choose to use the Distance Dependent Path Loss 
Model which is equivalent to (14). In this model, no obstructions are considered for determining the path loss (even if obstructions are modeled) and the results are based solely on the T-R separation distance, $d$ and the transmitter and receiver characteristics. The distance $d$ is found by incorporating a simplified ray tracing technique. EnterprisePlanner ${ }^{\circledR}$ uses a single ray trace to trace the direct path from the transmitter to the receiver where all obstructions are ignored. This is illustrated in Figure 4.

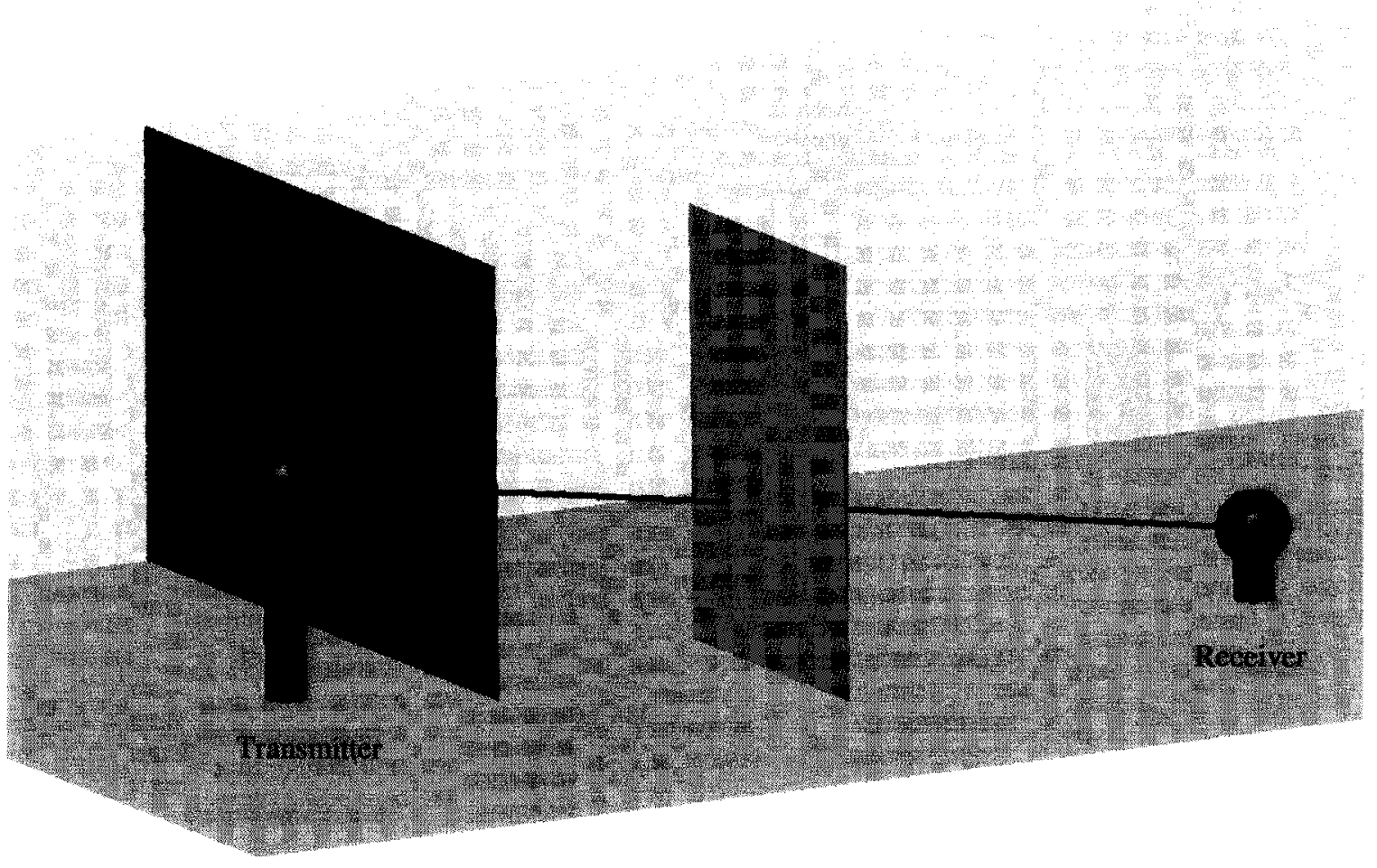

Figure 4: Ray trace of the direct path from the transmitter to the receiver.

In addition to providing the T-R separation distance, the direct ray path also determines the number and type of partitions intersected, the time-of-arrival, direction-of-arrival and the direction-of-departure. These are used to calculate the gains of the transmitting and receiving antennas [24].

Another model within EnterprisePlanner ${ }^{\circledR}$ which includes the effects of obstructions is the Wall/Floor Attenuation Factor model. This model is described by (15) when the transmitter and receiver are on the same floor. 


$$
P L(d)=P L\left(d_{0}\right)+10 n \log \left(\frac{d}{d_{0}}\right)+\sum_{i}\left[\left(P_{i}\right)\left(A F_{i}\right)\right]
$$

where $P_{i}$ is the number of partitions of type $i$, having an attenuation factor of $A F_{i}$. The attenuation factor is the path loss (in $\mathrm{dB}$ ) sustained from propagating through the partition and is of course frequency dependent. EnterprisePlanner ${ }^{\circledR}$ contains a database of attenuation factors at several frequencies for common building materials. The user can select the building material that best describes a partition or assign a custom attenuation factor based on measurement data. There has been extensive research on the path loss encountered from various materials and researchers have developed a large database of attenuation factors. Additional attenuation factors can found in [25]. In (15) the total of the loss sustained from all partitions along the direct path to the receiver is represented by summing all of the different material losses [24].

For cases where the transmitter and receiver are on different floors this model uses (16) for calculating path loss.

$$
P L(d)=P L\left(d_{0}\right)+10 n \log \left(\frac{d}{d_{0}}\right)+F A F \quad[d B]
$$

$F A F$ is the floor attenuation factor, and the default settings are shown in Figure 5. In this event path loss from partitions are not included. The Wall/Floor Attenuation Factor model chooses between the models in (15) and (16) based on whether or not the ray from the transmitter to the receiver passes through a ceiling. Ceilings are typically specified by adding ceiling tiles to the drawing. If ceiling tiles have been added to a drawing, this model will use (16) in the event that the ray passes through the ceiling tiles. Otherwise this model will revert to (15) regardless of whether the receiver is on a different floor. On the other hand, if ceiling tiles have not been specified at any location, this model automatically uses the model in (16) if the ray travels to another floor [24]. 


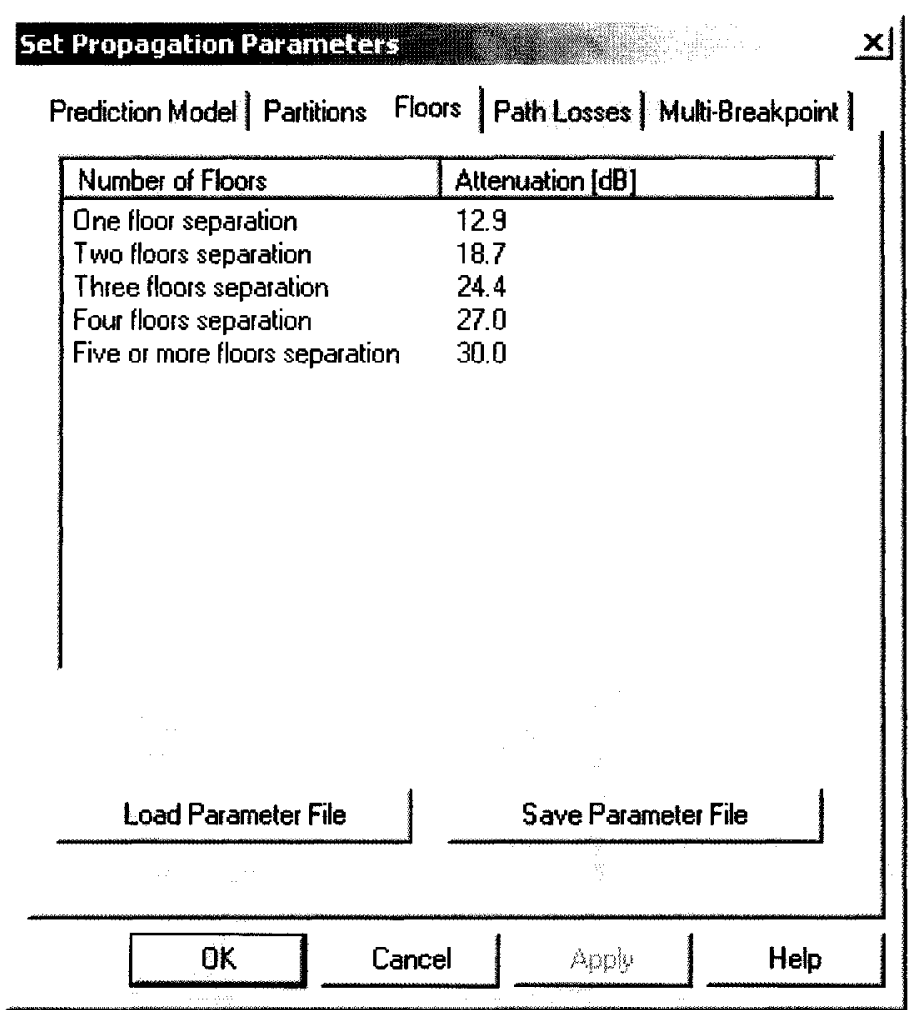

Figure 5: Default values of the floor attenuation factor.

The True Point-to-Point Models account for all partitions intersected along the path from the transmitter to receiver regardless of their locations. If the ray passes through a ceiling (please note the ceiling discrepancies described above), the FAF along with the path loss from any obstructions are considered. The expression for calculating path loss with this model effectively combines (15) and (16) making these models the most accurate [24].

$$
P L(d)=P L\left(d_{0}\right)+10 n \log \left(\frac{d}{d_{0}}\right)+\sum_{i}\left[\left(P_{i}\right)\left(A F_{i}\right)\right]+F A F
$$

In addition to the three offered models for path loss, EnterprisePlanner ${ }^{\circledR}$ allows three different path loss exponents to be specified. This allows for additionally flexibility within each of the models. Table 2 outlines the characteristics of each path loss exponents. The default value for each type of exponent is $n=2$; however, all values can be specified by the user (this includes the distances in the Multi-Breakpoint exponent). 
Table 2: Descriptions of Path Loss Exponents utilized by EnterprisePlanner ${ }^{\circledR}$.

\begin{tabular}{|c|c|c|}
\hline Type of Exponent & Calculation & Description \\
\hline $\begin{array}{l}\text { Single Path Loss } \\
\text { Exponent }\end{array}$ & $n=C$ & $\begin{array}{l}\text { Uses the same value of } \\
n \text { for all calculations }\end{array}$ \\
\hline $\begin{array}{l}\text { Multiple Path Loss } \\
\text { Exponent }\end{array}$ & $\begin{array}{cc}C & \text { same floor } \\
C_{1} & \text { 1 floor separation } \\
C_{2} & 2 \text { floor separation } \\
\vdots & \vdots \\
C_{\max } & \max \text { floor separation }\end{array}$ & $\begin{array}{l}\text { Uses a different } n \\
\text { depending on the } \\
\text { number of floor } \\
\text { separations }\end{array}$ \\
\hline $\begin{array}{c}\text { Multi-Breakpoint } \\
\text { Path Loss } \\
\text { Exponent }\end{array}$ & $\begin{array}{cc}C & d<c \\
C_{1} & c \leq d \leq c_{1} \\
n=C_{2} & c_{1} \leq d \leq c_{2} \\
\vdots & \vdots \\
C_{x} & c_{x-1} \leq d \leq c_{x}\end{array}$ & $\begin{array}{l}\text { Uses a different } n \\
\text { based on the T-R } \\
\text { distance }\end{array}$ \\
\hline
\end{tabular}

\section{Calculating Received Power from Path Loss}

The results from the path loss calculations are combined with the transmitter and receiver properties to determine the predicted received power. From the definition of path loss in (12) the received power in terms of path loss is given by

$$
P_{r}(d)=P_{T}-P L(d) \quad[d B]
$$

where all values are in units of $\mathrm{dBm}$. Equation 18 assumes that the antennas are isotropic, in terms of a practical antenna, (18) becomes

$$
P_{r}(d)=P_{T}+G_{T}+G_{R}-P L(d) \quad[d B]
$$

where $G_{T}$ and $G_{R}$ are the transmitter and receiver antenna gains. 


\section{Recording and Displaying Predicted Results}

Results are obtained for the selected Path Loss models and path loss exponent through various prediction modes. Many of the modes ultimately give the same results but they are displayed in several different ways. Other modes in EnterprisePlanner ${ }^{\circledR}$ are used specifically for improving network performance or coverage area. Figure 7 and Figure 8 are examples of results from three different modes. Table 3 lists and summarizes the various prediction modes.

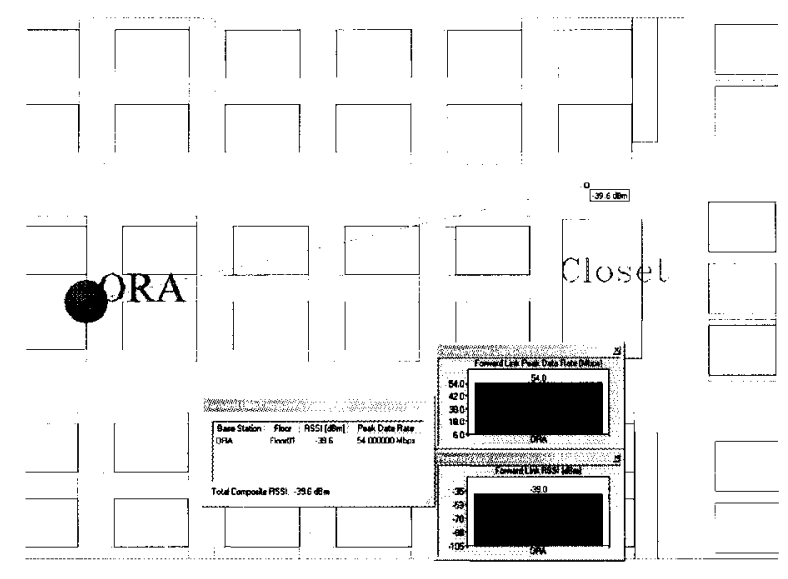

Figure 7: Results while in Instant Point Mode.

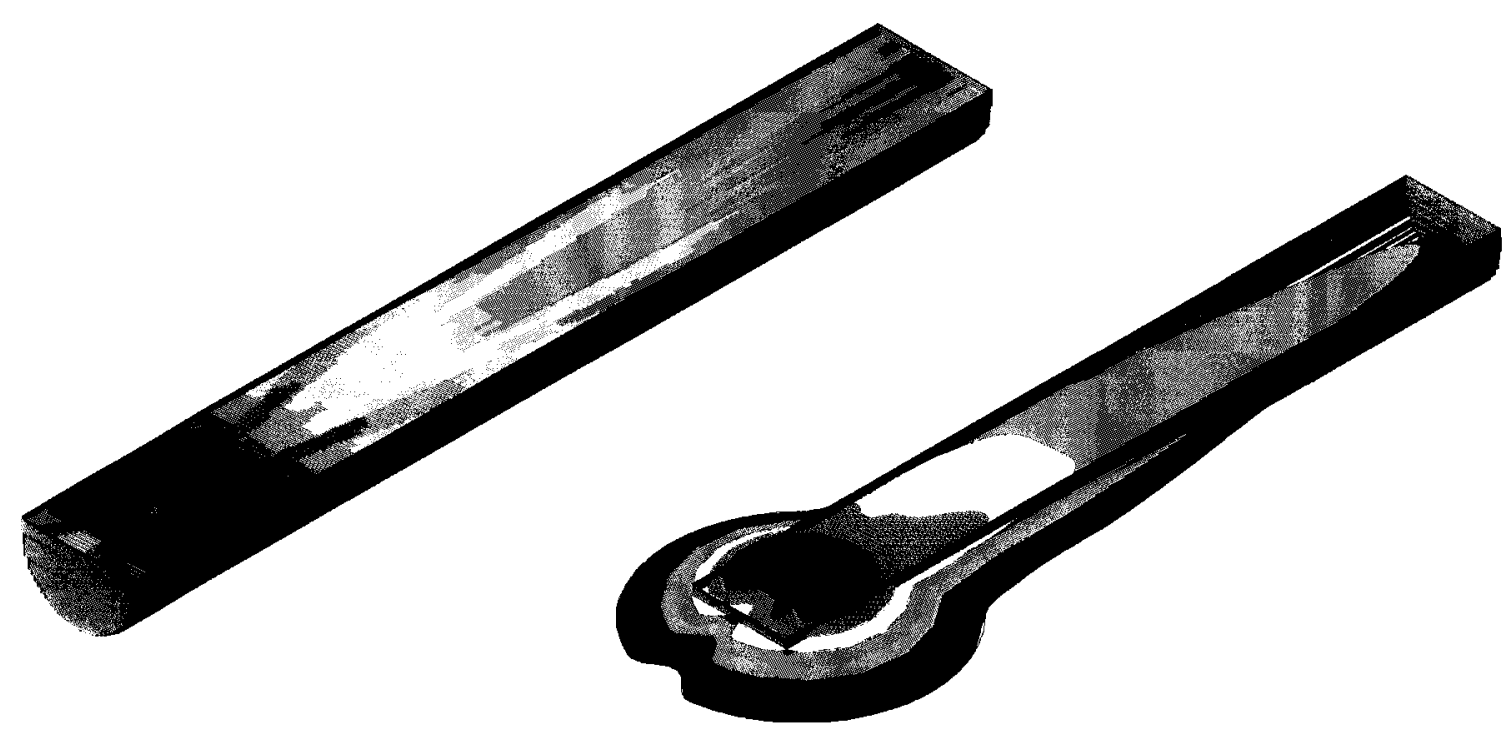

Figure 8: Left to Right: Grid Coverage and Contour Coverage Mode results for received power prediction in the $\mathbf{B 7 6 7 .}$ 
Although all of these modes are useful for designing and analyzing wireless systems, the Instant Point Mode and Grid Coverage Mode are of particular interest at this stage of research. The antenna modes will be beneficial for planning the wireless networks for implementation. The Grid Coverage Mode displays the power measurements as a color grid across the test area. This gives an illustration of how the power propagates through part or all of the test area. However, the measured data results were obtained at specific locations and the power at these locations is not easily obtained from this mode. The Instant Point Mode was used to obtain the values needed to compare the measured data directly. 
Table 3: Summary of Prediction Modes.

\begin{tabular}{|c|c|}
\hline Prediction Mode & Description \\
\hline $\begin{array}{c}\text { Antenna Positioning } \\
\text { Mode }\end{array}$ & $\begin{array}{l}\text { Assists in determining the ideal locations for transmitting } \\
\text { antennas. The antenna is moved throughout the drawing and } \\
\text { specific points of interest are monitored to find the optimal } \\
\text { location of the antenna. }\end{array}$ \\
\hline $\begin{array}{c}\text { Antenna Reorientation } \\
\text { Mode }\end{array}$ & $\begin{array}{l}\text { Assists in determining the ideal position for transmitting } \\
\text { antennas just as the Antenna Positioning mode. The } \\
\text { difference is that the antenna's orientation changes, not its } \\
\text { position. This allows the user to select the optimal } \\
\text { orientation. }\end{array}$ \\
\hline Instant Point Mode & $\begin{array}{l}\text { The cursor becomes a mobile receiver and can be moved } \\
\text { throughout the drawing to record data measurements. } \\
\text { Provides power, SIR, SNR, relative power, power density } \\
\text { and throughput measurements. }\end{array}$ \\
\hline $\begin{array}{c}\text { Contour Coverage } \\
\text { Mode }\end{array}$ & $\begin{array}{l}\text { Displays equal power closed contours around each radiating } \\
\text { source in a system. The user specifies power levels of } \\
\text { interest. The mode is also capable of displaying equal SIR, } \\
\text { SNR, relative power, power density and throughput closed } \\
\text { contours. }\end{array}$ \\
\hline $\begin{array}{l}\text { 3D Contour Coverage } \\
\text { Mode }\end{array}$ & $\begin{array}{l}\text { Displays 3D equal power envelope around access points. } \\
\text { (Similar to Contour Coverage.) Provides visual of } \\
\text { propagation in all directions from a transmitter. In order to } \\
\text { generate the 3D contours it usually crosses multiple floors. }\end{array}$ \\
\hline Grid Coverage Mode & $\begin{array}{l}\text { Displays measurements as a color grid for a selected region. } \\
\text { Provides visual of power propagation through a region. } \\
\text { Capable of displaying power, SIR, SNR, relative power, and } \\
\text { throughput measurements. }\end{array}$ \\
\hline $\begin{array}{c}\text { Antenna Grid } \\
\text { Positioning Mode }\end{array}$ & $\begin{array}{l}\text { Assists in determining ideal locations for transmitting } \\
\text { antennas. This mode is much like the Antenna Positioning } \\
\text { mode however the user monitors the coverage of the entire } \\
\text { region rather than specific points. }\end{array}$ \\
\hline $\begin{array}{c}\text { Equidistant Boundary } \\
\text { Mode }\end{array}$ & $\begin{array}{l}\text { Displays predicted results at an equal distance from the } \\
\text { transmitting antenna. This distance can be changed in real- } \\
\text { time and the predicted values are updated. }\end{array}$ \\
\hline $\begin{array}{l}\text { Link Budget Table } \\
\text { Mode }\end{array}$ & $\begin{array}{l}\text { Provides power per channel, cable loss, antenna gain, mobile } \\
\text { transmit power and antenna gain, channel noise factor, } \\
\text { receiver noise factor, signal bandwidth, receiver sensitivity, } \\
\text { T-R separation distance, obstruction losses, and total } \\
\text { interference power at receiver measurements. These } \\
\text { calculations are made at specific points specified by the user. }\end{array}$ \\
\hline
\end{tabular}




\section{Overview of a Prediction within EnterprisePlanner ${ }^{\circledR}$}

In order to begin a prediction the drawing must be properly formatted for EnterprisePlanner $^{\circledR}$. All drawings, whether they were created in EnterprisePlanner ${ }^{\circledR}$ or not, must be formatted; this involves assigning material properties, aligning floors, and setting ceiling heights. Consider the simple floor plan in Figure 9. To format this drawing the Building Database Manipulator (BDM) Menu is used. This menu contains many tools for processing a drawing to be used in EnterprisePlanner ${ }^{\circledR}$. Figure 10 shows how a material type is selected then assigned to an object in the drawing.

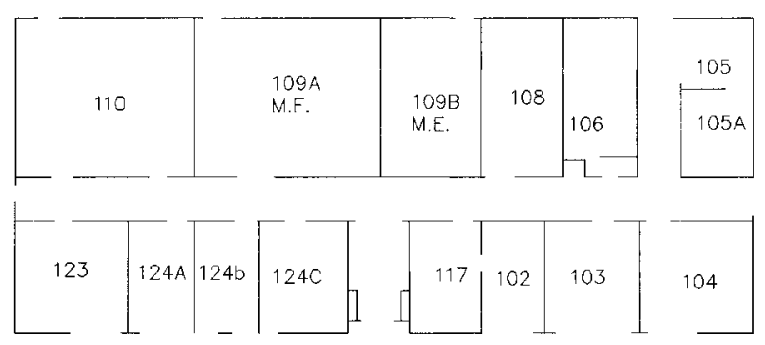

Figure 9: Simple floor plan.

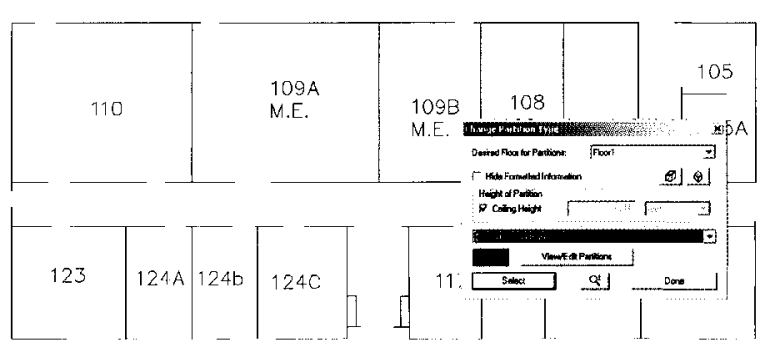

Figure 10: Formatting the floor plan.

Once all of the objects have been processed, the access point(s) can be placed in the drawing to simulate the wireless network. APs are placed using the Predictor Menu. In order to model the AP, EnterprisePlanner ${ }^{\circledR}$ needs specific information such as frequency range, channel set, transmit power, antenna type, antenna gain and its location. Figure 11 shows how these parameters are entered in to the drawing. 


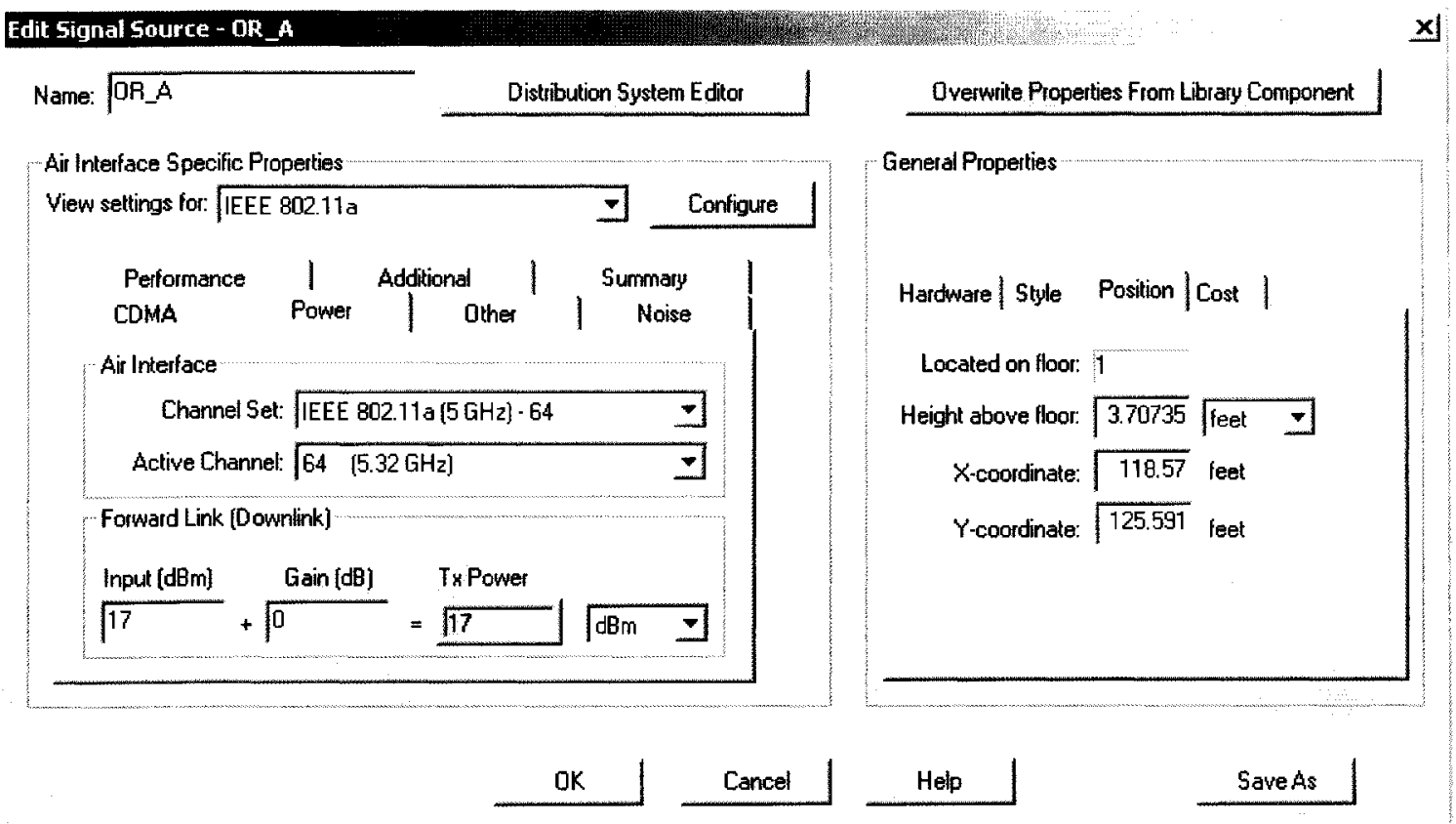

Figure 11: Entering AP parameters.

Predictions can be made after the AP is placed in the drawing. The prediction is initiated by selecting "Coverage Prediction..." from the Predictor Menu. Next the Prediction Mode is selected, in Figure 12 the Instant Point Mode is selected.

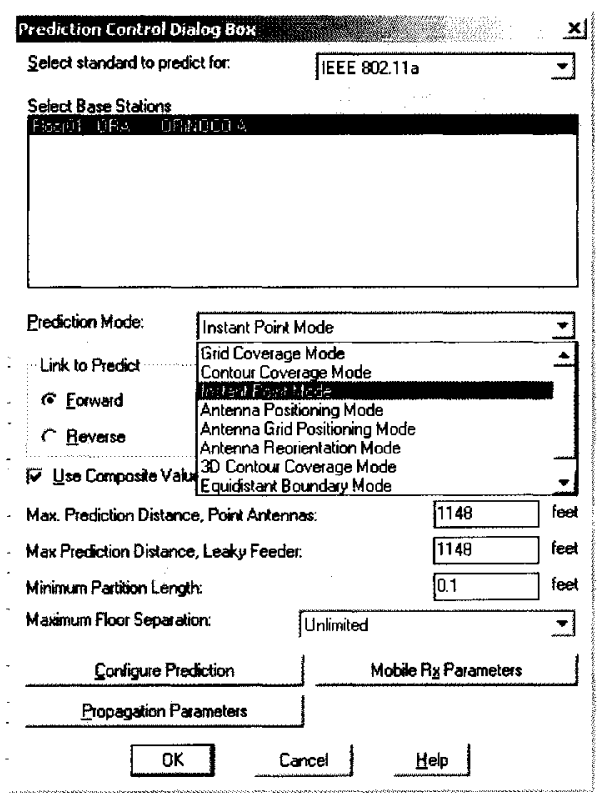

Figure 12: Selecting the prediction mode. 
Now the mobile receivers are set to match the receiver properties; this is shown in

Figure 13. Next the propagation parameters are then set, this includes selecting the path loss model as well as the type of path loss exponent. Figure 14 illustrates how these are selected. Lastly, if parameters other than the received power need to be predicted, they are selected through the 'Configure Prediction'. Once all of these parameters are set, the prediction can begin.

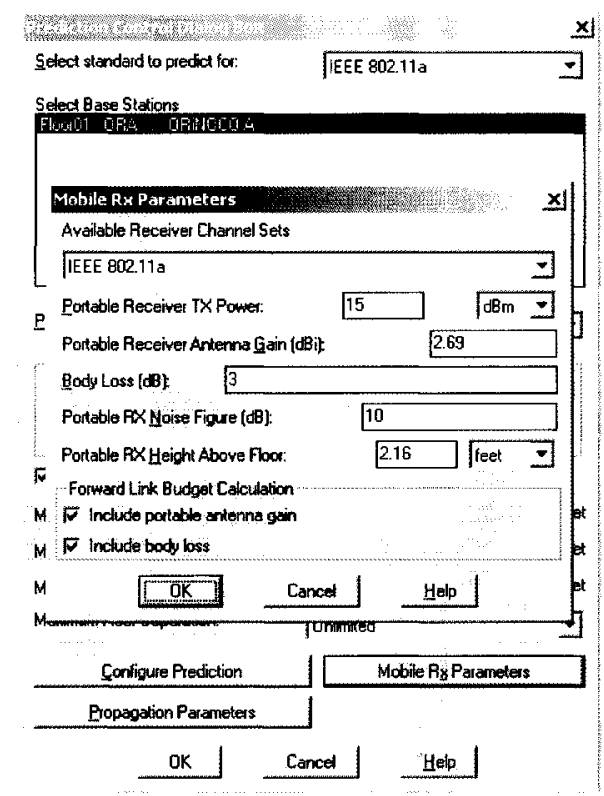

Figure 13: Setting the receiver parameters.
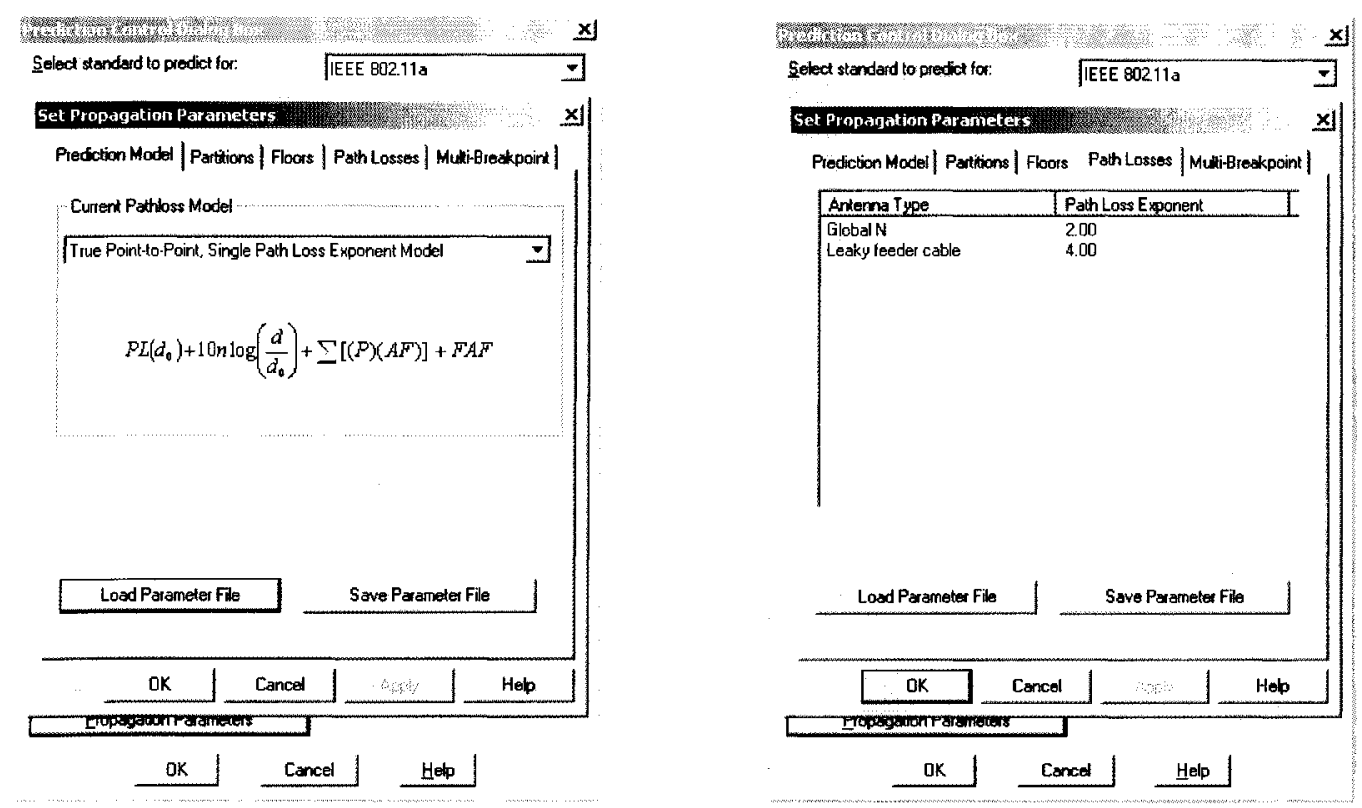

Figure 14: Selecting the path loss model and setting the path loss exponent. 
In the Instant Point Mode, the cursor acts as a mobile receiver. The location of the cursor determines the receiver location. Therefore a direct path is drawn from the transmitter to this point. The cursor can be moved to any location in the test area to display and record the Received Signal Strength Intensity (RSSI) value. Values are recorded by clicking in the environment. These values are stored as Watch Points, once all the watch points or data points have been placed the list of locations and measurements can be saved. Watchpoint files are stored as *.wch files which are easily imported into common spreadsheet software.

\section{Versions of EnterprisePlanner ${ }^{\circledR}$}

The work presented in this thesis was obtained through several versions of Wireless Valley, Inc. software applications. Several of the drawing files were first created in SitePlanner 7.0. Shortly after working in SitePlanner 7.0, SitePlanner 8.0 was released and research continued with this product. Later, EnterprisePlanner ${ }^{\mathbb{B}} 9.0$ was introduced from Wireless Valley, Inc. and was selected for future work. EnterprisePlanner ${ }^{\circledR}$ is an enhanced version of SitePlanner that allows for additional wireless systems to be designed and analyzed. All versions of SitePlanner and EnterprisePlanner ${ }^{\circledR}$ use the same prediction methods discussed herein and yield the same prediction results.

The latest version, EnterprisePlanner ${ }^{\circledR}$ 9.2, was recently released (a few weeks before the completion of this thesis). EnterprisePlanner ${ }^{\circledR} 9.2$ has an additional ray tracing prediction method. Due to time constraints this method was not used for predictions and its techniques were not rigorously investigated. Therefore, it is not presented in this chapter but its basic functionality is discussed in Chapter II. 


\section{CHAPTER IV}

\section{EM MEASUREMENTS OF 802.11A AND 802.11B WIRELESS NETWORKS INSIDE AIRCRAFT CABINS}

\section{Introduction}

The model created was to replicate an experimental study conducted in the spring of 2003, which examined 802.11 wireless communication network performance onboard several aircraft. The parameters of this experimental study were thoroughly examined in order to accurately compare the predicted results with the field results.

This chapter will discuss details of the experimental study whose data will be used to validate the results of the simulation. This includes the experimental set-up and test environment. Issues and concerns with the field tests are also addressed in this chapter. These measurements were recorded before this project was proposed. Therefore some parameters which were necessary for the simulation were not explicitly recorded at the time of testing. This chapter will initiate the discussion of these parameters; however, Chapter V will provide more detailed information concerning these parameters.

The field trial study [8] and other similar studies produced additional information beyond what is included in this chapter. Those results are not applicable to the objectives of this thesis or validation of the simulation and are not included herein.

\section{Experimental Set-up}

Wireless networks were configured and tested on four aircraft: three, two aisle Boeing planes, the 747-400, 767-300, 777-200 and one, single aisle Airbus 320. A WLAN was assembled for each aircraft using laptop computers and an Access Point (AP). The AP was connected to a laptop which acted as the server. The remaining laptops were used for collecting received power and throughput measurements throughout the cabin ([8] does not explicitly state how many laptops were used or if measurements were taken with several different laptops). Figure 15 and Figure 16 illustrate how the AP and laptop computers were positioned during data collection. 


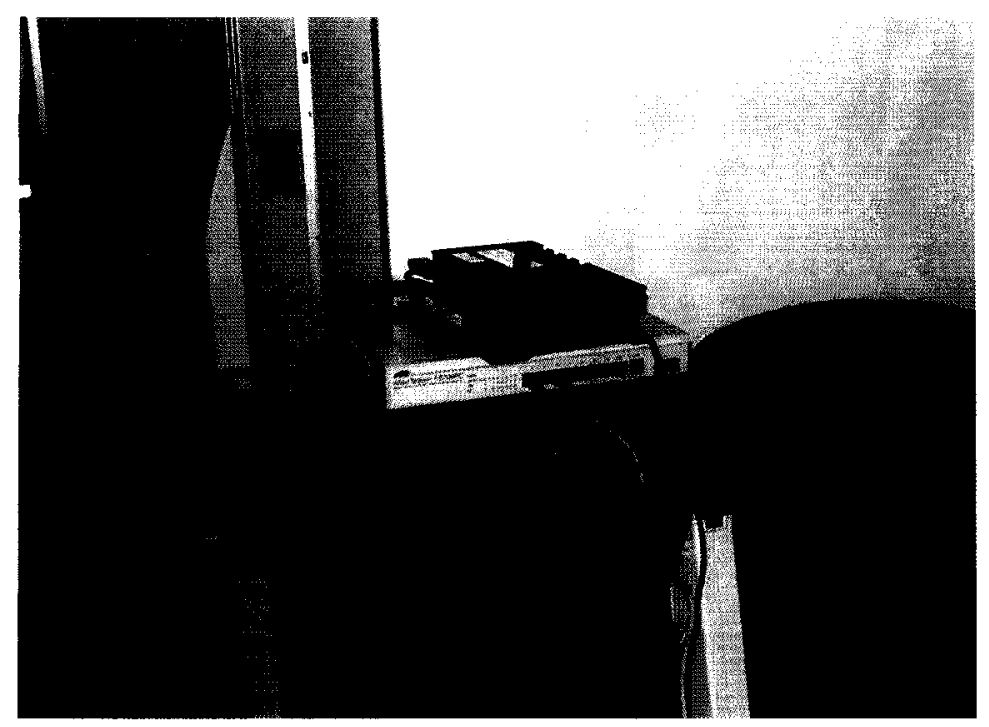

Figure 15: AP placement for testing in the A320

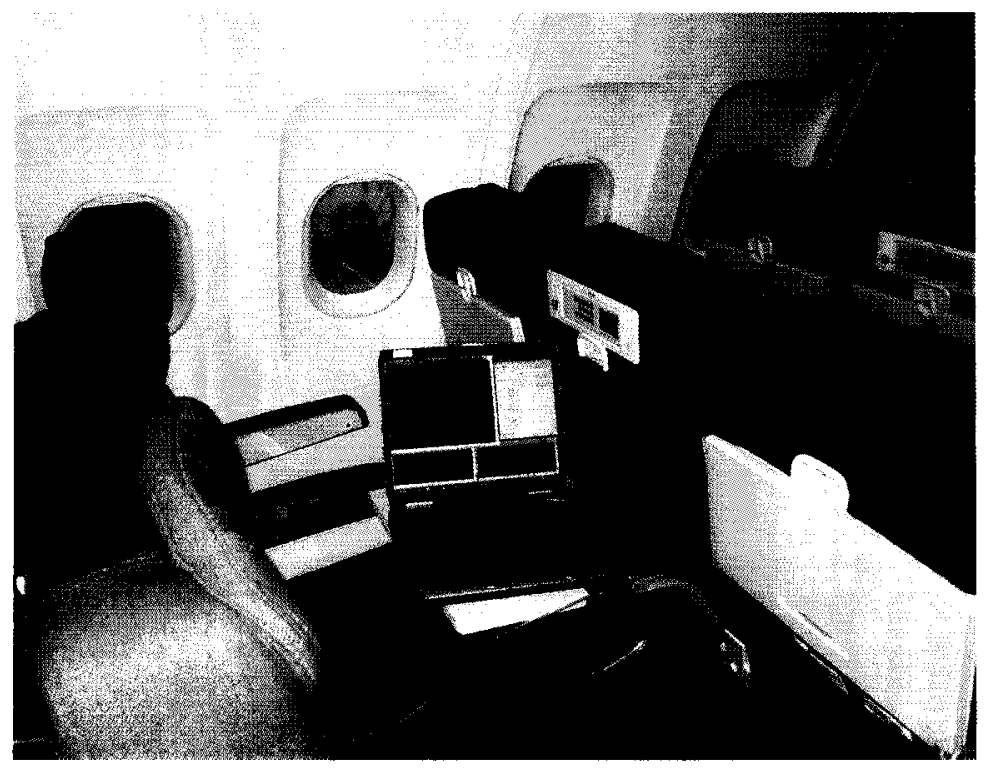

Figure 16: Mobile laptop computer running AirMagnet software for data collection.

A measurement was recorded at each seat row for a single aisle; however, the precise seat location and/or aisle (left or right, center aisle measurements were not recorded) was not noted in [8]. The receiver locations were only noted by the station number which is a distance from an arbitrary reference point located in the nose of the plane. The station number corresponds to the seat row where the measurement was taken but does not imply a seat location or aisle. 
Generally, the AP was situated in the front of the aircraft in first class seating. In the case of the A320, the AP was in the rear of the plane. Since this study was intended to gain an understanding of radio wave propagation within the fuselage rather than configure the plane for the most suitable WLAN, the AP was not placed in an ideal location.

Once the WLAN was configured, several aspects of the network could be evaluated. Several network and RF power measurements were made with the AirMagnet Wireless Network Analyzer. The AirMagnet tool uses the laptop's client card to calculate data rate, dropped frames, SNR, and received power. It was noted in [8] that the AirMagnet tool was uncalibrated and of unknown accuracy. However, it was considered adequate for the relative measurements and rough power measurements made during these field tests. Again, at the time of these tests researchers were not aware of the future applications of their data. Additional received power measurements were made with a spectrum analyzer. These measurements were considered to be a more repeatable and calibrated method compared to the AirMagnet measurements although the antennas used with the spectrum analyzer were not calibrated. Theoretically the two sets of received power measurements should have been similar and were expected to only differ in amplitude due to the uncalibrated nature of each test. The outcome was quite different. 


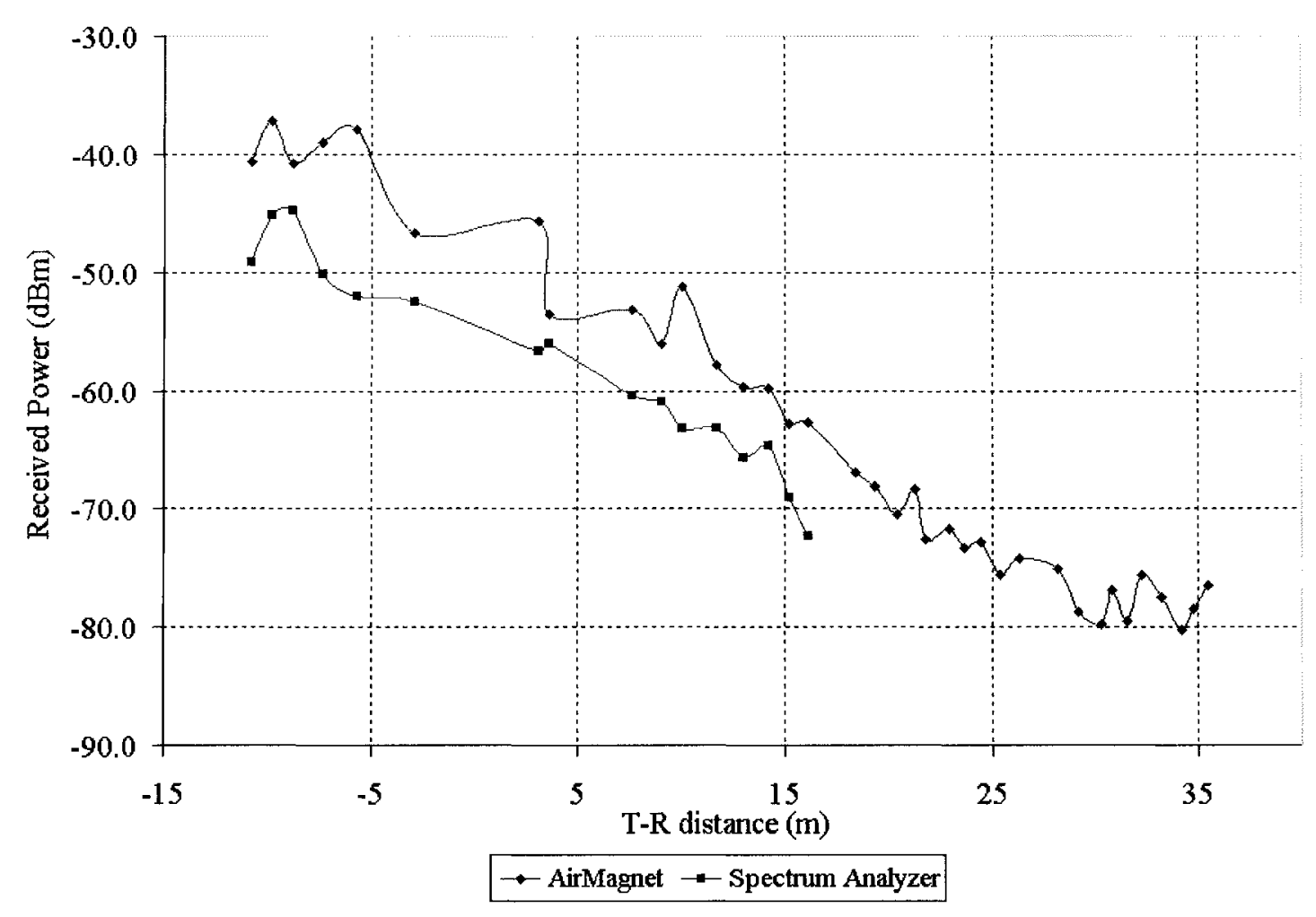

Figure 17: Comparison of 802.11a and $802.11 b$ results.

It is apparent from Figure 17 that the two devices gave contrasting results. This can be attributed to the differences in the measurement techniques of the two devices. The AirMagnet software uses spread spectrum technology whereas the spectrum analyzer measures power at each discrete frequency. Additionally, it was not specified whether these measurements were made simultaneously or not. It is possible that the spectrum analyzer and receiving laptop were not in the exact same location and/or that personnel acted as blocking agents to either instrument. As shown in Figure 17 there are instances where the client card illustrates a local maximum whereas the Spectrum Analyzer displays a minimum. This occurred at approximately $d=-8.75$ and $d=10$. 


\section{Test Environment}

The conditions inside the cabins during testing were neither ideal nor uniform. Each plane had a different configuration which was recorded in [8]. One uncontrollable condition was due to aircraft maintenance. Personnel were moving throughout the cabin performing routine maintenance during data collection. For two aircraft, the B767 and B777, some seats were removed and stacked on installed seats in another location. The resulting power measurements illustrated notable blockage. Since the maintenance crews needed access to the cabin, the doors could not be shut for all measurements. In some instances the doors were opened for ventilation. The door status was noted for each aircraft in [8]. Typically the aircraft were not powered up with the exception of a few systems in some cases. At no time did the researchers notice any significant differences in measurements due to power systems status.

\section{Concerns with the Test Environment}

Most EM prediction methods simulate an environment under ideal conditions. That is they usually do not include the effects of people or other EM sources (which may cause interference) in the environment. Additionally other objects in an indoor environment are likely to change position throughout daily activity, such as opening and closing doors. Therefore data collected in a more ideal setting will provide results more comparable to predictions. However, it is more desirable to collect data in a common setting so that measurements reflect the actual operational EM environment then compare these measurements to predictions. This allows for prediction results to be assessed on their ability to predict the actual EM environment under test.

As described in the previous section, data collection was conducted in a nonoperational environment. In flight, passengers are likely to impact the range of WLAN by providing additional (mobile) obstacles. Therefore these measurements only provide a general understanding of WLAN operation in the aircraft environment and neglect the passengers' RF effects such as absorption. 


\section{Challenges Associated with the Field Measurements}

Challenges for modeling this field study were encountered due to the fact that some parameters were not recorded at the time of testing (again, the researchers were not aware of the future applications of their work). This included the AP characteristics such as transmit power, antenna type and antenna gain. Additionally the transmitter (AP) and receiver locations were denoted by their seat location but specific heights or distances from a landmark were not recorded. Furthermore seat locations may differ from airline to airline, at the time of testing the station numbers were used to map seat locations and were taken from Boeing or Airbus specifications, not the airlines. All of these parameters had to be approximated based on the descriptions in [8] and will be discussed in Chapter V. 


\section{CHAPTER V}

\section{MODELING FOR PROPAGATION PREDICTION}

\section{Introduction}

The accuracy of the propagation prediction is dependent on the model of the test environment. Therefore, each aircraft and the access point were carefully researched to ensure that the model generation would portray the test environment as realistically as possible. EnterprisePlanner $\left.{ }^{(}\right)$incorporates the drawing capabilities of AutoCAD and drawing (*.dwg) files created in AutoCAD are easily imported into the program. Boeing and Airbus drawing files were not available due to security and copyright issues. Therefore, all four aircraft models were created in their entirety within EnterprisePlanner ${ }^{\circledR}$ from the basic dimensions and sketches openly available from the manufacturers websites [26],[28].

It was even more troublesome to locate parameters of the ORiNOCO AP2000 access point (now owned by Proxim). The Proxim website only supplied general information and their technical support services were unable to answer specific questions about the hardware. Eventually, the engineering department was able to supply the antenna pattern and confirm other details of the unit. Additionally, the FCC website was accessed to verify the findings as well as to provide information for the wireless card's receiving antenna.

It should be noted that for a previous publication, [27], the 802.11a propagation was thought to have two omni-directional antennas, based on misleading literature available on the Proxim website. Later it was discovered that the two antennas were actually dipoles, that when functioning together act as a single omni-directional antenna.

\section{Aircraft Under Test}

The Boeing and Airbus websites provided sketches and some basic dimensions of the aircraft which were used to calculate the necessary dimensions for the aircraft models. Some measurements were not explicitly provided on sketches or listed in the dimensions 
therefore approximations were made based on the provided scaled drawings. The table below summarizes the dimensions of each aircraft.

\begin{tabular}{|c|c|c|c|c|c|}
\hline \multicolumn{7}{|c|}{ Table 4: Summary of approximated aircraft dimensions. } \\
\hline Aircraft & $\begin{array}{c}\text { Height } \\
(\mathrm{m})\end{array}$ & $\begin{array}{c}\text { Width } \\
(\mathrm{m})\end{array}$ & $\begin{array}{c}\text { Length of } \\
\text { Cabin (m) }\end{array}$ \\
\hline
\end{tabular}

Two fuselage representations were developed for each plane: cylindrical and rectangular. This excludes the two-level B747 aircraft. For this fuselage only a rectangular fuselage was developed. The cylindrical model adhered to the dimensions described in Table 4. Due to limitations within EnterprisePlanner ${ }^{\circledR}$ a true cylinder could not be used for propagation predictions (this will be discussed in the Modeling Internal Components section). Consequently, an alternative model was created to retain the shape of the cylinder. The alternative cylindrical model consists of many rectangles stacked on top of one another to give the general shape of a cylinder, illustrated in Figure 18. The rectangular representations were initially included for comparison purposes and as an alternative to the more complex cylindrical model. However, after close inspection of the prediction methods it is clear that unless the receiver is outside of the aircraft, the exterior partitions are not included in the prediction. Furthermore, both models will be useful for future work utilizing the new ray tracing methods incorporated in the latest version of EnterprisePlanner $^{\circledR}$. The results of [16] illustrated that for larger aircraft, the EM 
propagation was comparable to an office environment. This further supports the use of rectangular representation for predictions.

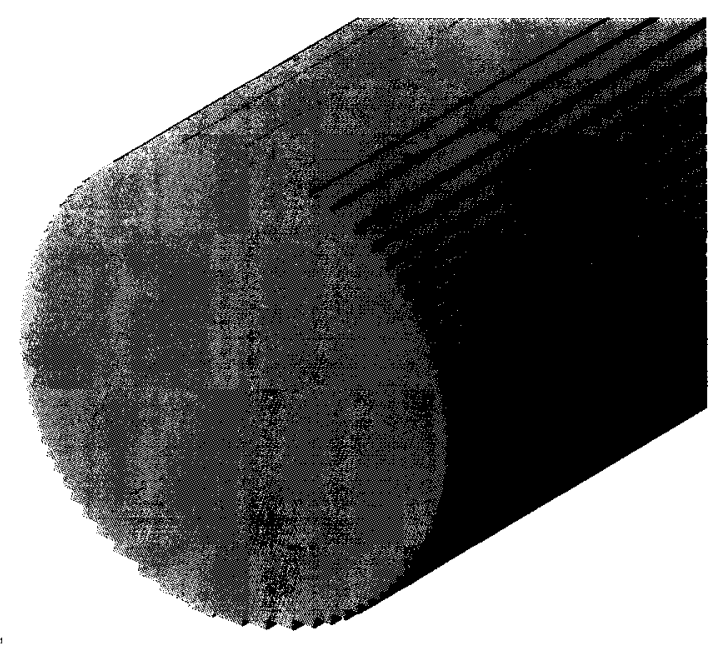

Figure 18: A close look at the cylindrical model.

Preliminary tests were conducted on empty fuselage models and indicated, as expected, that the results from the cylindrical and rectangular shaped models were very similar. This can be attributed to the fact that the direct path from the transmitter to the receiver did not pass through any partitions. Therefore, the predictions are based solely on the T-R distance and resemble free space path loss. The T-R distances in the rectangular and cylindrical models should be equal, and both models should yield the same results. Despite the similarities of the predictions with the two models, they were both used for future analysis. Although the results from the empty fuselage models followed the general trend of the experimental data, even more accurate results were expected if the interior of the plane was included in the model. This inclusion was expected to lead to more fluctuations in the power propagation as opposed to the smooth exponential like decay of the empty fuselage models.

\section{Modeling Interior Components}

The Layout of Passenger Accommodations (LOPA) taken from Boeing and Airbus files provided a representative floor plan for each aircraft. This was especially useful for drawing in EnterprisePlanner ${ }^{\circledR}$ which allows the user to trace a floor plan, scale it, and then extrude segments to their appropriate heights. The galleys and lavatories were 
traced from the LOPA and their height was set to the ceiling height. The LOPA includes the seat locations and the pitch (distance from seatback to seatback) but not any other specific seat information. This was found by contacting an aircraft seating manufacturer, $\mathrm{B} / \mathrm{E}$ Aerospace, Inc. which provided Boeing seat specifications that included dimensions of several classes of seats.

Drawing in EnterprisePlanner ${ }^{\circledR}$ creates planar surfaces which allows many objects to be simplified yet retain their EM properties. As mentioned above, drawing in EnterprisePlanner ${ }^{\circledR}$ consists of $2 \mathrm{D}$ in drawing then extruding each line segment to a specified height. Since objects must first be drawn in 2D, the cylindrical fuselage could not be drawn then extruded. The attenuation factors of the $3 \mathrm{D}$ materials are assigned to the planar surfaces, thus preserving all of the $3 \mathrm{D}$ characteristics of the material. Table 5 outlines the approximated attenuation factors for the aircraft components modeled in EnterprisePlanner ${ }^{\mathbb{B}}$. These values were approximated from EnterprisePlanner ${ }^{\mathbb{B}}$ s $s$ database of attenuation factors. The interior walls were expected to be slightly less than drywall and the seat components were estimated to be slightly less than cubicle walls.

Table 5: Assigned attenuation factors.

\begin{tabular}{|l|c|}
\hline \multicolumn{1}{|c|}{ Partition Type } & Attenuation Factor \\
\hline Exterior Walls (Fuselage) & $120 \mathrm{~dB}$ \\
\hline Interior Walls (Galleys, lavatories) & $2 \mathrm{~dB}$ \\
\hline Seat Components (Cushions, armrests) & $1 \mathrm{~dB}$ \\
\hline
\end{tabular}



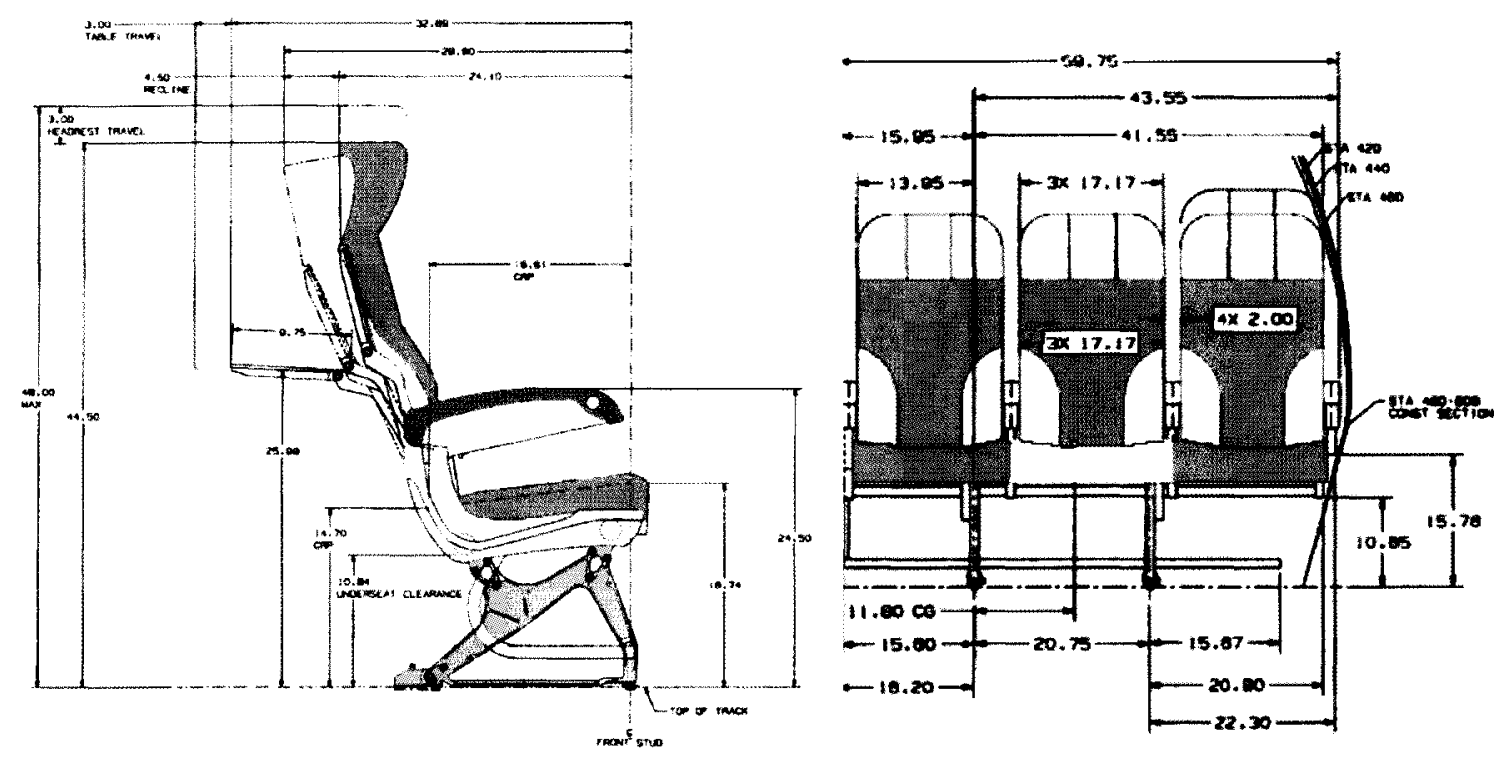

Figure 19: Dimensions of an economy class seat provided from B/E Aerospace, Inc.

Table 6: Approximated seat dimensions.

\begin{tabular}{|c|c|c|c|}
\hline Seat Component & Economy Class & Business Class & First Class \\
\hline Seatback height & $3.7 \mathrm{ft}$ & $3.5 \mathrm{ft}$ & $3.5 \mathrm{ft}$ \\
\hline Chair Width & 1.4 & 1.6 & 1.9 \\
\hline Seat height & 1.4 & 1.4 & 1.2 \\
\hline Arm rest height & .9 & 2 & 1.5 \\
\hline Arm rest width & .2 & .4 & .45 \\
\hline
\end{tabular}

Tutorial: How to Create a Seat in EnterprisePlanner ${ }^{\circledR}$

Creating a seat in EnterprisePlanner ${ }^{\circledR}$ consists of simplifying the seat into basic rectangles with different heights. The attenuation factors are assigned using the BDM menu as discussed in Chapter III. When completed the simplified seat will loosely resemble an actual seat but will contain all the necessary data for a power propagation prediction. First the overhead or top view of the seat is drawn, as shown in Figure 20. 


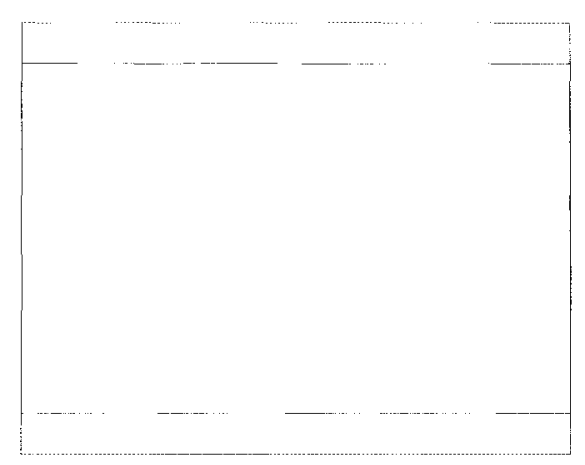

Figure 20: 2D, top view of seat.

Next the BDM module is used to assign material properties and extrude the seat components. Each line segment in Figure 21 is specified a material name and associated attenuation factor as well as the ceiling height. Figure 22 illustrates how each segment is extruded. The final outcome for the seat simplification is shown in Figure 23.
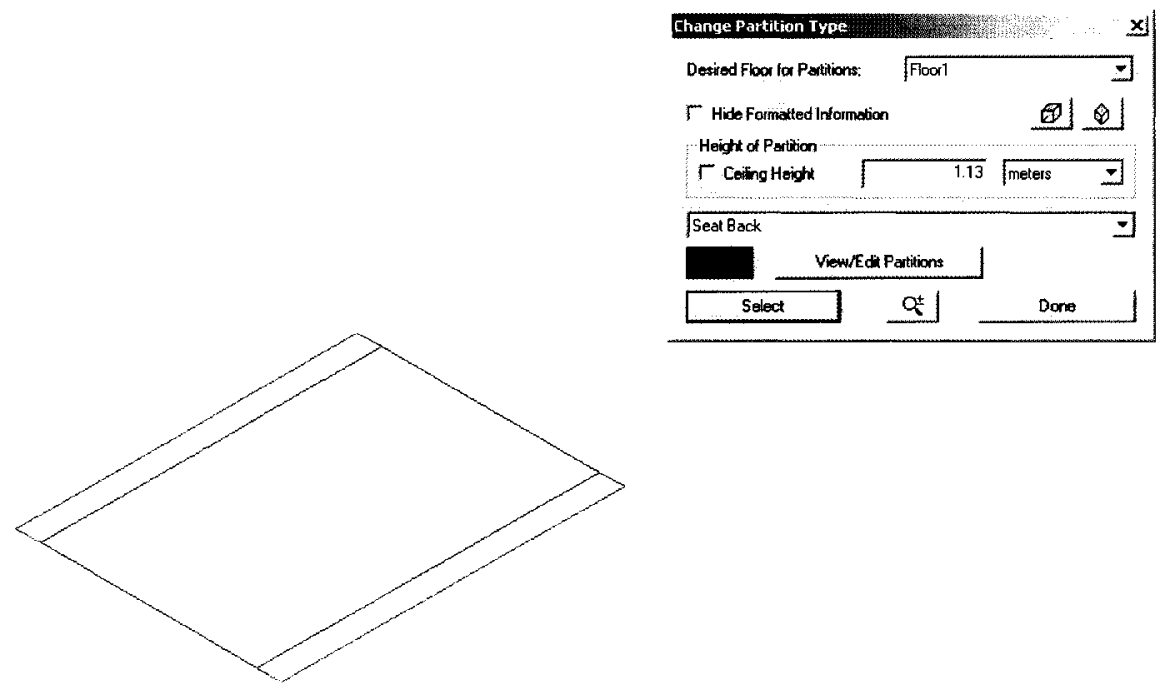

Figure 21: 3D view seat before formatting. 


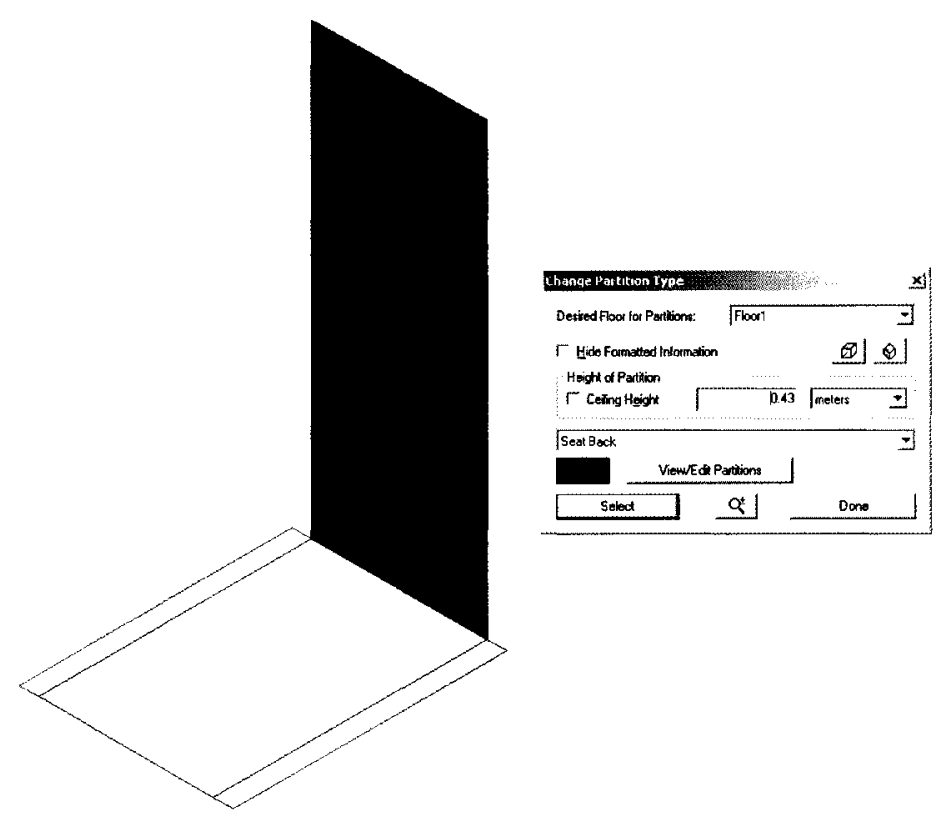

Figure 22: 3D view seat with one segment formatted.

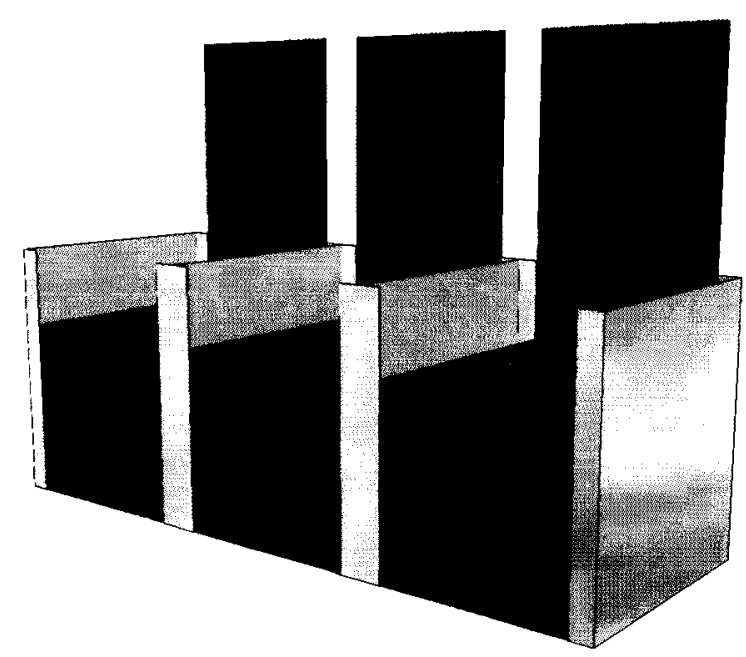

Figure 23: 3D view of simplified seat.

\section{Aircraft Models}

As discussed in the previous section the drawing capabilities of EnterprisePlanner ${ }^{\circledR}$ were used to create the fuselage models. The $3 \mathrm{D}$ images of each aircraft appear similar and will seem repetitive presented along side each other; therefore, 
the B767 cylindrical and rectangular representations as well as the B747 representation will be presented in $3 \mathrm{D}$. The remaining aircraft models will be compared in $2 \mathrm{D}$ against the LOPA provided from Boeing and Airbus websites. In Figure 26 through Figure 29 the red marker represents the transmitter or AP location.

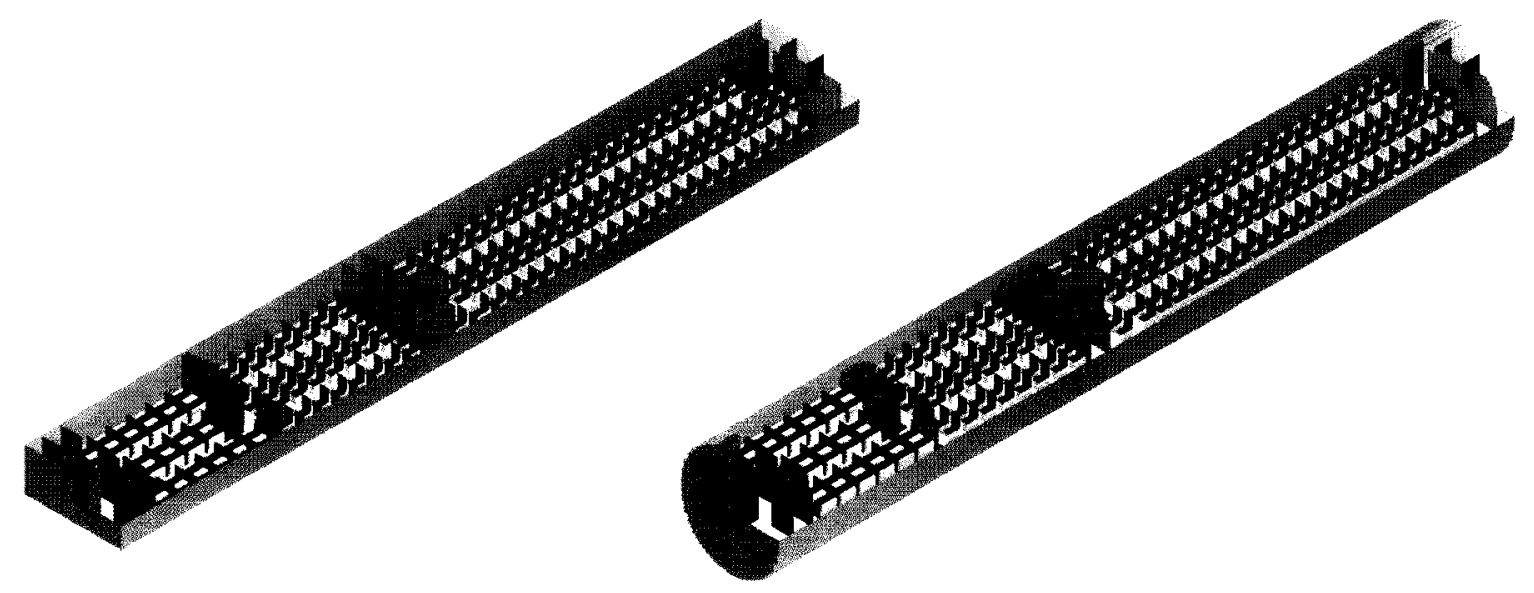

Figure 24: B767 Rectangular and Cylindrical representations featuring interior components (3D SW view with a quarter section removed for illustration purposes)

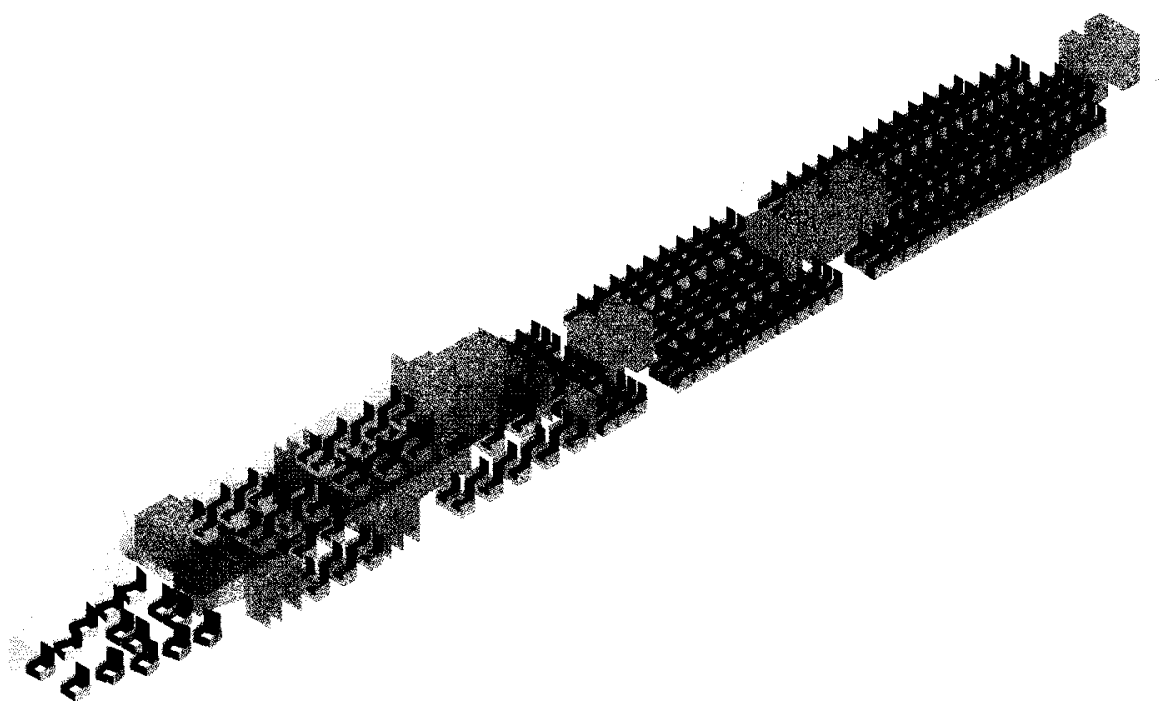

Figure 25: 3D view of $B 747$ representation featuring interior components. 

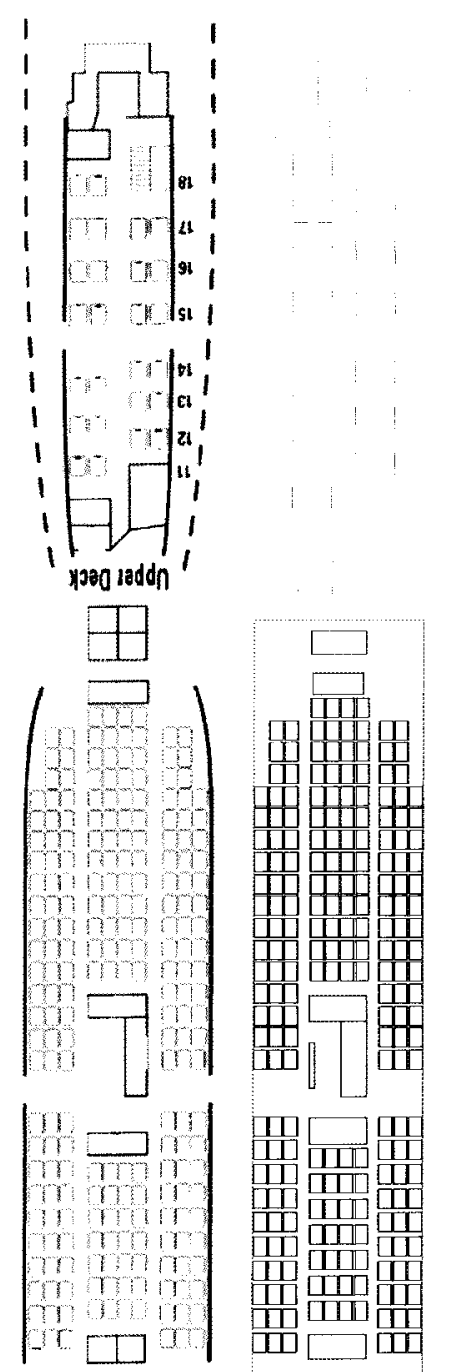

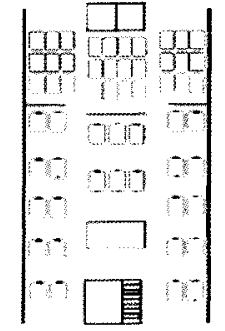

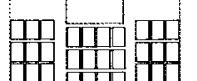

田㭰曲

एप

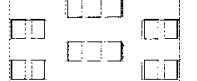

ڤ్

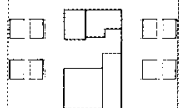

$\square$
$\square \square$
$\square \square$
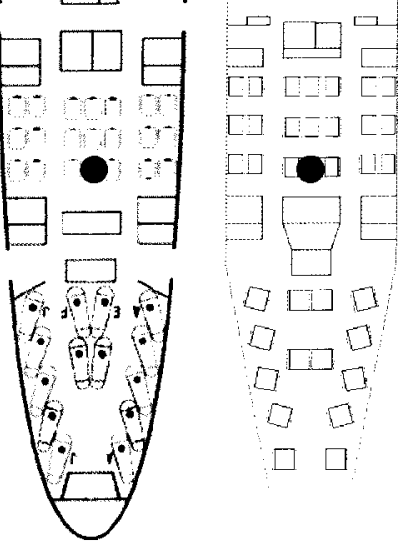

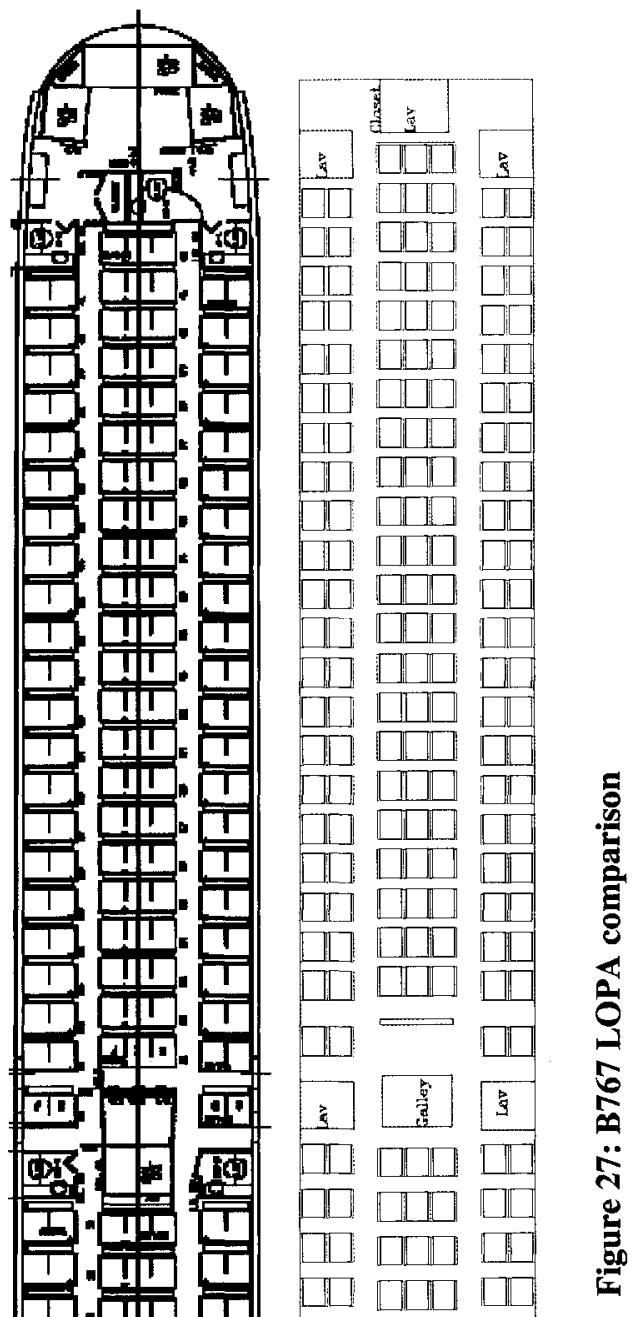

$\square \square \square \square \square \square]$

$\square \square \square \square \square \square$

$\square \square \square \square \square \square$

$\square \square \square \square \square \square$

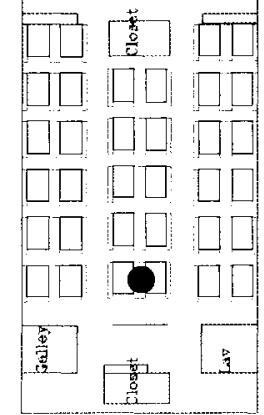




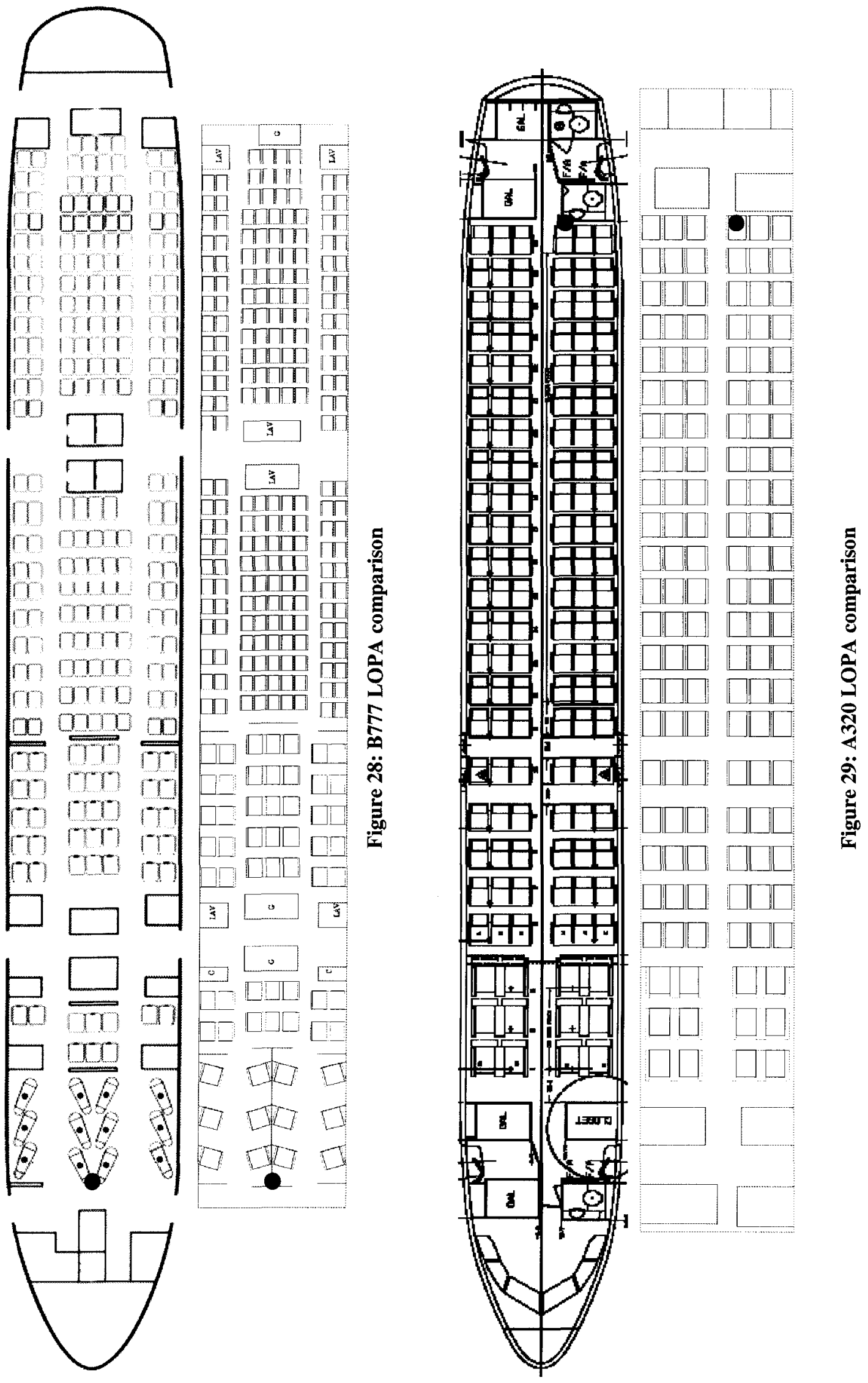




\section{Access Points}

EnterprisePlanner ${ }^{\circledR} 9.0$ incorporated a "Place WLAN Access Point" feature which simplified the process for modeling APs. Previously, the APs were created by attaching antennas to base stations. This process had to be repeated for each drawing file (for each plane model). The new feature allows an AP to be designed and saved for later use. This feature also allowed for antennas to be specified as integrated or detachable, which is useful for modeling the integrated antenna for $802.11 \mathrm{~b}$ propagation. Chapter III discussed how to place an AP in EnterprisePlanner ${ }^{\circledR}$.

The ORiNOCO AP2000 can operate at 802.11 a or $802.11 \mathrm{~b}$ standards. In order to properly model the AP2000, two different APs were designed in EnterprisePlanner ${ }^{\circledR}$ for each wireless standard. To create the AP there are several parameters that need to be specified. The table below outlines these parameters.

Table 7: Summary of ORiNOCO AP2000 parameters.

\begin{tabular}{|l|l|l|}
\hline & 802.11a Mode & 802.11b Mode \\
\hline Transmit Power & $17 \mathrm{dBm}$ & $15 \mathrm{dBm}$ \\
\hline Antenna Type & Omni-directional (detachable) & Dipole (integrated) \\
\hline Antenna Gain & $5 \mathrm{dBi}$ & $3 \mathrm{dBi}$ \\
\hline Antenna Polarization & Vertical & Vertical \\
\hline
\end{tabular}

The actual AP unit was not available for testing; therefore, all of the above information was found through contacting the manufacturer or distributor and locating FCC files.

Antenna patterns for both the integrated dipole and the omni-directional were found and incorporated into the design of the AP. The integrated dipole for $802.11 \mathrm{~b}$ mode is a fairly simple antenna. It was modeled by selecting a "GENERIC" antenna from EnterprisePlanner ${ }^{\circledR} \mathrm{s}$ database which has the same gain and polarization. The omnidirectional antenna was slightly more complex and required a new antenna to be created within EnterprisePlanner ${ }^{\circledR}$. Using the antenna pattern editor, Figure 30, the pattern provided by the manufacturer was recreated. The polarization and frequency were also specified using the antenna pattern editor. 


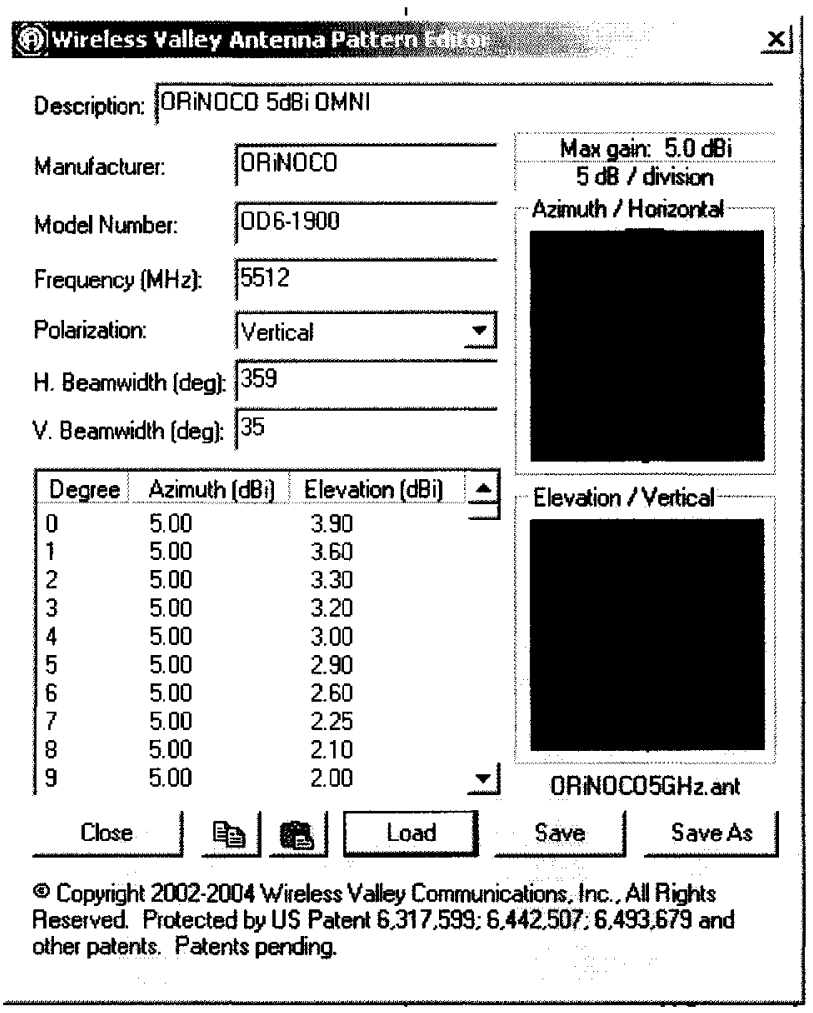

Figure 30: Creating the 5GHz Omni-directional antenna.

Once the two AP models were created they were inserted into the aircraft drawing files. Their positions are specified by an $(x, y)$ location and the height above the floor. These locations were estimated by the descriptions from [8] (no specific heights, distances, etc. were noted in their report). The general AP locations are shown in Figure 26 through Figure 29 as a red marker and their approximated heights are summarized in Table 8. 
Table 8: AP location approximations.

\begin{tabular}{|l|l|l|}
\hline Plane & Description & Approximation \\
\hline B747 & Mounted on back of seat 6D & $\begin{array}{l}\text { Placed at First class seat height (3.5 ft) at the } \\
\text { corner of seat 6D closet to the aircraft's } \\
\text { centerline }\end{array}$ \\
\hline B767 & Mounted on back of seat 1E & $\begin{array}{l}\text { Placed at the First class seat height (3.5 ft) at } \\
\text { the corner of seat 1E closest to the aircraft's } \\
\text { centerline }\end{array}$ \\
\hline B777 & $\begin{array}{l}\text { Mounted on bulkhead between } \\
\text { the footrests of seats 1E and 1F }\end{array}$ & $\begin{array}{l}\text { Placed at a height of 1.5 } \mathrm{ft} \text { at the aircraft's } \\
\text { centerline, 2 } \mathrm{ft} \text { from row 1 }\end{array}$ \\
\hline A320 & Mounted on back of seat 26C & $\begin{array}{l}\text { Placed at the Economy class seat height }(3.7 \\
\mathrm{ft}) \text { at the corner of seat 26C closet to the } \\
\text { aircraft's centerline }\end{array}$ \\
\hline
\end{tabular}

\section{Receivers}

Information for the receiver is entered at the start of each prediction. This was illustrated in Chapter III, Figure 13. The receiver antenna gain and body loss were both included in the predictions. Body loss is the absorption from the user (human factor), typically $3 \mathrm{~dB}$. Two types of power measurements were taken in [8]. Ideally simulations for both types of receivers should be completed; however, information about the spectrum analyzer antenna was not provided. All simulations were done with the information researched on the ORINOCO 802.11a/b ComboCard WLAN client card used in the mobile laptop. EnterprisePlanner ${ }^{\mathbb{B}}$ associates an isotropic antenna with the receiver; therefore, only the transmit power and gain are specified. The table below lists the client card's parameters. 
Table 9: Receiver Characteristics.

\begin{tabular}{|l|l|}
\hline Product Name & ORiNOCO a/b Gold Client Card \\
\hline Manufacturer & ORiNOCO (now owned by Proxim) \\
\hline Part Number & 8460 \\
\hline FCC ID & IMR WLPCE24H \\
\hline Transmit Power & $15 \mathrm{dBm}$ \\
\hline Antenna Gain & $2.69 \mathrm{dBi}$ \\
\hline Receiver Height & Seat tray height, approximately $2.16 \mathrm{ft}(.66 \mathrm{~m})$ \\
\hline Receiver Locations $(\mathbf{x}, \mathbf{y})$ & Approximated as the center point of each seat edge \\
\hline
\end{tabular}

\section{Summary}

In this chapter the methods for modeling the aircraft and APs were presented. The aircraft and APs were carefully researched and the models were created as accurately as possible. Any simplifications or assumptions have been discussed and explained. These details laid the necessary foundation for understanding the differences between each model. This will aid in the discussion of the results for each model in the following chapter. 


\section{CHAPTER VI}

\section{PREDICTION RESULTS AND ANALYSIS}

\section{Introduction}

This Chapter will begin by discussing how to experimentally find the path loss exponent, $n$. The path loss exponent is set for all predictions and is a vital parameter to the prediction. Once the path loss exponent issue is addressed, the prediction results and analysis for all the aircraft will be presented.

\section{Calculating the Path Loss Exponent, $n$}

As discussed in Chapters II and III, the path loss exponent is a quantity related to the rate of decay of the signal. By examining the relationship in Equation (14) it is clear that one method to compute the path loss exponent from measured data is to plot the power versus T-R distance on a log-log plot, then calculate the linear regression. This method is outlined in [15] The slope of the line is the path loss exponent. The results from [8] were used to calculate the path loss exponent. One complication is that the distance from the AP to the mobile receiver was not recorded at the time of testing. These distances were found using the EnterprisePlanner ${ }^{\circledR}$ models and estimating the receiver location (seat and position). Only the seat row was recorded in [8] therefore all distances are estimated from the middle seat of the outer rows (in the case of two seats in the outer row, the distances were measured from the middle armrests). It was assumed that the receiver would be located at the seat-back tray height above the middle edge of the seat. Figure 31 and Figure 32 show the measured data against the linear regression and Table 10 summarizes the calculated path loss exponents for each plane, wireless standard and measurement type. The measurements recorded with the spectrum analyzer are referred to as 'Spectrum Analyzer' and the measurements recorded with the laptop's client card and AirMagnet software are referred to as 'AirMagnet'. 


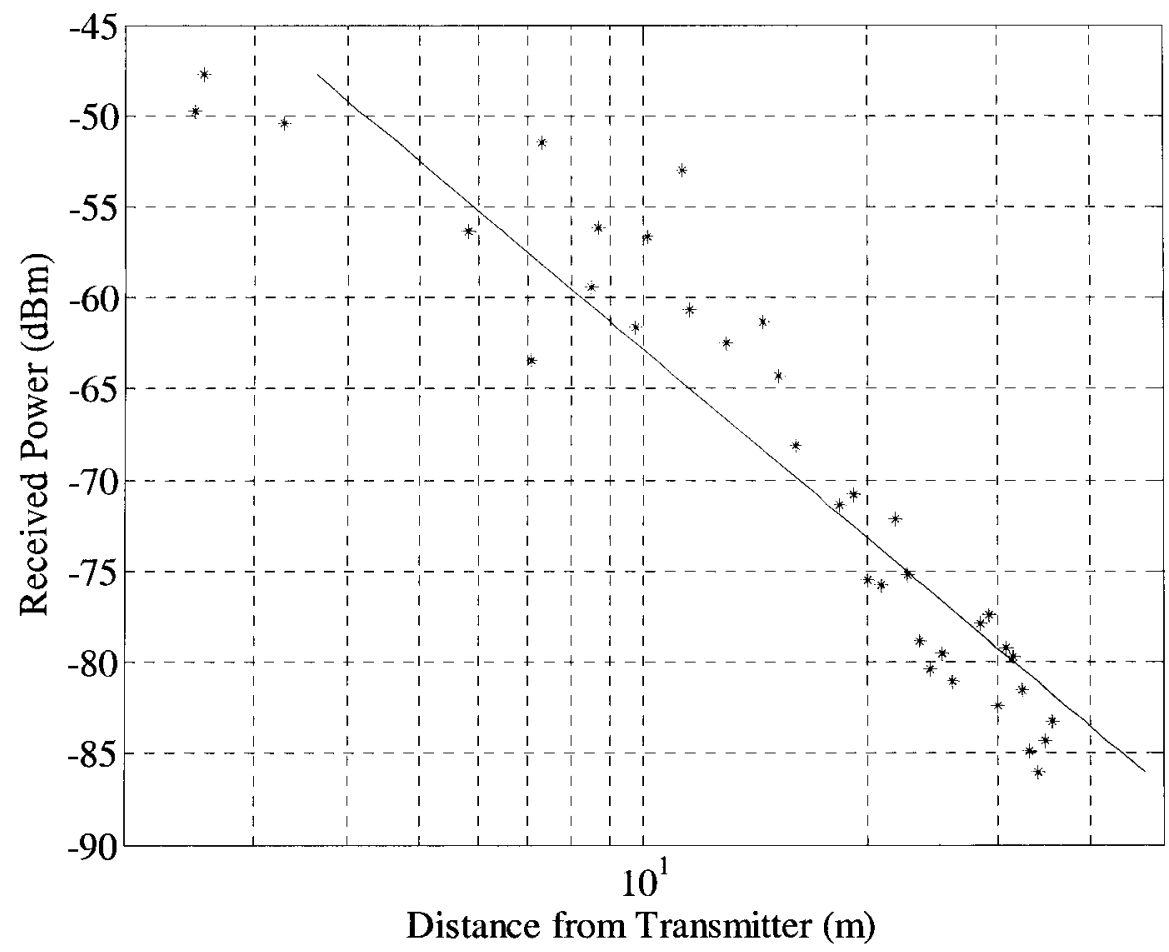

Figure 31: Calculation of path loss exponent at $5 \mathrm{GHz}$ from AirMagnet measurements for the B747.

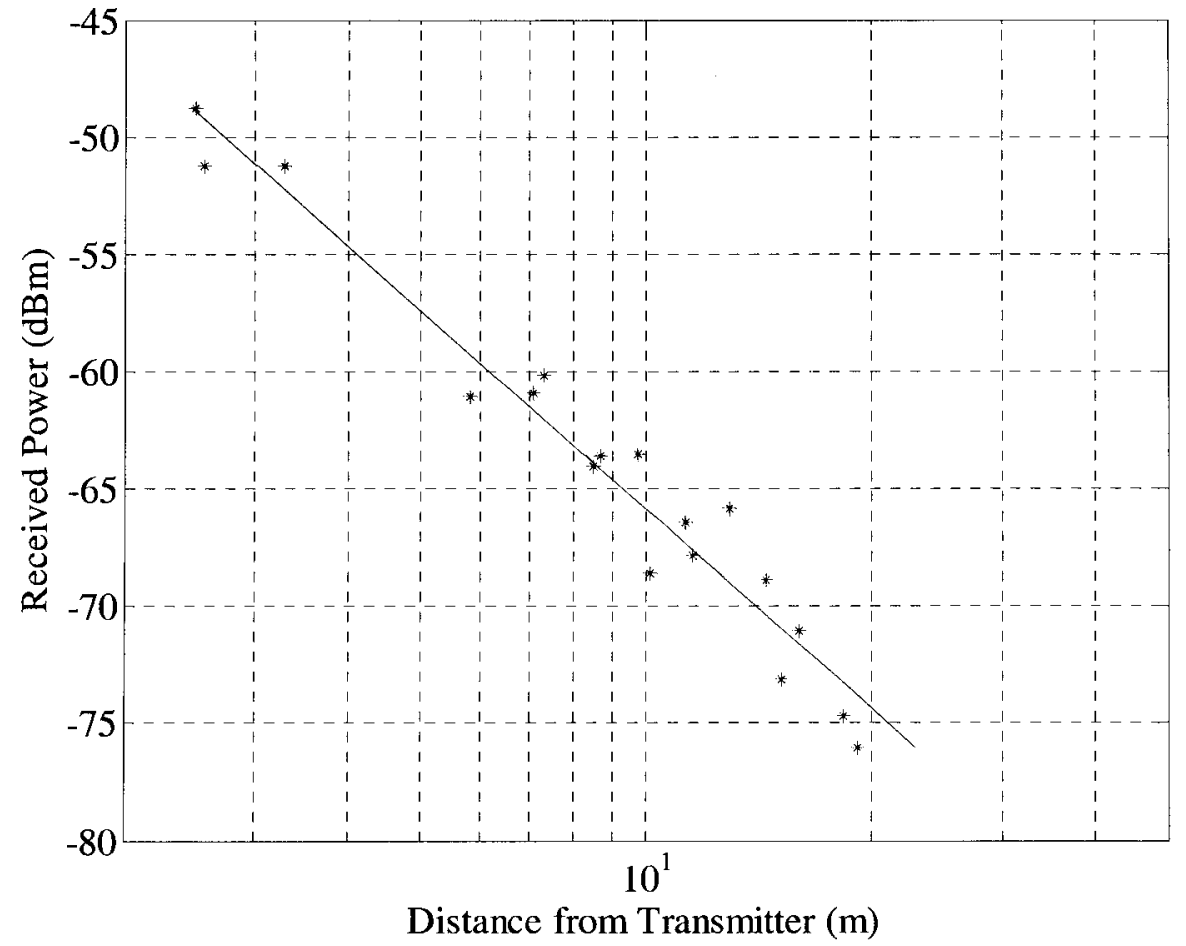

Figure 32: Calculation of path loss exponent at $5 \mathrm{GHz}$ from Spectrum Analyzer measurements for the $B 747$. 
Table 10: Summary of estimated path loss exponents.

\begin{tabular}{|l|c|c|c|c|}
\hline Aircraft & \multicolumn{2}{|c|}{ 802.11a (5 GHz) } & \multicolumn{2}{c|}{$\mathbf{8 0 2 . 1 1 b} \mathbf{( 2 . 4} \mathbf{~ G H z )}$} \\
\hline B747 & 3.44 & 2.81 & 2.78 & 3.4 \\
\hline B767 & 3.75 & 2.66 & 2.5 & 3.15 \\
\hline B777 & 2.84 & 2.78 & 2.84 & 3.10 \\
\hline A320 & 3.2 & N/A & 3.32 & N/A \\
\hline & AirMagnet & Spectrum Analyzer & AirMagnet & Spectrum Analyzer \\
\hline
\end{tabular}

The path loss exponents calculated are similar to those calculated for indoor environments. These values suggest that the environment inside the aircraft cabin is similar to that of an indoor building environment. This is somewhat unexpected considering the shape and materials of the aircraft. However, it seems that at high frequencies the exterior of the aircraft for large aircraft may not be as significant as suggested from radio propagation theory. For smaller aircraft or at lower frequencies, the effects of the exterior of the aircraft are likely to be more substantial.

\section{Prediction Results}

This section will be divided into subsections to allow results of each aircraft to be presented independently. The analysis of these results will be provided in the following section.

The results are presented as a graphical comparison of the with field measurements. A plot of predicted throughout the entire cabin is also presented. In addition, some statistical analysis is also presented. For each test the Mean Absolute Error (MAE) and Root Mean Square Error (RMSE) are calculated. The equations that describe these calculations are given in (20) and (21). These results provide further inference about the accuracy of the prediction.

$$
M A E=\frac{\sum\left|P_{M}-P_{P}\right|}{N} \quad[d B m]
$$

where $P_{M}$ is the measure power, $P_{P}$ is the predicted power and $N$ is the total number of measurements. 


$$
R M S E=\sqrt{\frac{\sum\left(P_{M}-P_{P}\right)^{2}}{N}} \quad[d B m]
$$

The $M A E$ calculation will provide the average error between the predicted and measured received power measurements. The RMSE calculation provides further analysis of the differences between the predicted and measured results. RMSE exaggerates the cases where the error between the measured and predicted measurements is significant. For both calculations, values closer to zero are more accurate. MAE and RMSE values of zero reflect a prediction identical to measured results. In a case where the MAE of two predictions are equal but the RMSE differ, the prediction with a lower RMSE value would be a more accurate prediction.

\section{Explanation of Graphs}

The received power and predicted received power are graphed against the distance from the transmitter to the receiver. The T-R separation distance corresponds to the seat location as discussed in the previous section. For the B747 the negative T-R distances correspond to a receiver location before the transmitter which is to the left of the transmitter in reference to Figure 26 through Figure 29.

There are several predicted results. The 'Rectangular' result refers to the rectangular representation including interior components and the 'Cylindrical' result follows similarly. The remaining two results are essentially free space propagation with the path loss exponent set to the calculated path loss exponents (the first $n$, in green, corresponds to $n_{\text {AirMagnet }}$ and the second, in purple, corresponds to $n_{\text {SpectrumAnalyzer }}$ ). However, they were found using the rectangular representation with the Distance Dependent Path Loss Model (no difference was noted if the exterior walls were removed).

Predicted results from Grid Coverage Mode are also presented. These results can not be verified with the information in [8] and are provided for illustration purposes only. All of the Grid Coverage Mode results share the same legend shown in Figure 33. 
Grid Coverage, Forward Link

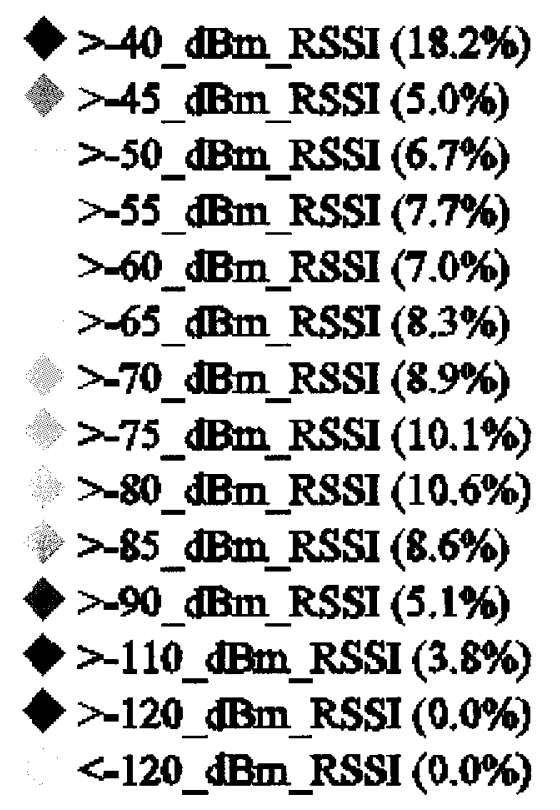

Figure 33: Legend for Grid Coverage results. 
B747 Results

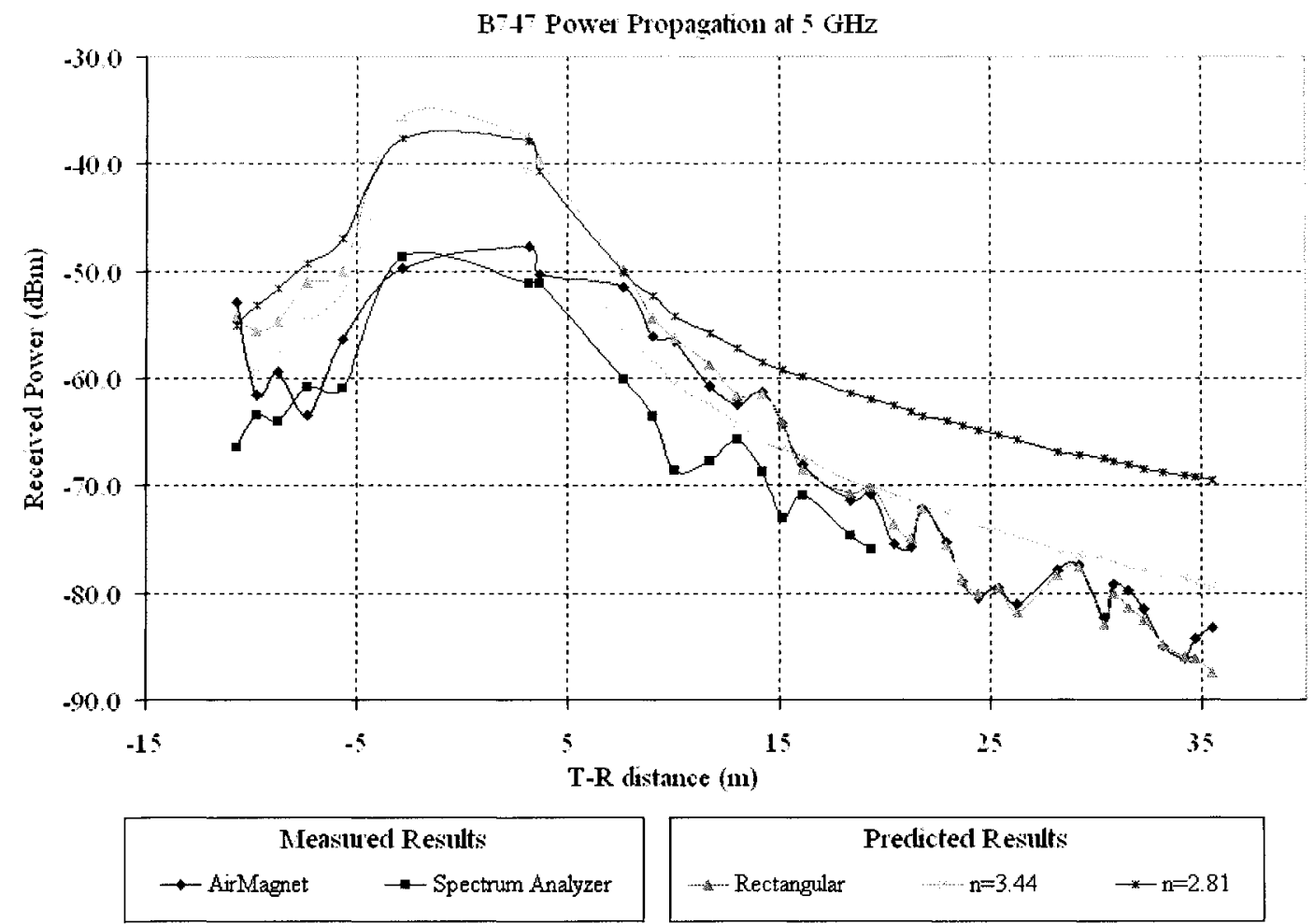

Figure 34: B747 results for 802.11a (excluding Floor 2 of the aircraft).

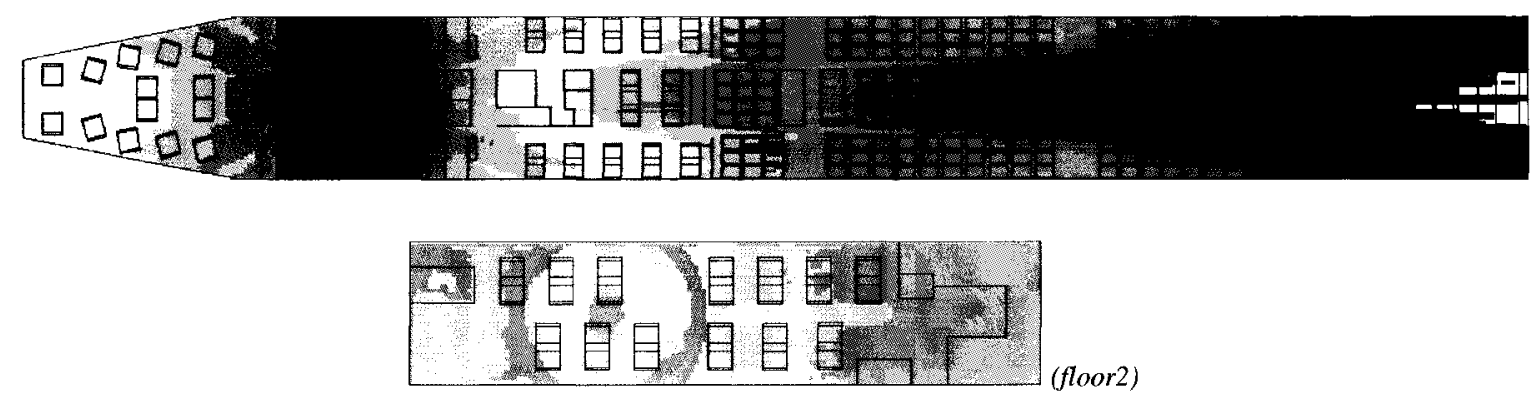

Figure 35: B747 802.11a power propagation using Grid Coverage Mode. 


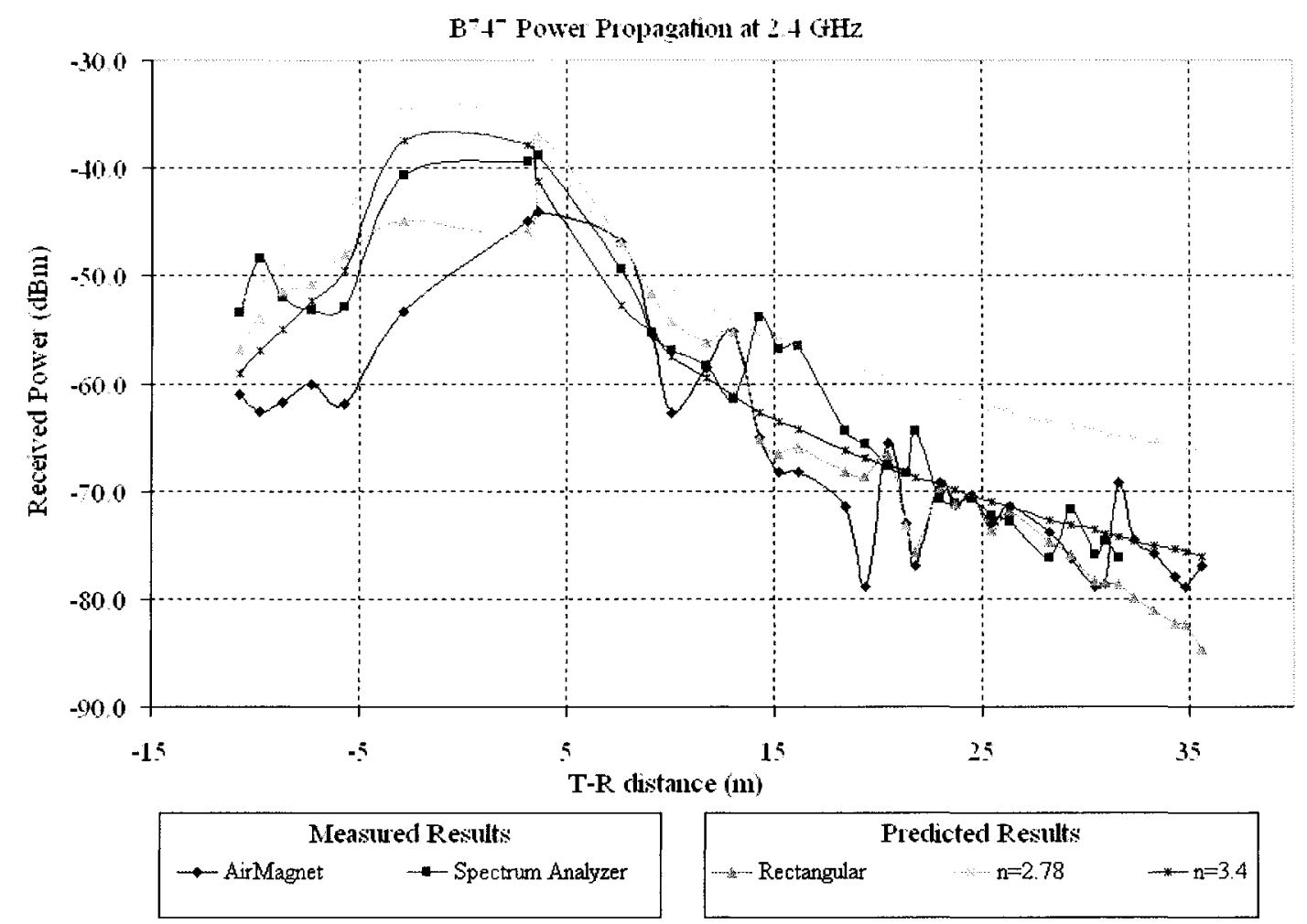

Figure 36: B747 results for 802.11b (excluding Floor 2 of the aircraft).
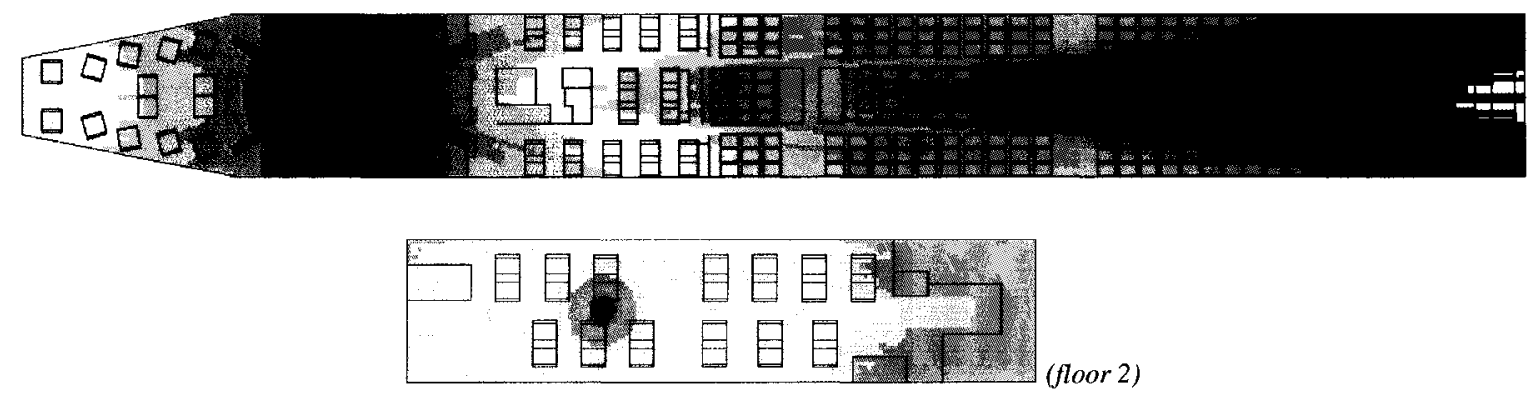

Figure 37: B747 802.11b power propagation using Grid Coverage Mode.

Table 11: Statistical analysis of $B 747$ results.

\begin{tabular}{|l|r|r|r|r|l|}
\hline & \multicolumn{2}{|c|}{ 802.11a } & \multicolumn{2}{c|}{ 802.11b } & \\
\hline Rectangular & 2.24 & 4.12 & 3.42 & 5.01 & AirMagnet \\
\hline Rectangular & 7.87 & 8.66 & 3.84 & 4.90 & Spectrum Analyzer \\
\hline$n_{\text {AirMagnet }}$ & 4.52 & 5.18 & 10.96 & 11.83 & AirMagnet \\
\hline$n_{\text {Spectrum Analyzer }}$ & 12.94 & 11.94 & 3.40 & 4.41 & Spectrum Analyzer \\
\hline & MAE & RMSE & MAE & RMSE & \\
\hline
\end{tabular}


B767 Results

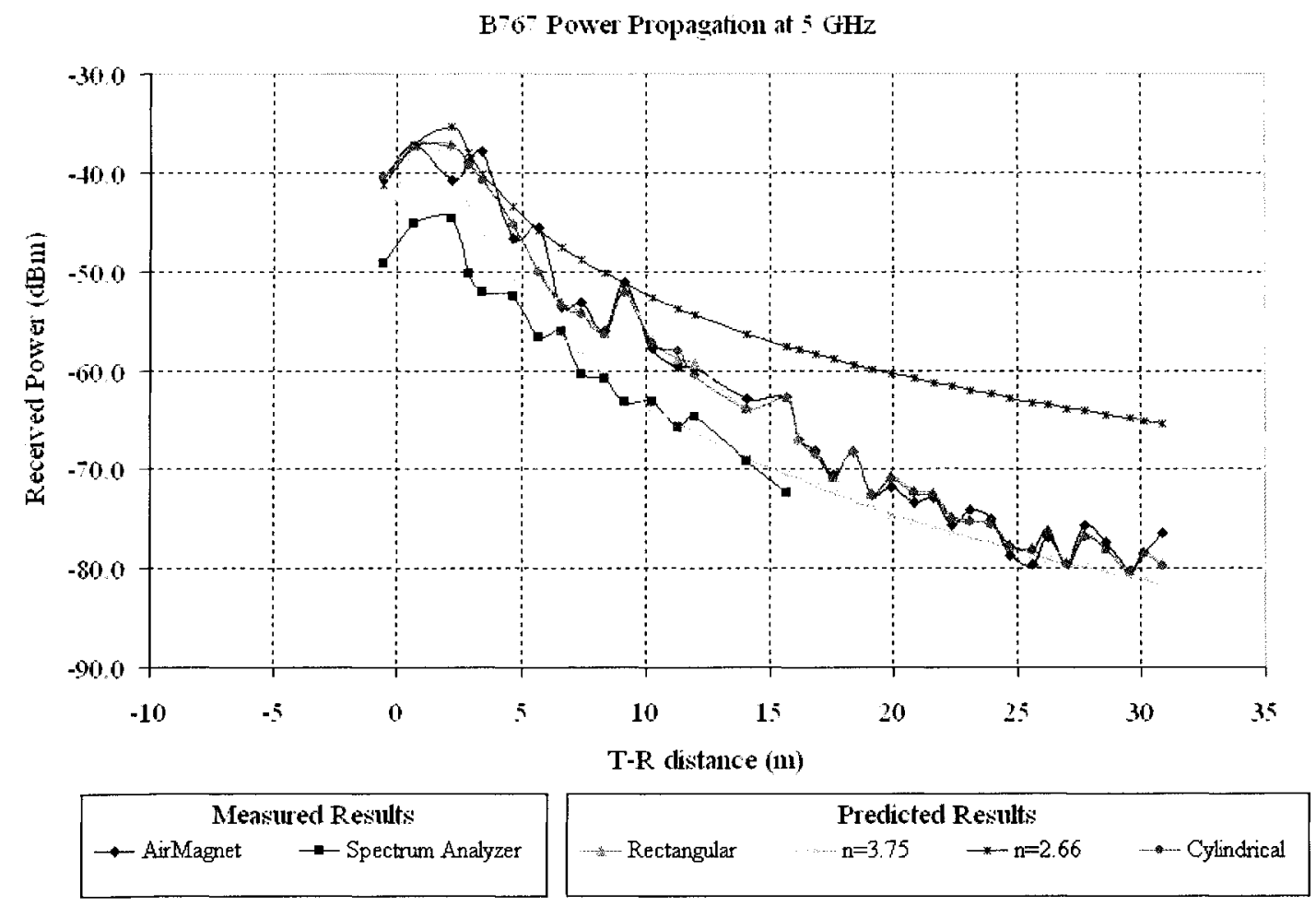

Figure 38: B767 results for 802.11a.

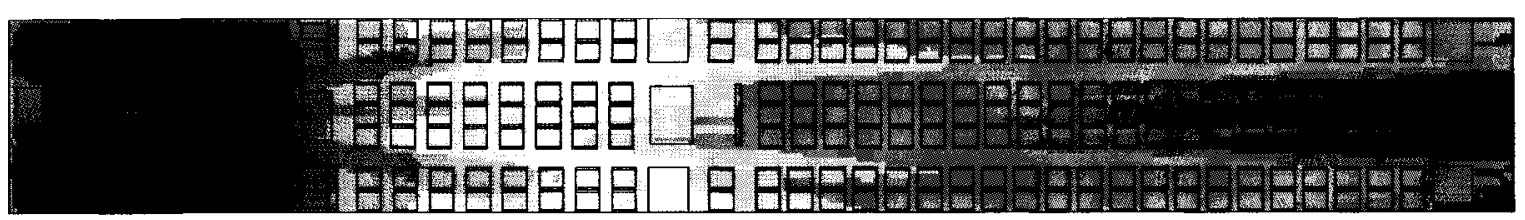

Figure 39: B767 802.11a power propagation using Grid Coverage Mode. 


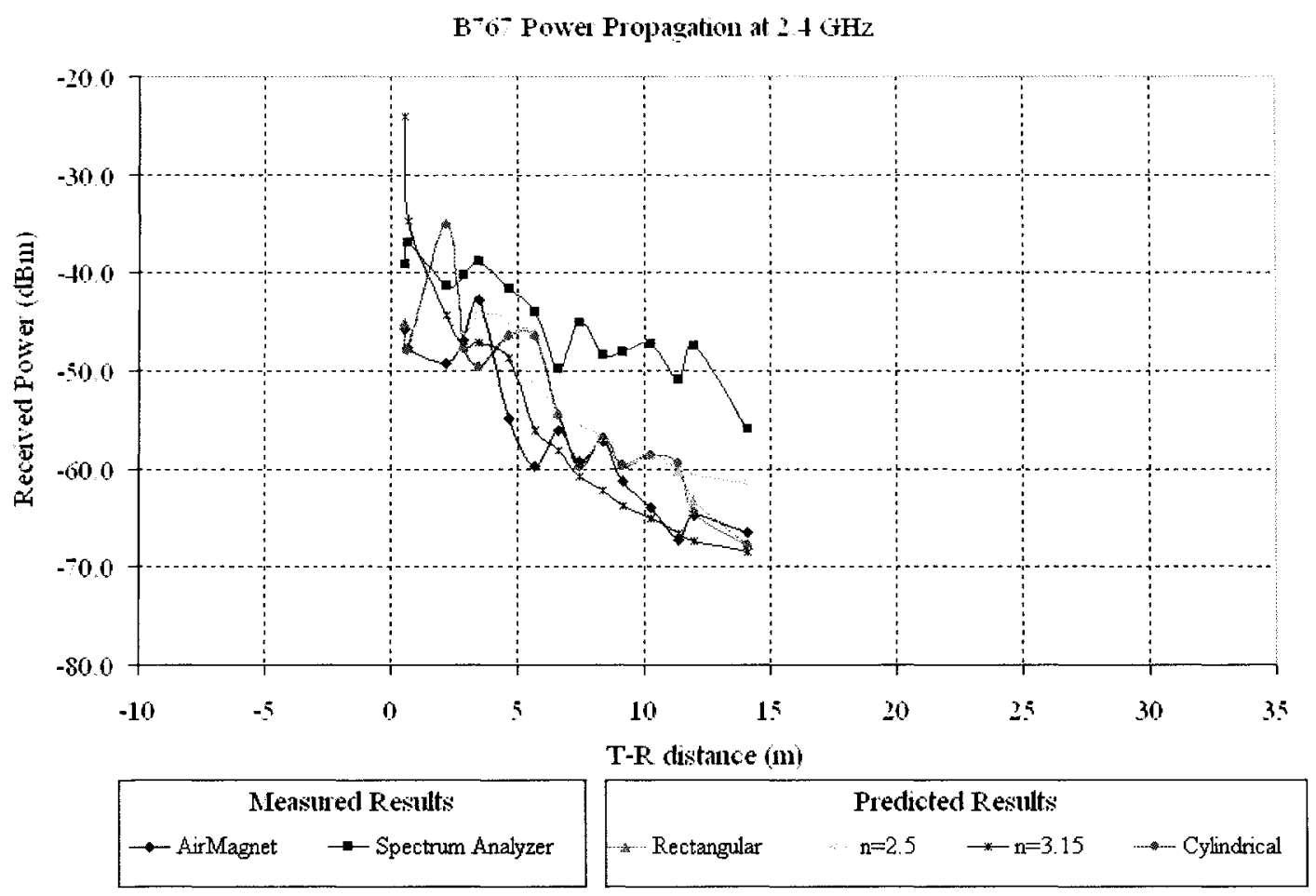

Figure 40: B767 results for 802.11b.

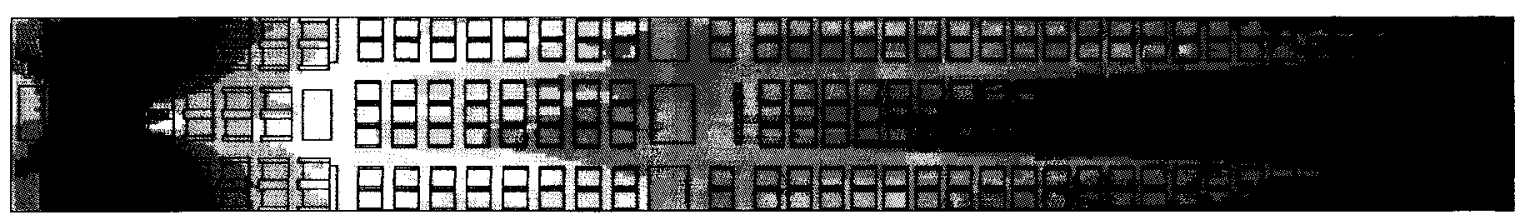

Figure 41: B767 802.11b power propagation using Grid Coverage Mode.

Table 12: Statistical analysis of $B 767$ results.

\begin{tabular}{|l|r|r|r|r|c|}
\hline & \multicolumn{2}{|c|}{ 802.11a } & \multicolumn{2}{c|}{ 802.11b } & \\
\hline Rectangular & 0.88 & 1.34 & 4.29 & 4.53 & AirMagnet \\
\hline Cylindrical & 0.93 & 1.39 & 4.20 & 6.25 & AirMagnet \\
\hline Rectangular & 7.50 & 7.87 & 8.95 & 9.68 & Spectrum Analyzer \\
\hline Cylindrical & 7.26 & 7.67 & 9.16 & 9.93 & Spectrum Analyzer \\
\hline$n_{\text {AirMagnet }}$ & 3.82 & 4.58 & 6.06 & 8.04 & AirMagnet \\
\hline$n_{\text {Spectrum Analyzer }}$ & 10.75 & 10.90 & 11.66 & 12.75 & Spectrum Analyzer \\
\hline & MAE & RMSE & MAE & RMSE & \\
\hline
\end{tabular}


B777 Results

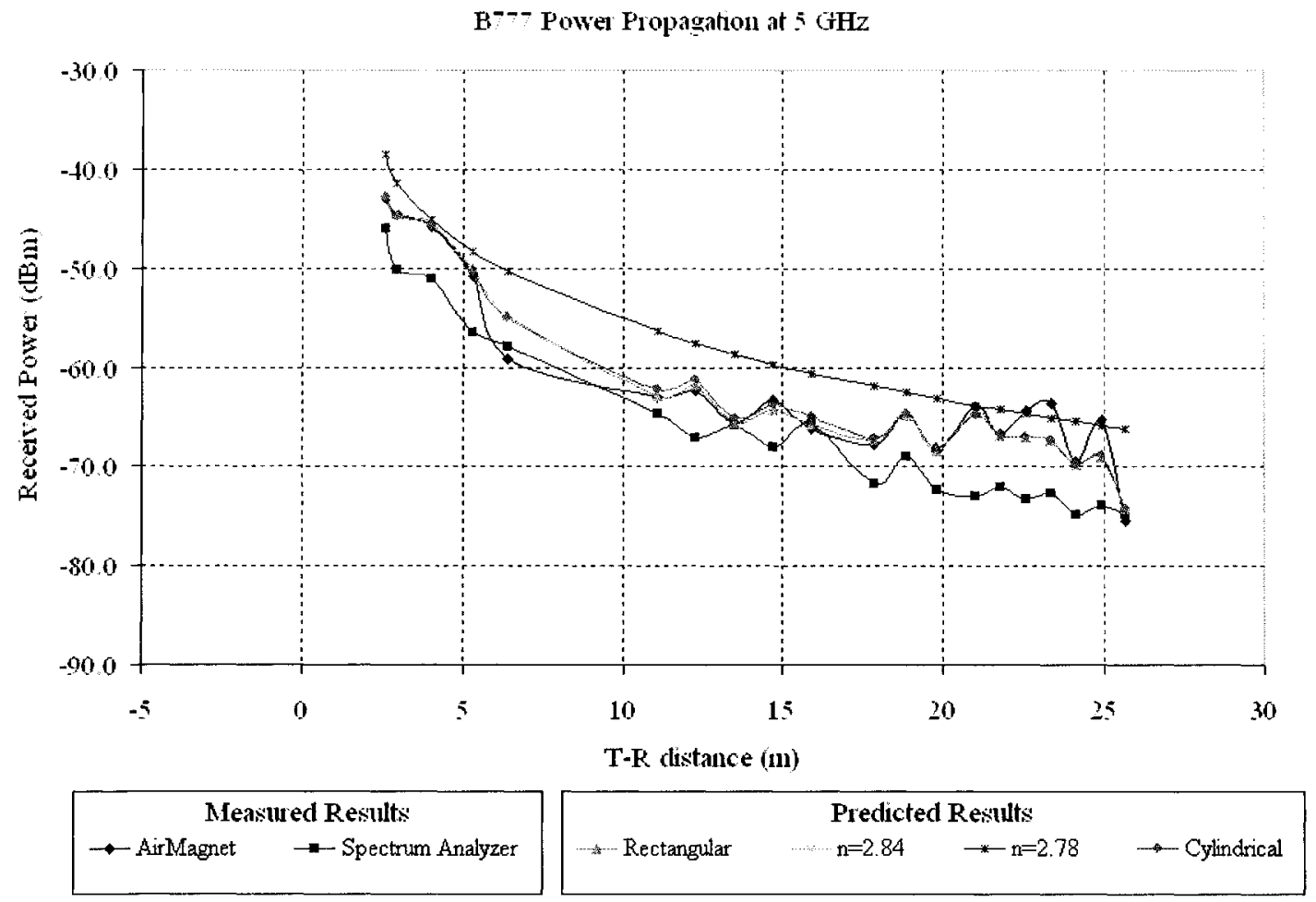

Figure 42: B777 results for 802.11a.

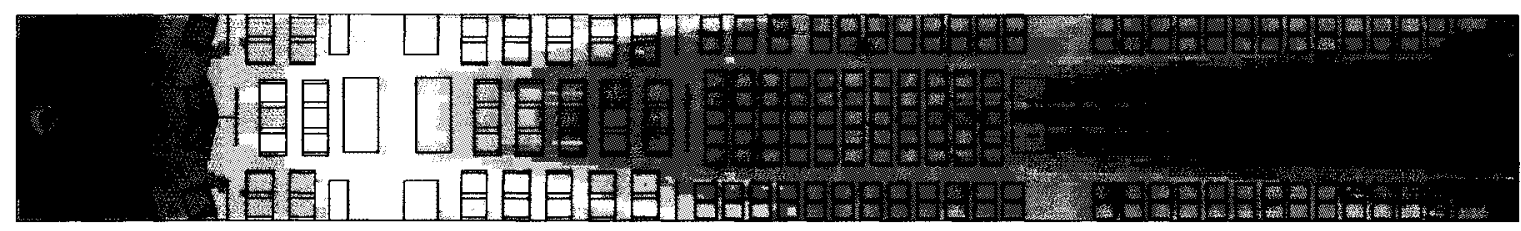

Figure 43: B777 802.11a power propagation using Grid Coverage Mode. 


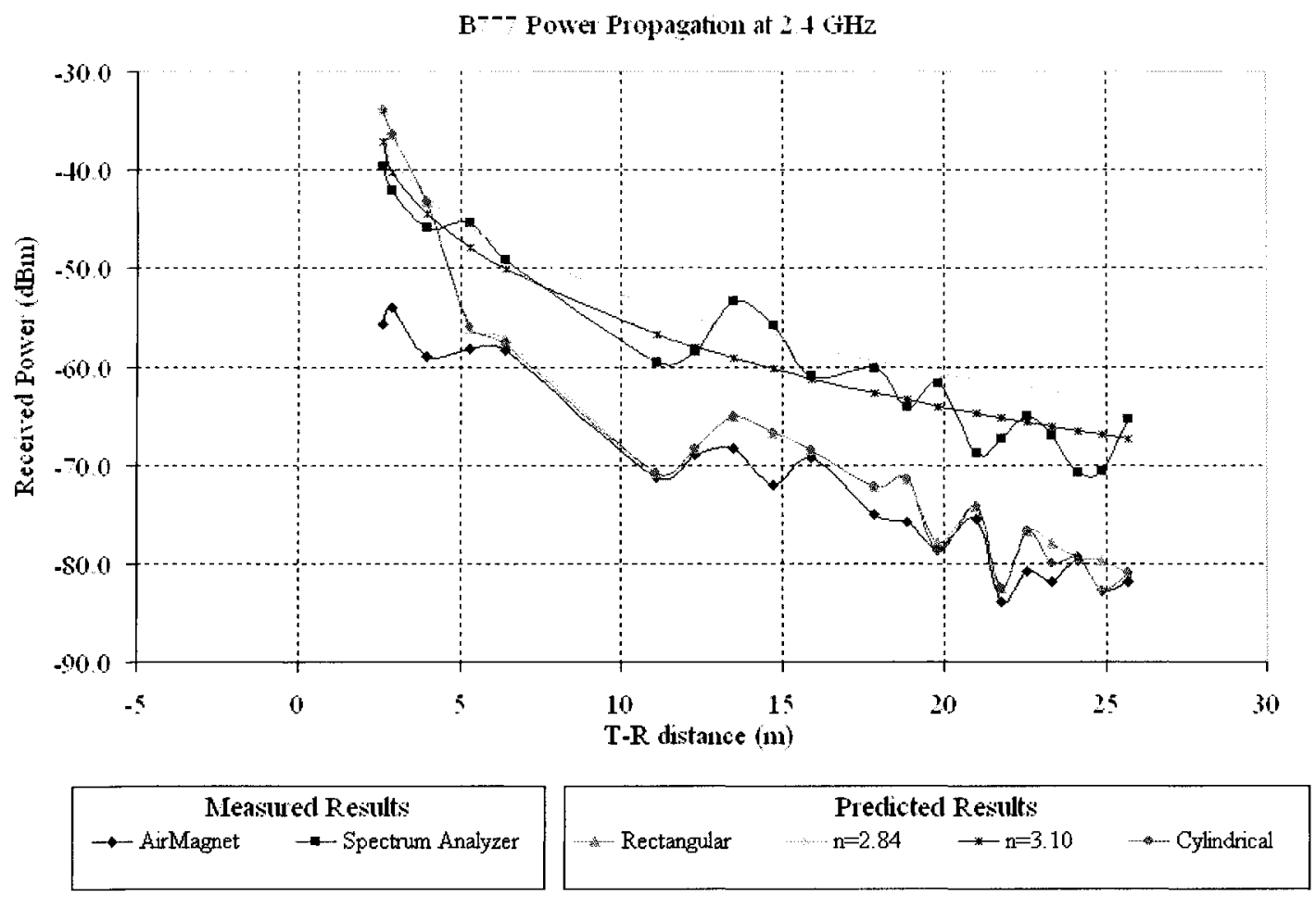

Figure 44: B777 results for $802.11 b$.

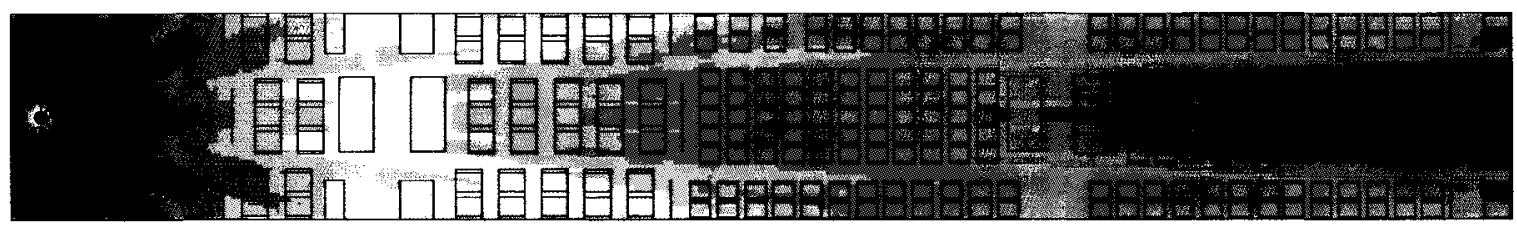

Figure 45: B777 802.11b power propagation using Grid Coverage Mode.

Table 13: Statistical analysis of $B 777$ results.

\begin{tabular}{|l|r|r|r|r|l|}
\hline & \multicolumn{2}{|c|}{$802.11 a$} & \multicolumn{2}{c|}{$802.11 b$} & \\
\hline Rectangular & 1.66 & 2.78 & 4.58 & 7.59 & AirMagnet \\
\hline Cylindrical & 1.06 & 1.60 & 4.29 & 7.52 & AirMagnet \\
\hline Rectangular & 4.28 & 4.78 & 9.78 & 10.37 & Spectrum Analyzer \\
\hline Cylindrical & 4.40 & 4.80 & 10.11 & 10.73 & Spectrum Analyzer \\
\hline $\boldsymbol{n}_{\text {AirMagnet }}$ & 4.32 & 5.10 & 16.06 & 16.34 & AirMagnet \\
\hline $\boldsymbol{n}_{\text {Spectrum Analyzer }}$ & 7.98 & 8.08 & 2.30 & 2.71 & Spectrum Analyzer \\
\hline & MAE & RMSE & MAE & RMSE & \\
\hline
\end{tabular}




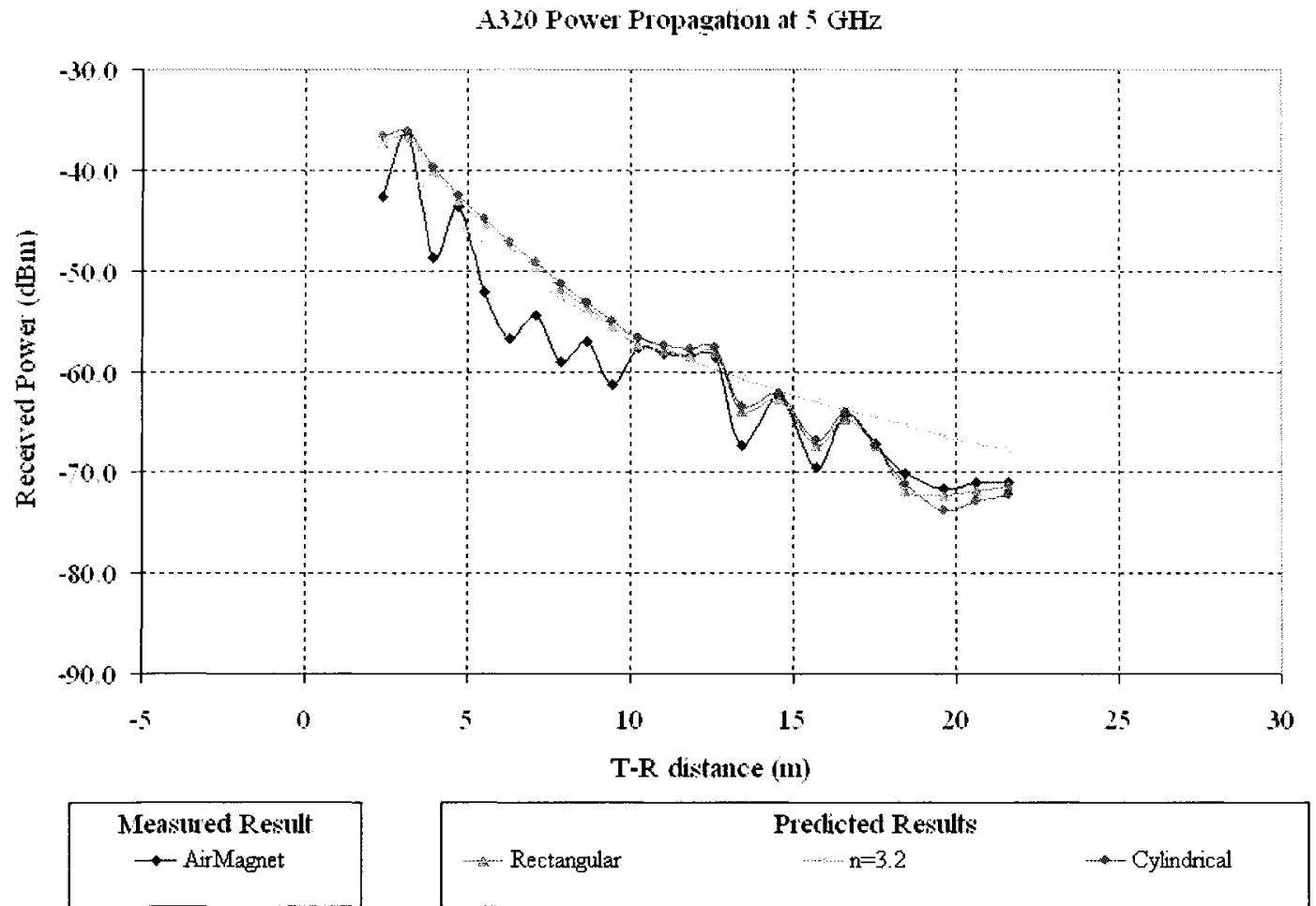

Figure 46: A320 results for 802.11a.

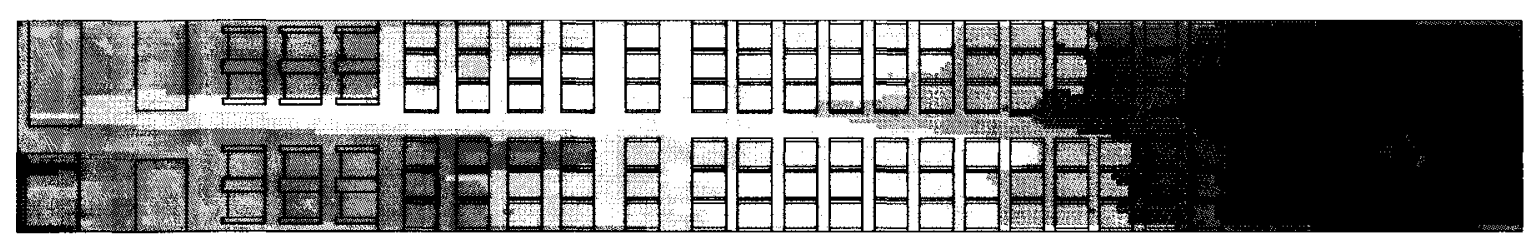

Figure 47: A320 802.11a 11b power propagation using Grid Coverage Mode. 
A320 Power Propagation at $2.4 \mathrm{GHz}$

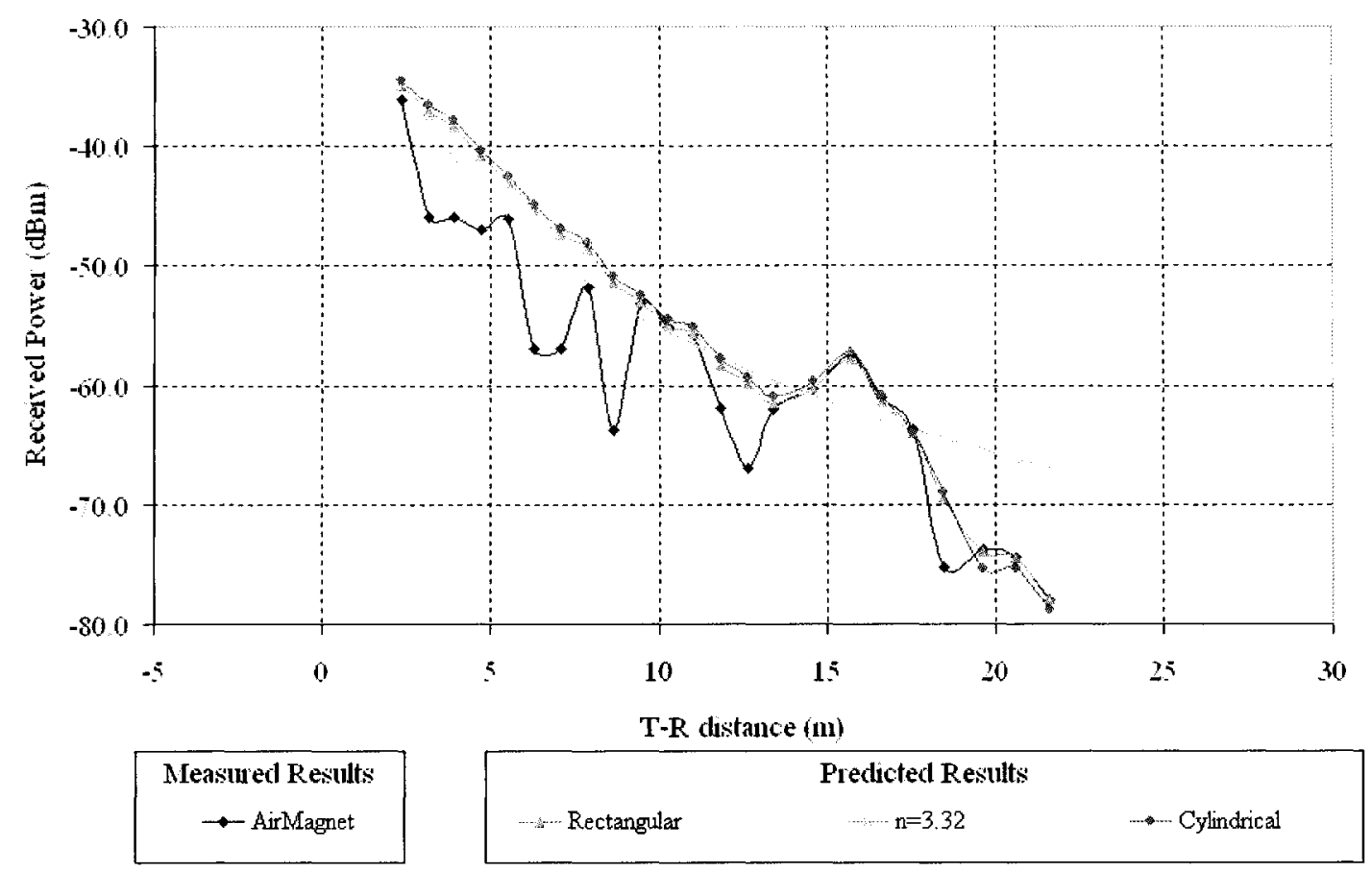

Figure 48: A320 results for $802.11 b$.

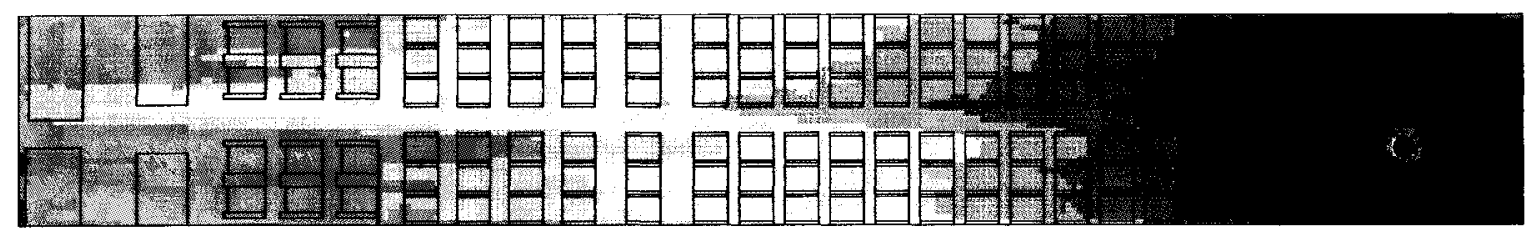

Figure 49: A320 802.11b 11b power propagation using Grid Coverage Mode.

Table 14: Statistical analysis of $\mathbf{A} 320$ results. (Spectrum Analyzer measurements were not recorded in [8])

\begin{tabular}{|l|r|r|r|r|r|}
\hline & \multicolumn{2}{|c|}{$\mathbf{8 0 2 . 1 1 a}$} & \multicolumn{2}{c|}{$\mathbf{8 0 2 . 1 1 b}$} & \\
\hline Rectangular & 3.28 & 4.92 & 3.59 & 5.41 & AirMagnet \\
\hline Rectangular & 3.17 & 4.38 & 4.08 & 5.77 & AirMagnet \\
\hline$n_{\text {AirMagnet }}$ & 3.65 & 4.28 & 4.69 & 6.07 & AirMagnet \\
\hline & MAE & RMSE & MAE & RMSE & \\
\hline
\end{tabular}




\section{Analysis}

The prediction results presented exhibit low error compared to the field measurements. The models including the interior of the planes were the most accurate, reducing the error as much as $4 \mathrm{dBm}$. However, results from the distance dependent models were still somewhat accurate and may suffice for some applications.

As expected, the results from the rectangular and cylindrical models were quite similar. In fact the observed differences are most likely due to rounding the T-R distance, $d$ (EnterprisePlanner ${ }^{\circledR}$ rounds to two digits). In all of the aircraft, the predicted measurements were most comparable to the AirMagnet measurements. As noted in Chapter II, the AirMagnet and Spectrum Analyzer measurements differed from one another as well. In some cases the two field measurements differed by more than 10 $\mathrm{dBm}$. Additionally, the predicted results were also more accurate at predicting $802.11 \mathrm{a}$ transmission. This could be attributed to the type of antenna assigned to the AP (the antenna pattern for 802.11a was incorporated into its design). Further investigation into the antenna pattern and gain of this system may be necessary. 


\section{CHAPTER VII}

\section{CONCLUSIONS}

The purpose of this thesis was to determine if EnterprisePlanner ${ }^{\circledR}$, a commercially available software application, could be used to model the EME inside aircraft cabins. The software was evaluated by comparing the predicted results with field measurements taken on several aircraft. The field measurements taken from [8] were presented and some additional analysis was provided. Two representations for each aircraft were developed and analyzed with several different prediction modes within EnterprisePlanner ${ }^{\circledR}$. The results and analysis of these models were presented in Chapter VI.

The statistical analysis and graphical comparisons from Chapter VI demonstrate that EnterprisePlanner ${ }^{\circledR}$ provides accurate results. Furthermore, including the internal components of the aircraft in the predictions significantly reduced the associated error. In addition, the results presented show that including internal components yields an approximate curve to that of the simulated data. The nature of RF measurements suggests that an approximation of the overall shape of the measured curve may lead to a more accurate simulation rather than relying on statistical analysis of specific values (since these values are likely to change from test to test). In this respect, as shown in Chapter VI, rectangular or cylindrical model is preferred over the distance dependent model.

EnterprisePlanner ${ }^{\circledR}$ can be utilized for designing and planning future wireless networks to be implemented onboard commercial aircraft. This will reduce the cost associated with field measurements and the aircrafts time out of service. Additionally, EnterprisePlanner ${ }^{\circledR}$ is capable of predicting other types of wireless systems including cellular systems. These features make it a valuable tool for researchers and the aviation industry. 


\section{Proposed Future Work}

The latest version of EnterprisePlanner ${ }^{\circledR}$ includes a ray tracing prediction model. Future work could study the accuracy of this model compared to the simplistic path loss models used for this thesis. Although the models presented provide statistically similar results to field measurements, the ray tracing method may give further insight to the EME within the cabin and supplement the path loss models.

Additionally, many of the approximations made throughout this thesis could be verified or measured. This includes general dimensions of the aircraft and its components, EM characteristics of the AP and client card, and material attenuation factors. The absorption properties of the internal components are of particular interest and could be calculated by taking additional field measurements. 


\section{REFERENCES}

[1] “Wireless Solutions," http://www.portal.com/solutions/wireless/, June 10, 2005.

[2] S. Lawson, "In-Flight Cell Phone Systems Gain Altitude," PC World, June 15, 2005, http://www.pcworld.com/news/article/0, aid,121399,00.asp, June 20, 2005.

[3] J. Blau, "In-Flight Broadband Ready for Takeoff," PC World, May 27, 2003, http://www.pcworld.com/news/article/0,aid,110893,00.asp, July 12, 2004.

[4] M.T.P. Salud, "Investigation of RF Emissions From Wireless Networks as a Threat to Avionic Systems," NASA Langley Research Center, Technical Report CR-2002-211941, October 2002.

[5] T.X. Nguyen, S.V. Koppen, J.J. Ely, R.A. Williams, M.T.P. Salud and L. J. Smith, "Portable Wireless LAN Device and Two-Way Radio Threat Assessment for Aircraft Navigation Radios," NASA Langley Research Center, Technical Report TP-2003-212438, July 2003.

[6] J. Blau, "Scandinavian Airlines Makes a Connexion," PC World, July 03, 2003, http://www.pcworld.com/news/article/0,aid,111446,00.asp, July 12, 2004.

[7] J.W. Ramsey, "Wireless Undeterred," Avionics Magazine. February 2004. http://avionicsmagazine.com/cgi/av/show_mag.cgi?pub=av\&mon=0204, March 12, 2004.

[8] F. Whetten, A. Soroker, and D. Whetten, "802.11 Wireless Network Performances within Aircraft Cabins," Embry-Riddle Aeronautical Univ., Technical Report, June 25, 2003.

[9] A. Taflove and S.C. Hagness, Computation Electrodynamics: The FiniteDifference Time-Domain Method, $2^{\text {nd }}$ ed., Artech House, Inc., 2000.

[10] A.F. Peterson, S.L. Ray and R. Mittra. Computational Methods for Electromagnetics. IEEE Press, 1998.

[11] S.Y. Seidel, and T.S. Rappaport, "Site-Specific Propagation Prediction for Wireless In-Building Personal Communication System Design," IEEE Trans. Veh. Technol., Vol. 43, no. 8, pp. 879-891, Nov. 1994.

[12] L.V. Blake, Antennas. New York: John Wiley \& Sons, 1966.

[13] S.A. Schelkunoff and H.T. Friis, Antennas: Theory and Practice, New York: John Wiley \& Sons, 1952.

[14] G. Durgin and T.S. Rappaport, "Measurements and Models for Radio Path Loss and Penetration Loss In and Around Homes and Trees at $5.85 \mathrm{GHz}$," IEEE Trans. Commun., vol. 46, no. 11, pp. 1584-1496, Nov. 1998.

[15] Y.P. Zhang. "Indoor Radiated-Mode Leaky Feeder Propagation at $2.0 \mathrm{GHz}$," IEEE Trans. Veh. Technol., vol. 50, no. 2, pp. 536-545, Mar. 2001. 
[16] N. R. Diaz, J.E. J. Esquitino, O. Franzrahe, T. Eisenberg, P. Veith, and A. Pohl. "In-Cabin Channel Measurement Performance and Result Report," Wireless Cabin, Information Society Technologies, Technical Report IST-2001-37466, 2001.

[17] D. Dobkin, "Indoor Propagation and Wavelength," WJ Communications, RF 802.11 Propagation, Technical Report, July 10, 2001.

[18] Jackson. Classical Electrodynamics, $2^{\text {nd }}$ ed., New York: John Wiley \& Sons, 1974.

[19] J. Unger, Deploying License-Free Wireless Wide-Area Networks. Cisco Press, 2003.

[20] D. Cheung and C. Prettie, "A Path Loss Comparison Between the $5 \mathrm{GHz}$ UNII Band (802.11a) and the 2.4 GHz ISM Band (802.11b)," Intel Labs Intel Inc., Technical Report, January 2002.

[21] "Comparing Performance of 802.11b and802.11a Wireless Technologies," 3Com Corp., Technical Report, 2001.

[22] J.C. Chen, J.M. Gilbert, "Measured Performance of 5-GHz 802.11a Wireless LAN Systems" Atheros Communications, Inc., Technical Report, August 2001.

[23] J. Zyren, E. Enders, and T. Edmondson, CommsDesign.com, January 14, 2003, "802.11g Starts Answering WLAN Range Questions," http://www.commsdesign.com/showArticle.jhtml?articleID $=16500198$, December $10,2004$.

[24] EnterprisePlanner 9.0 User's Manual, Wireless Valley, Inc., 2004.

[25] T.S. Rappaport, Wireless Communications: Principles and Practice, Prentice Hall, 1996.

[26] Homepage, Boeing, Inc., www.boeing.com, June 6, 2005.

[27] G. Hankins, J.H. Beggs, and L. Vahala. "Electromagnetic Propagation Prediction inside Aircraft Cabins" in IEEE Antennas and Propagation Symposium, Monterey, CA June 2004, pp. 2227-2230.

[28] Homepage, Airbus, Inc., www.airbus.com, June 6, 2005. 


\section{VITA}

\section{GENEVIEVE HANKINS}

Department of Electrical and Computer Engineering

Old Dominion University

Norfolk, VA 23529

(757) $683-3741$
4940 Barn Swallow Drive

Chesapeake, VA 23321

ghankins@gmail.com

(757) 488-3148

\section{EDUCATION}

Master of Science in Electrical Engineering

August 2005

Old Dominion University Norfolk, Virginia

Bachelor of Science in Electrical Engineering Minor in Mathematics

May 2004

Old Dominion University Norfolk, Virginia

\section{EXPERIENCE}

May 2003-Present

Graduate Student Researcher

March 2005-May 2005

Graduate Student Teacher

January 2004-May 2004

Teacher's Assistant
NASA Langley Research Center

Hampton, VA

Old Dominion University Norfolk, Virginia

Old Dominion University Norfolk, Virginia

\section{PUBLICATIONS}

G. Hankins, J.H. Beggs, and L. Vahala. "Electromagnetic Propagation Prediction inside Aircraft Cabins" in IEEE Antennas and Propagation International. Symposium, Monterey, CA June 2004.

G. Hankins, J.H. Beggs, and L. Vahala. "802.1 lab Propagation Prediction inside a B777" in IEEE/ACES International Conference on Wireless Communication and Applied Computational Electromagnetics, Honolulu, HI April 2005.

G. Hankins, J.H. Beggs, and L. Vahala. "Propagation Prediction inside a B767 in the $2.4 \mathrm{GHz}$ and $5 \mathrm{GHz}$ Radio Bands" in IEEE Antennas and Propagation International Symposium, Washington, D.C. July 2005.

\section{ASSOCIATION MEMBERSHIPS}

- Institute of Electrical and Electronics Engineers (IEEE) since 2002

- Society of Women Engineers (SWE), 2003

\section{HONORS AND AWARDS}

- One of three hundred nationwide selected for the NASA Graduate Student Researcher's Program, 2004-2005

- Student member of the Dean's Advisory Board, 2003-2005

- Service Award, Old Dominion University Department of Electrical and Computer Engineering, May 2004

- $\quad 1^{\text {st }}$ place, Old Dominion University Department of Electrical and Computer Engineering Student Paper Contest, May 2004

- $3^{\text {rd }}$ place, IEEE Region 3 Student Paper Contest, March 2004

- $\quad 1^{\text {st }}$ place, IEEE Hampton Roads Section Student Paper Contest, January 2004

- Recipient of the Governor's Technology Scholarship for undergraduate studies 